PHYSICA SCRIPTA

(The Royal Swedish Academy of Sciences)

Online ISSN: 1402-4896; Print ISSN: 0031-8949; Impact factor = 1.985

Publisher- Institute of Physics (IOP)

https://iopscience.iop.org/journal/1402-4896

Accepted October $27^{\text {th }} 2020$

\title{
Finite difference computation of free magneto-convective Powell-Eyring nanofluid flow over a permeable cylinder with variable thermal conductivity
}

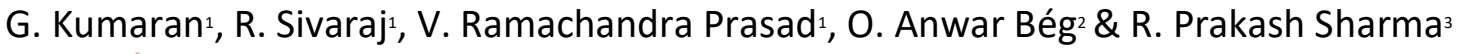 \\ ${ }^{1}$ Department of Mathematics, School of Advanced Sciences, \\ Vellore Institute of Technology, Vellore 632014, India \\ ${ }^{2}$ Multi-Physical Engineering Sciences Group (MPESG), Aeronautical and Mechanical \\ Engineering Department, School of Science, Engineering and Environment, \\ University of Salford,Manchester M54WT, UK \\ ${ }^{3}$ Department of Mechanical Engineering, NIT Arunachal Pradesh, Yupia, Papum \\ Pare,Arunachal Pradesh-791112, India. \\ E-mail: gkumaranmsc31@gmail.com, ${ }^{*}$ sivaraj.kpm@gmail.com, \\ rcpmaths@gmail.com, gortoab@gmail.com, rpsharma@nitap.ac.in
}

\begin{abstract}
It is essential to account the variability in thermophysical properties such as thermal conductivity to obtain the characteristics of transport properties in industrial thermal systems more accurately. This phenomenon is especially significant in coating protection for rocket chambers, heat exchangers and power generation, wherein cooling techniques are required for sustaining temperature regulation and structural material integrity. At high operating temperatures, the working fluid and hot walls generally emit appreciable radiation. Mathematical models are therefore required which simultaneously analyse all three modes of heat transfer in addition to viscous flow and a variety of other effects including reactions (corrosion, combustion), mass diffusion and rheological behaviour. The modern thrust in nanoscale materials is a major consideration. Motivated by these applications, in this paper, a theoretical examination is implemented to analyse the impact of thermal conductivity variation and thermal radiation on chemically reacting, free convective Powell-Eyring nanofluid flow over a cylinder. The nanoscale effects are accounted by employing the Buongiorno model. The transformed governing equations are numerically solved by using Keller box method under suitable boundary conditions. The comparison results reveal that the obtained results find an excellent match with the results in the literature. The graphs and tables elucidate the impacts of various pertinent parameters on thermosolutal transport characteristics. It is to be noted that amplifying thermal conductivity variation rises fluid velocity and temperature. Velocity of the fluid decelerates for elevating Darcy number. Magnifying the radiation corresponds to weak radiative flux and stronger thermal conduction which decrease the heat transfer whereas the mass transfer is increased. Furthermore, nanoparticle concentration decreases with greater first order chemical reaction and Brownian motion parameter values.
\end{abstract}

Keywords: Powell-Eyring nanofluid, variable thermal conductivity, chemical reaction, thermal radiation, Keller box method. 


\section{Nomenclature}

a cylinder radius

$u, v \quad$ velocity components along the $x$ and $y$ directions

$B_{0} \quad$ strength of constant magnetic field

$g_{a}$ gravitational acceleration

$k_{f} \quad$ variable thermal conductivity of the fluid

$k_{0} \quad$ ambient thermal conductivity of the fluid

$T$ fluid temperature

$T_{w} \quad$ temperature of the cylindrical surface

$T_{\infty}$ ambient temperature

C fluid concentration

$C_{w}$ concentration of the cylindrical surface

$C_{\infty}$ ambient concentration

$k_{a} \quad$ mean absorption coefficient

Gr Grashof number

$f_{w} \quad$ blowing/suction parameter

Ha magnetic parameter

Da Darcy parameter

$P_{r} \quad$ Prandtl number

$N_{t}$ thermophoresis parameter

$N_{b} \quad$ Brownian motion parameter

$R$ thermal radiation parameter

Sc Schmidt number

$K r$ chemical reaction parameter

$N$ buoyancy ratio parameter 


\section{Greeek symbols}

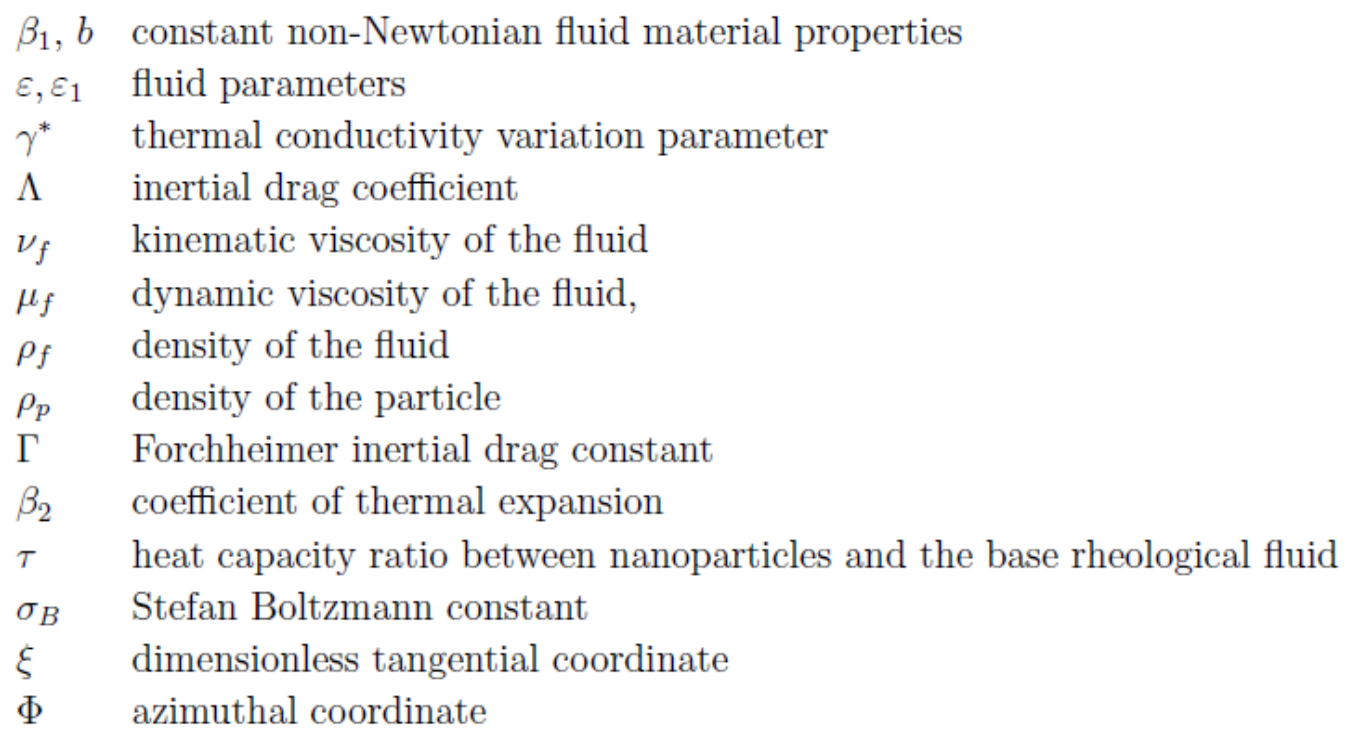

\section{Introduction}

The transport properties of Non-Newtonian fluids continue to attract the attention of many researchers due to numerous applications in hypergolic rocket fuels (gel propellants), food preservation techniques, chemical processing, energy production, etc. Shampoo, paper pulp, oils, paints, coating melts and liquid polymers exemplify the rheological behaviours of non-Newtonian liquids. In 1944, Powell and Eyring [1] introduced a pioneering non-Newtonian fluid model known as Powell-Eyring fluid. This model is a sub-class of viscoelastic fluid models and the kinetic theory based constitutive equation are formulated for this fluid. Expressing the rheology of non-Newtonian fluids at lesser shear rate and greater shear rate is quite accurately achieved in Powell- Eyring fluid model. A number of industrial smart polymers feature electrically conducting properties and these require viscous magnetohydrodynamic (MHD) models. Several investigations regarding both electro conductive and non-conductive flows of Powell-Eyring fluids have therefore appeared in the past few decades. In high temperature coating applications, radiative heat transfer plays a significant role. Motivated by this, Hayat et al. [2] deliberated the impact of variable temperature and radiative heat flux on magnetohydrodynamic Powell-Eyring nanofluid flow over a stretching cylinder and noted that an increment in radiative heat flux augments fluid temperature. Rehman et al. [3] scrutinized the transport properties of mixed convective, magnetohydrodynamic flow of Powell-Eyring fluid over an inclined cylinder and perceived that angle of inclination and magnetic field parameters notably decelerate velocity of the fluid. The impact of radiative heat flux and ohmic heating on transport properties of magnetohydrodynamic squeezing flow of Eyring-Powell fluid between two parallel plates is analyzed by Ghadikolaei et al. [4] and reported that amplifying the magnetic field parameter strengthening 
the Lorentz force which induces a decline in the fluid velocity at the bottom of the channel whereas opposite nature is seen at the top of the channel. Balazadeh et al. [5] adopted differential transformation method to analyse the characteristics of magnetohydrodynamic Eyring-Powell fluid squeezing flow in a channel and detected that fluid temperature enhances for augmenting the magnetic field parameter. Hayat et al. [6] numerically obtained a solution to characterise the Powell-Eyring nanofluid peristaltic transport with endoscopic effect, chemical reaction, heat source/sink, and viscous dissipation and observed that fluid temperature is boosted with higher heat source/sink parameter values. Salawu et al. [7] theoretically scrutinized the impact of variable thermal conductivity on chemically reactive flow of MHD Powell-Eyring fluid between parallel plates and detected that fluid temperature declines for amplifying thermal conductivity variation.

The fluid thermal conductivity is one of the significant thermo physical fluid properties which may be influenced by heat generation due to internal friction. The fluid temperature features with variable thermal conductivity enable better predictions of thermo fluid behaviour in several applications. In general, accounting the thermal conductivity variations is essential when heat transfer variation is notable. Seddeek et al. [8] utilized the Chebyshev finite difference method to investigate the impact of thermal conductivity variation on fluid transport properties over a moving surface and noticed that heat and mass transfer profiles rise for larger thermal conductivity variations. Khan and Malik [9] scrutinized the Sisko nanofluid flow over a stretching cylinder by considering the effect of thermal conductivity variations and nanoparticle flux conditions and inferred that accounting the thermal conductivity variation increases the fluid temperature. In addition, it is mentioned that the temperature variation of the liquid metals almost linearly varies with thermal conductivity variation. Sivaraj et al. [10] adopted a numerical method to analyse varying properties of the fluid on chemically reacting, free convective flow and detected that magnifying variable thermal conductivity parameter uplifts the fluid heat transfer. The absorbing-emitting fluids temperature highly depends on thermal radiation and heat transfer in such fluids due to free convection can be dominated by the heat transfer due to thermal radiation. The human body temperature is regulated on par with the surrounding temperature due to the blood flow. In several medical treatments (myalgia, muscle spasm, permanent shortening of muscle and chronic widespread pain), controlling the temperature of the blood flow is essential which may be attained with the aid of radiative heat flux. Further, role of thermal radiation is essential in many thermal therapeutic procedures [11-14]. Further, thermal radiation in viscous flow from an extending/contracting surface has a critical role in fuel cells, coating systems, shrink fit processes in packaging, liquid metal fabrication, etc. Abbas et al. [15] studied chemically reacting non-Newtonian fluid stagnation point flow with the impact of thermal radiation and observed that heat transfer reduces for magnifying the radiative heat flux. Rahman et al. [16] numerically examined the significance of radiative heat flux in the problem of non-Newtonian fluid mixed convective flow over an 
extending sheet and noticed that amplifying the radiative heat flux diminishes profiles of heat transfer rate. Sheikholeslami et al. [17] applied the homotopy analysis method to study the hydromagnetic flow in a channel with radiative heat flux and inferred that higher radiation parameter values motivate the heat transfer in the channel. Khan et al. [18] scrutinized the radiative heat flux impression on flow of non-Newtonian fluid over an extending surface by employing Runge-Kutta Fehlberg quadrature and detected that heat distribution enhances for magnifying radiative heat flux.

Thermophoretic motion i.e. the translocation of suspended particles in a fluid under applied thermal gradients, has been applied in many different industrial technologies including commercial electrostatic precipitators, optical fibers, fouling, etc. This phenomenon has been identified as a key mechanism for thermal enhancement in the new generation of nanoscale-engineering liquids known as nanofluids. Thermophoresis features in isolating different polymer particles in the stream field of fractionation. In nanofluids, the Buongiorno model provided a good framework for boundary layer analysis and inferred that both thermophoresis and Brownian motion are important slip mechanisms to analyse the transport properties of nanofluids. A number of recent studies have therefore considered this formulation. Sandeep et al. [19] scrutinized magnetohydrodynamic, non-Newtonian fluid convective flow over a porous surface by accounting suction/injection and concluded that magnification in thermophoresis accentuates rate of heat and rate mass transfer profiles. Makinde and Animasaun [20] investigated the magnetohydrodynamic boundary layer flow from a paraboloid of revolution by accounting chemical reaction and Brownian motion using a numerical scheme and found that heat transfer is enhanced for increasing the thermophoresis parameter. Hamid et al. [21] numerically computed the unsteady magnetohydrodynamic Williamson nanofluid chemically reacting flow over a cylinder by considering activation energy. Mittal and $\mathrm{Pa}-$ tel [22] obtained homotopy solutions for magnetohydrodynamic Casson nanofluid mixed convective flow with heat source and noted that fluid temperature improves for magnifying thermophoresis parameter. Boundary layer flows from an extending surface arise in the extrusion of elastic sheets, plastic films, fiber spinning, thinning of copper wires, hot rolling, etc. Hussain and Ullah [23] studied magnetic boundary layer convection with variable fluid viscosity (using the Reynolds and Vogel exponential models) for a short memory viscoelastic liquid from a stretching cylinder. Tamoor [24] explored the influence of suction/blowing on magnetohydrodynamic viscous fluid flow over a cylindrical surface by employing shooting techniques and reported that augmentations in suction parameter decrease fluid flow and heat transfer.

An inspection of the literature has revealed that many computational studies of external boundary layer flows of rheological nanofluids have featured ordinary differential equation boundary value problems i.e. only a single space variable has been considered. Furthermore, most of the studies have ignored thermal conductivity variation. The objective of the current work is therefore to present a more generalized two-dimensional ap- 
proach to axisymmetric magnetohydrodynamic chemically reactive rheological nanofluid boundary layer flow over a porous cylindrical body with radiative heat flux. The regime is immersed in a high permeability porous medium and both linear and quadratic drag forces are considered via the robust Darcy-Forchheimer model. Suction and injection are simulated at the cylindrical surface. Thermal radiation is approximated based on Rosselands diffusion flux model. The Powell-Eyring non-Newtonian and Buongiorno nanoscale models are deployed. The considered homogenous destructive type chemical reaction is of first order. The steady-state conservation equations are transformed, rendered non-dimensional and then solved with appropriate wall and free stream boundary conditions by utilising the unconditionally stable, Keller box method. The present study may be useful in applications pertaining to intelligent coatings for cylindrical engineering components (e.g. photovoltaic cylindrical solar collectors and pipelines), surficial protection of autonomous robotic limbs with magnetic polymers, etc. Several pertinent parameters impact on fluid transport properties are demonstrated through tables and graphs. Verification of the accurateness of present solution with earlier published works is included. The present work constitutes a significant extension to the literature on the numerical simulation of enrobing flows of curved bodies with magnetic nanomaterials.

\section{Mathematical Formulation}

A two-dimensional, steady, laminar, non-Darcy flow of an incompressible, electrically conducting Powell-Eyring nanofluid external to a horizontal cylinder embedded in a permeable regime is considered. The flow geometry of the problem is elucidated in Fig.1. A uniform magnetic field with constant strength is enforced in transverse to the enrobing boundary layer flow. The radiative heat flux and first order chemical reaction are accounted. The temperature and concentration of the cylindrical surface are in order higher than the ambient temperature $\left(T_{w}>T_{\infty}\right)$ and ambient concentration $\left(C_{w}>C_{\infty}\right)$. Radiative flux $q_{r}$ acts in the radial direction. A dilute rheological nanofluid is considered where the nanoparticles are in local thermal equilibrium.

The cylinder surface is electrically insulated and polarization voltage is neglected. The non-Newtonian Powell-Eyring constitutive equation is expressed as follows [25,26]:

$$
\tau_{i j}=\frac{1}{\beta_{1}} \sinh ^{-1}\left(\frac{1}{b} \frac{\partial u}{\partial x}\right)+\mu_{f} \frac{\partial u}{\partial x} .
$$

The stress tensor in Eqn. (1) can be simplified by assuming:

$$
\sinh ^{-1}\left(\frac{1}{b} \frac{\partial u}{\partial x}\right) \cong \frac{1}{b} \frac{\partial u}{\partial x}-\frac{1}{6}\left(\frac{1}{b} \frac{\partial u}{\partial x}\right)^{3}, \quad\left|\frac{1}{b} \frac{\partial u}{\partial x}\right|<1
$$

The governing equations for steady thermo solutal MHD Powell-Eyring nanofluid with variable thermal conductivity and Darcy-Forchheimer porous media drag forces are expressed by generalizing the models in [27, 28, 59-61] to give:

$$
\frac{\partial u}{\partial x}+\frac{\partial v}{\partial y}=0
$$




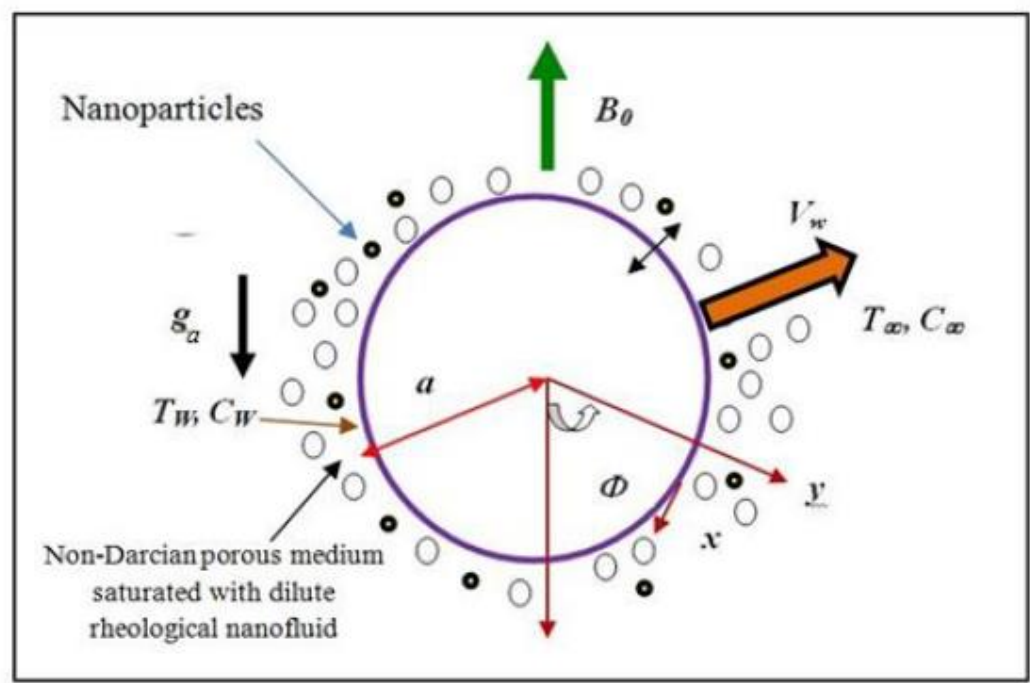

Figure 1. Flow geometry of the problem

$$
\begin{gathered}
u \frac{\partial u}{\partial x}+v \frac{\partial u}{\partial y}=\left[\nu_{f}+\frac{1}{b \beta_{1}}\right] \frac{\partial^{2} u}{\partial y^{2}}-\frac{1}{2 b^{3} \beta_{1}}\left(\frac{\partial u}{\partial y}\right)^{2} \frac{\partial^{2} u}{\partial y^{2}}-\Gamma u^{2} \\
-\left(\frac{\sigma_{f} \beta_{0}^{2}}{\rho_{f}}+\frac{\nu_{f}}{K}\right) u+g_{a}\left[\begin{array}{c}
\beta_{2}\left(1-C_{\infty}\right) \rho_{f}\left(T-T_{\infty}\right) \\
-\frac{1}{\rho_{f}}\left(\rho_{p}-\rho_{f}\right)\left(C-C_{\infty}\right)
\end{array}\right] \sin \left(\frac{x}{a}\right) \\
u \frac{\partial T}{\partial x}+v \frac{\partial T}{\partial y}=\frac{1}{\rho_{f}\left(C_{p}\right)_{f}} \frac{\partial}{\partial y}\left(k_{f}(T) \frac{\partial T}{\partial y}\right)-\frac{1}{\rho_{f}\left(C_{p}\right)_{f}} \frac{\partial q_{r}}{\partial y} \\
+\tau\left[D_{B} \frac{\partial C}{\partial y} \frac{\partial T}{\partial y}+\frac{D_{T}}{T_{\infty}}\left(\frac{\partial T}{\partial y}\right)^{2}\right], \\
u \frac{\partial C}{\partial x}+v \frac{\partial C}{\partial y}=D_{B} \frac{\partial^{2} C}{\partial y^{2}}+\frac{D_{T}}{T_{\infty}} \frac{\partial^{2} T}{\partial y^{2}}-K_{c}\left(C-C_{\infty}\right),
\end{gathered}
$$

The boundary conditions for the problem are as follows

$$
\begin{aligned}
& y=0: u=0, v=V_{w}, T=T_{w}, C=C_{w} \\
& y \rightarrow \infty: u \rightarrow 0, T \rightarrow T_{\infty}, C \rightarrow C_{\infty} .
\end{aligned}
$$

The stream function $\psi$ is considered as $u=\partial \psi / \partial y$ and $v=-\partial \psi / \partial x$ to satisfy the continuity equation. The following dimensionless variables are invoked:

$$
\begin{aligned}
& \xi=\frac{x}{a}, \eta=\frac{y}{a} \sqrt[4]{G r}, f(\xi, \eta)=\frac{\psi}{\nu_{f} \xi \sqrt[4]{G r}} \\
& \theta(\xi, \eta)=\frac{T-T_{\infty}}{T_{w}-T_{\infty}}, \phi(\xi, \eta)=\frac{C-C_{\infty}}{C_{w}-C_{\infty}} .
\end{aligned}
$$

Based on Rosseland model [29], the radiative heat flux $\left(q_{r}\right)$ is modelled as:

$$
q_{r}=-\frac{4}{3} \frac{\sigma_{B}}{k_{a}}\left(\frac{\partial T^{4}}{\partial y}\right)=-\frac{16}{3} \frac{\sigma_{B} T^{3}}{k_{a}}\left(\frac{\partial T}{\partial y}\right),
$$


Thermal conductivity is considered to linearly vary with temperature [30] as follows:

$$
\begin{aligned}
& k_{f}(T)=k_{\infty}\left[1+\gamma^{*}\left(\frac{T-T_{\infty}}{T_{w}-T_{\infty}}\right)\right] \\
& k_{f}(T)=k_{\infty}\left[1+\gamma^{*} \theta\right]
\end{aligned}
$$

By implementing the above variables, Eqns. (4)-(6) are transformed to the following non-similar form in a $(\xi, \eta)$ coordinate system:

$$
\begin{gathered}
{\left[1+\varepsilon\left(1-\varepsilon_{1} \xi^{2} f^{\prime \prime 2}\right)\right] f^{\prime \prime \prime}-(1+\Lambda \xi)\left(f^{\prime}\right)^{2}-\left(\frac{1}{D a}+H a\right) f^{\prime}+f f^{\prime \prime}} \\
+\frac{\sin \xi}{\xi}(\theta-N \phi)=\xi\left[\frac{\partial f^{\prime}}{\partial \xi} f^{\prime}-\frac{\partial f}{\partial \xi} f^{\prime \prime}\right] \\
\frac{1}{P_{r}}\left[\left(\left[1+\gamma^{*} \theta\right]+\frac{4}{3 R}\right) \theta^{\prime \prime}+\gamma^{*}\left(\theta^{\prime}\right)^{2}\right]+N_{b} \phi^{\prime} \theta^{\prime}+N_{t}\left(\theta^{\prime}\right)^{2}+f \theta^{\prime} \\
=\xi\left[\frac{\partial \theta}{\partial \xi} f^{\prime}-\frac{\partial f}{\partial \xi} \theta^{\prime}\right] \\
\frac{1}{S c} \phi^{\prime \prime}+\frac{N_{t}}{N_{b}} \theta^{\prime \prime}-K r \phi+f \phi^{\prime}=\xi\left[\frac{\partial \phi}{\partial \xi} f^{\prime}-\frac{\partial f}{\partial \xi} \phi^{\prime}\right]
\end{gathered}
$$

with the transformed conditions

$$
\begin{aligned}
& \eta=0: f^{\prime}=0, f=f_{w}, \theta=1, \phi=1 \\
& \eta \rightarrow \infty: f^{\prime} \rightarrow 0, \theta \rightarrow 0, \phi \rightarrow 0
\end{aligned}
$$

Where, the primes represent the differentiation with respect to $\eta, \Lambda=\Gamma a, P_{r}=\frac{\rho_{f} \nu_{f}\left(C_{p}\right)_{f}}{k_{0}}$, $D a=\frac{K \sqrt{G r}}{a^{2}}, N_{t}=\tau \frac{D_{T}\left(T_{w}-T_{\infty}\right)}{\nu_{f}}, N_{b}=\tau \frac{D_{B}\left(C_{w}-C_{\infty}\right)}{\nu_{f}}, R=\frac{k_{0} k_{a}}{4 \sigma_{B} T_{\infty}^{3}}, S c=\frac{\nu_{f}}{D_{B}}, \varepsilon=\frac{1}{\mu_{f} \beta_{1} b}$, $\varepsilon_{1}=\frac{\nu_{f}^{2}}{2 b^{2} a^{4}}, f_{w}=\frac{-V_{w} a}{\nu_{f}^{4} \sqrt[4]{G r}}, H a=\frac{\sigma_{f} B_{0}^{2} a^{2}}{\rho_{f} \nu_{f} \sqrt{G r}}, K r=\frac{K_{c} a^{2}}{\nu_{f} \sqrt{G r}}, N=\frac{\left(\rho_{p}-\rho_{f}\right)\left(C_{w}-C_{\infty}\right)}{\left(1-C_{\infty}\right) \rho_{f} \beta_{2}\left(T_{w}-T_{\infty}\right)}$ and $G r=\frac{\left(1-C_{\infty}\right) g_{a} \beta_{2}\left(T_{w}-T_{\infty}\right) a^{3}}{v_{f}^{2}}$

It is to be noted that $f_{w}>0$ with $V_{w}>0$ represents blowing and $f_{w}<0$ with $V_{w}<0$ represents suction.

The skin-friction coefficient $\left(C f_{x}\right)$, Nusselt number $\left(N u_{x}\right)$ and Sherwood number $\left(S h_{x}\right)$ at cylindrical surface is given, respectively, by:

$$
\begin{aligned}
C f_{x} G r^{-3 / 4} & =(1+\varepsilon) \xi f^{\prime \prime}(\xi, 0)-\frac{\varepsilon}{3} \varepsilon_{1}\left(\xi f^{\prime \prime}(\xi, 0)\right)^{3} \\
N u_{x} G r^{-1 / 4} & =-\left(1+\gamma^{*} \theta\right)\left(1+\frac{4}{3 R}\right) \theta^{\prime}(0) \\
S h_{x} G r^{-1 / 4} & =-\phi^{\prime}(0)
\end{aligned}
$$

\section{Computational Methodology}

The dimensionless Eqns. (11)-(13) with the appropriate boundary conditions as considered in Eqn. (14) formulate a coupled, nonlinear, partial differential boundary value problem which is numerically solved by utilizing the unconditionally stable Keller box method (KBM). This numerical scheme is second-order accurate and particularly 
versatile for parabolic type partial differential equations. The procedure to obtain solutions by using the KBM scheme is given below:

- The $n^{\text {th }}$ order dimensionless equations are transformed into $\mathrm{n}$ first order dimensionless equations..

- The transformed $\mathrm{n}$ first order equations are discretized by utilizing a central differences scheme.

- Newton's method is employed to linearize the algebraic equations.

- The results are acquired by solving the block matrix system using block tri-diagonal elimination technique.

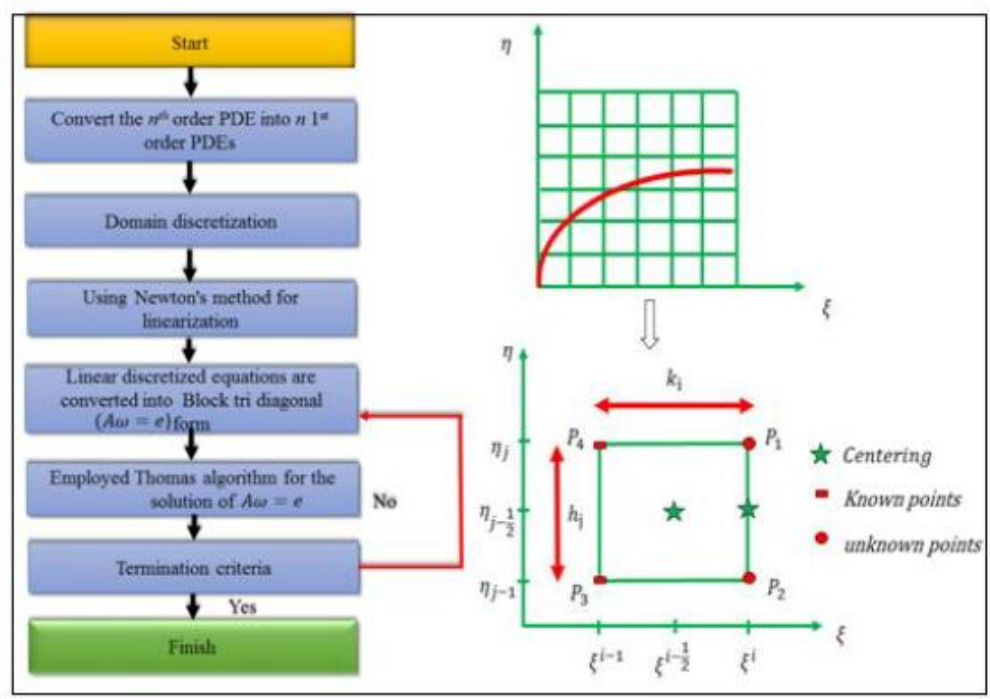

Figure 2. Numerical methodology - flow chart, boundary layer mesh and Keller box element

Step 1: First order system

We consider a new set of variables $u(\xi, \eta), v(\xi, \eta), s(\xi, \eta), t(\xi, \eta), g(\xi, \eta)$, and $p(\xi, \eta)$ to transform the $n^{\text {th }}$ order dimensionless equations into a group of first order dimensionaless equations. Let $f, u, v, s, t, g$ and $p$ be the new dependent variables as given below:

$$
f=f, f^{\prime}=u, u^{\prime}=v, \theta=s, s^{\prime}=t, \phi=g, g^{\prime}=p .
$$

By implementing the above substitutions, Eqns. (11)-(13) are transformed to:

$$
\begin{gathered}
\left(1+\varepsilon\left(1-\varepsilon_{1} \xi^{2} v^{2}\right)\right) v^{\prime}-(1+\wedge \xi) u^{2}+f v+B(s-N g) \\
-\left(\frac{1}{D a}+H a\right) u=\xi\left[u \frac{\partial u}{\partial \xi}-v \frac{\partial f}{\partial \xi}\right] \\
\frac{1}{\mathrm{P}_{r}}\left[\left(1+\gamma^{*} s\right)+\frac{4}{3 R}\right] t^{\prime}+\frac{\gamma^{*} t^{2}}{\mathrm{P}_{r}}+f t+N_{b} t p+N_{t} t^{2}=\xi\left[u \frac{\partial s}{\partial \xi}-t \frac{\partial f}{\partial \xi}\right],
\end{gathered}
$$




$$
\frac{1}{S c}\left[p^{\prime}+\frac{N_{t}}{N_{b}} t^{\prime}\right]+f p-K r g=\xi\left[u \frac{\partial g}{\partial \xi}-p \frac{\partial f}{\partial \xi}\right]
$$

with boundary conditions:

$$
\begin{aligned}
& \eta=0: f^{\prime}=0, f=f_{w}, \theta=1, \phi=1 \\
& \eta \rightarrow \infty: f^{\prime} \rightarrow 0, \theta \rightarrow 0, \phi \rightarrow 0 .
\end{aligned}
$$

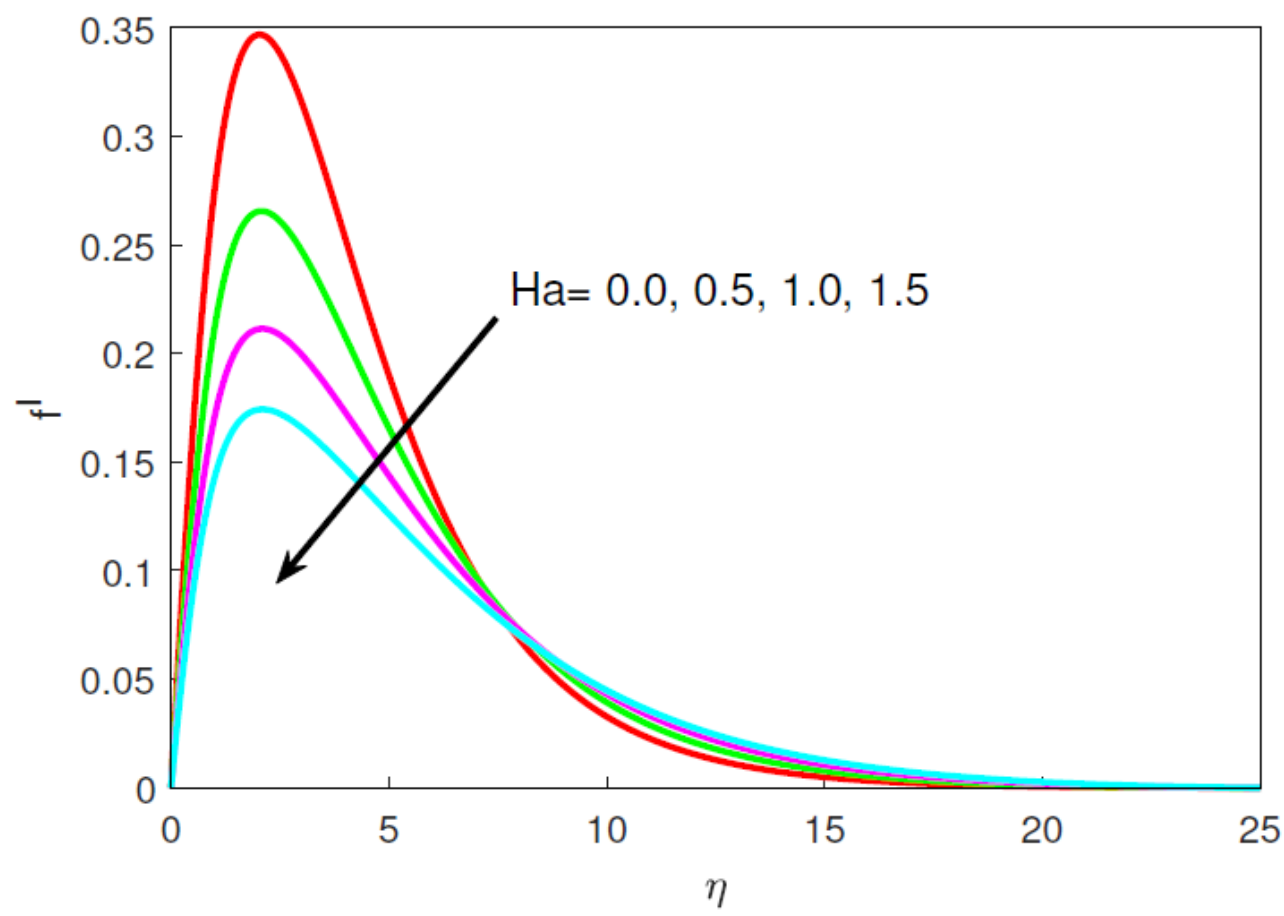

Figure 3. $f^{\prime}$ variations for enhancing $\mathrm{Ha}$

Step: 2: Difference formulation

The net point of $(\xi, \eta)$ plane is expressed in the following relations:

$$
\begin{aligned}
& \xi^{0}=0, \xi^{i}=\xi^{i-1}+k_{i}, i=1,2,3, \ldots, I, \\
& \eta_{0}=0, \eta_{j}=\eta_{j-1}+h_{j}, j=1,2,3, \ldots, J .
\end{aligned}
$$

where $k_{i}$ is $\Delta \xi$ spacing in the $i^{\text {th }}$ node and $h_{j}$ is $\Delta \eta$ spacing in $j^{\text {th }}$ node. The following discretizations are applied:

$$
\left(\frac{\partial()}{\partial \xi}\right)_{j-\frac{1}{2}}^{i-\frac{1}{2}}=\frac{()_{j-\frac{1}{2}}^{i}-()_{j-\frac{1}{2}}^{i-1}}{k_{i}}, \quad\left(\frac{\partial()}{\partial \eta}\right)_{j-\frac{1}{2}}^{i-\frac{1}{2}}=\frac{()_{j-\frac{1}{2}}^{i}-()_{j-\frac{1}{2}}^{i-1}}{h_{j}}
$$

At the midpoint $\left(\xi^{i}, \eta_{j-\frac{1}{2}}\right)$ between the segments $\left(\xi^{i}, \eta_{j-1}\right)\left(\xi^{i}, \eta_{j}\right)$ the following 


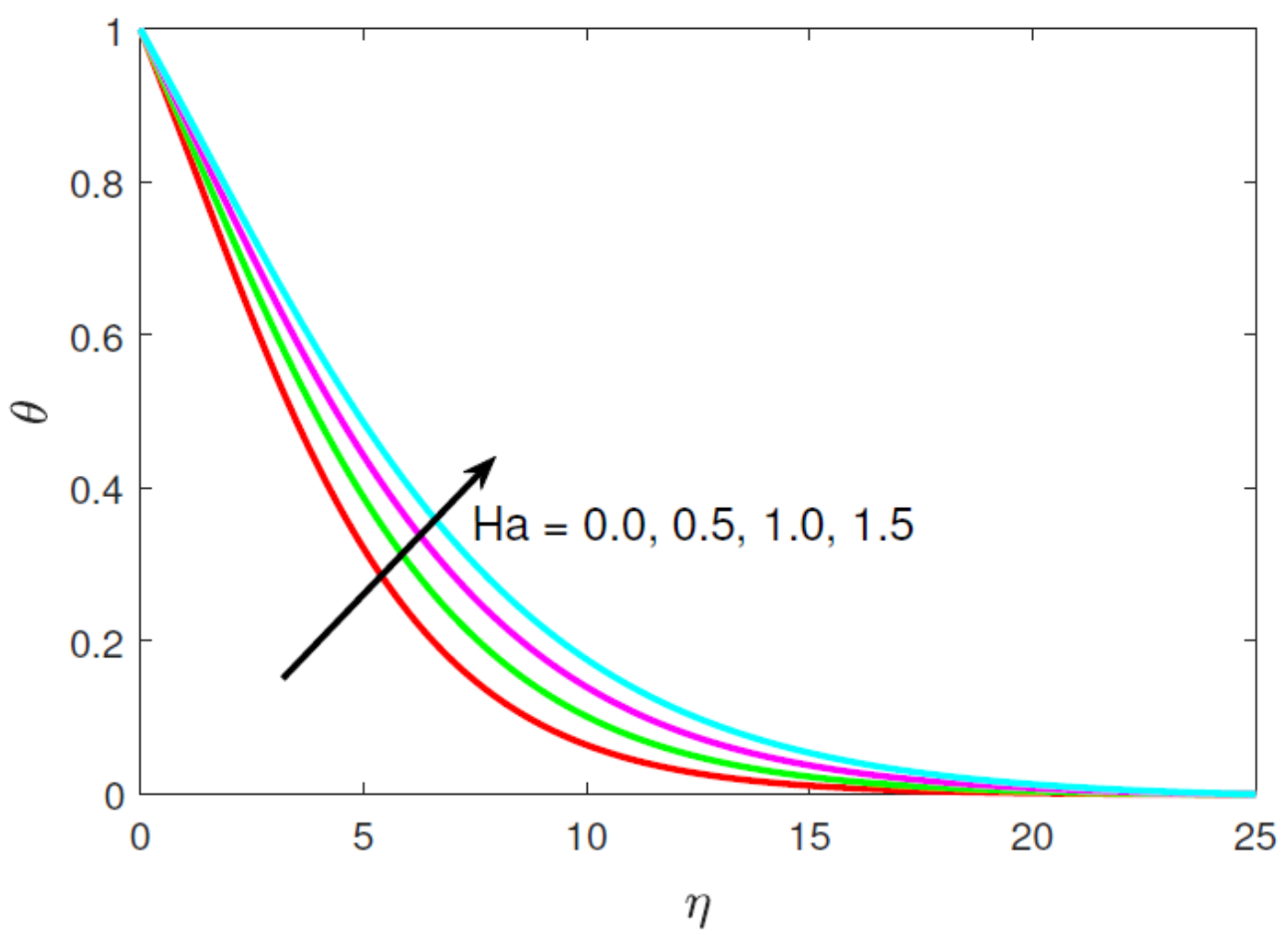

Figure 4. $\theta$ variations for enhancing $\mathrm{Ha}$.

central difference approximations are deployed:

$$
\begin{aligned}
& f^{\prime}=u \Rightarrow u_{j-\frac{1}{2}}^{i}=\frac{u_{j}^{i}+u_{j-1}^{i}}{2}=\frac{\left(f_{j}^{i}-f_{j-1}^{i}\right)}{h_{j}}, \\
& u^{\prime}=v \Rightarrow v_{j-\frac{1}{2}}^{i}=\frac{v_{j}^{i}+v_{j-1}^{i}}{2}=\frac{\left(u_{j}^{i}-u_{j-1}^{i}\right)}{h_{j}}, \\
& s^{\prime}=t \Rightarrow t_{j-\frac{1}{2}}^{i}=\frac{t_{j}^{i}+t_{j-1}^{i}}{2}=\frac{\left(s_{j}^{i}-s_{j-1}^{i}\right)}{h_{j}}, \\
& g^{\prime}=p \Rightarrow p_{j-\frac{1}{2}}^{i}=\frac{p_{j}^{i}+p_{j-1}^{i}}{2}=\frac{\left(g_{j}^{i}-g_{j-1}^{i}\right)}{h_{j}},
\end{aligned}
$$

The first order partial differential equations (19) to (21) are approximated by centering at $\left(\xi^{i-\frac{1}{2}}, \eta_{j--\frac{1}{2}}\right)$ of the rectangle points (P1, P2, P3, and P4) which give the following equations:

$$
\begin{aligned}
& \left(v^{\prime}\right)^{i}+\varepsilon\left(v^{\prime}\right)^{i}-\varepsilon \varepsilon_{1} \xi^{2}\left(v^{2} v^{\prime}\right)^{i}+(1+\alpha)(f v)^{i}-(1+\alpha+\xi \Lambda)\left(u^{2}\right)^{i} \\
& -\left(H a+\frac{1}{D a}\right) u^{i}+\alpha v^{i-1} f^{i}-\alpha f^{i-1} v^{i}+B\left(s^{i}-N g^{i}\right)=\left[E_{1}\right]_{j-\frac{1}{2}}^{i-1}
\end{aligned}
$$




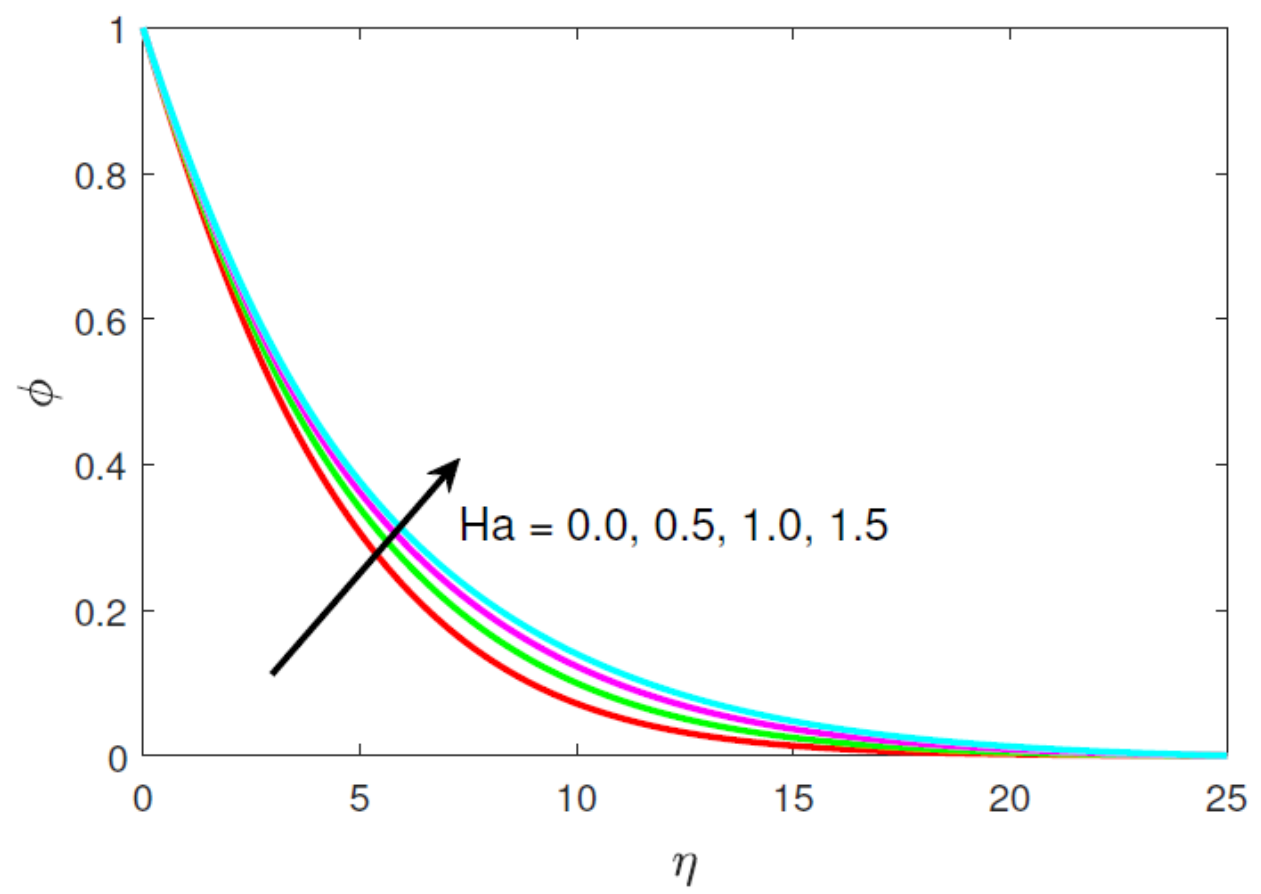

Figure 5. $\phi$ variations for enhancing $\mathrm{Ha}$.

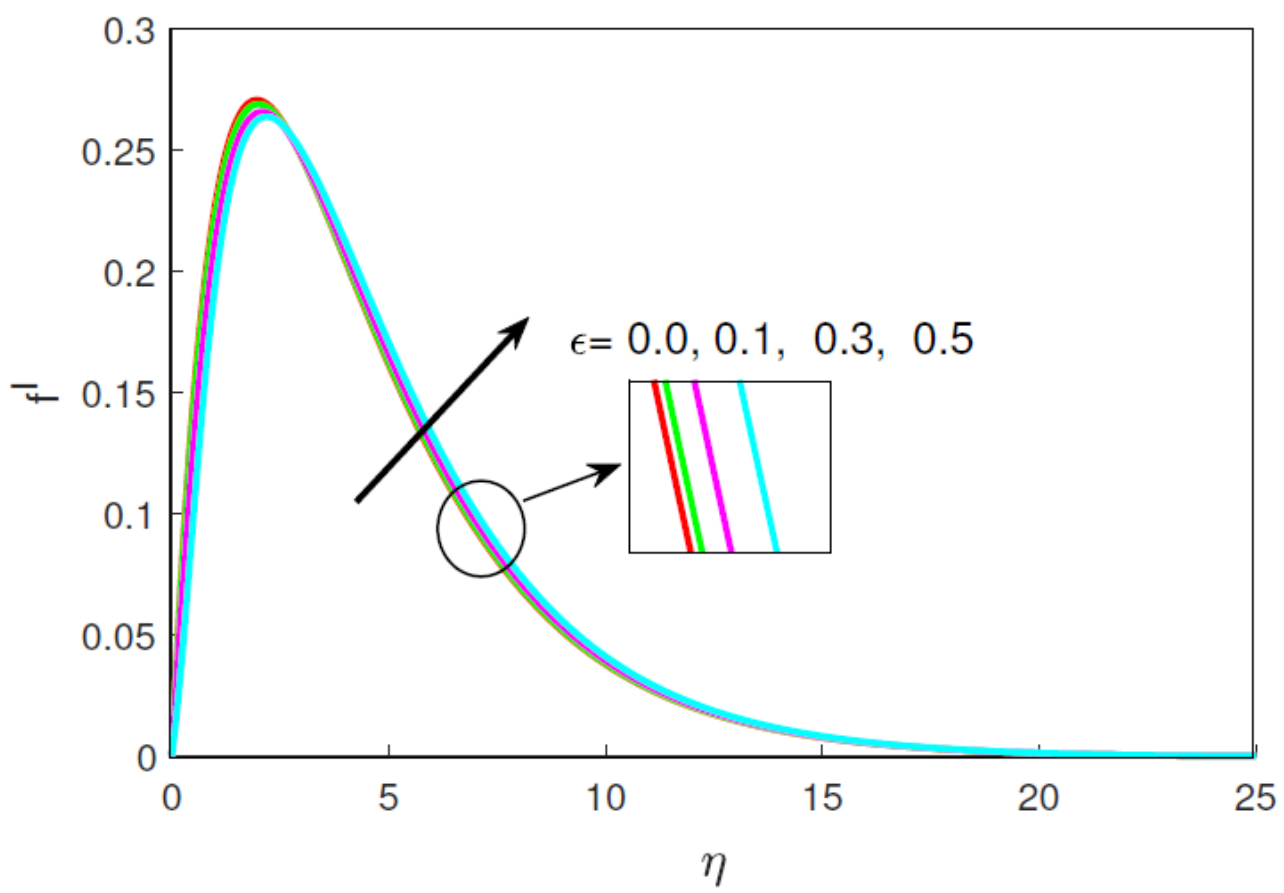

Figure 6. $f^{\prime}$ variations for enhancing $\varepsilon$ 


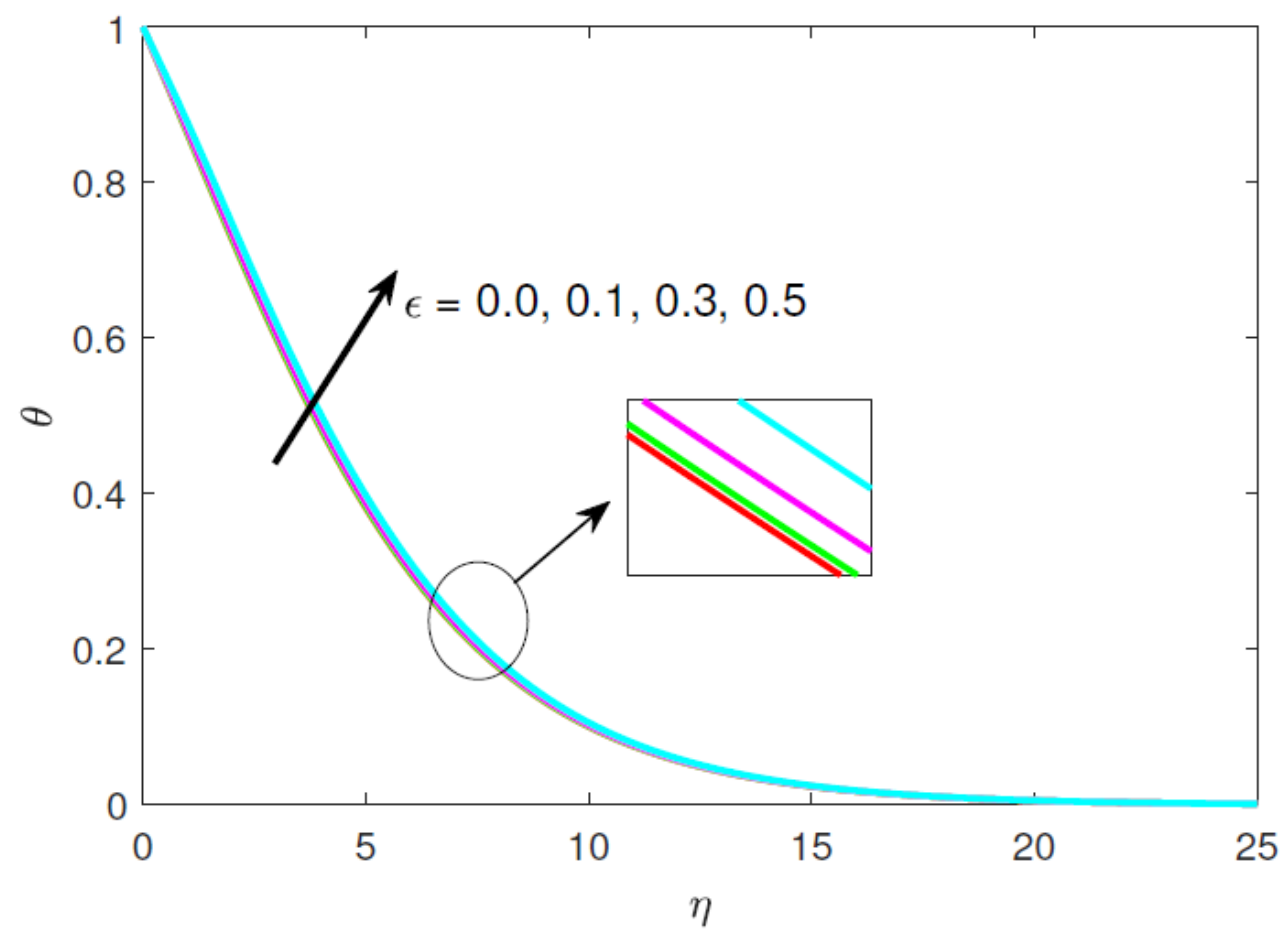

Figure 7. $\theta$ variations for enhancing $\varepsilon$

$$
\begin{gathered}
\frac{1}{\mathrm{P}_{r}}\left[1+\gamma^{*} s^{i}\right]\left(t^{\prime}\right)^{i}+\frac{4}{P_{r} 3 R}\left(t^{\prime}\right)^{i}+\frac{\gamma^{*}}{P_{r}}\left(t^{2}\right)^{i}+N_{b}(t p)^{i}+N_{t}\left(t^{2}\right)-\alpha(u s)^{i} \\
+(1+\alpha)(f t)^{i}+\alpha s^{i-1} u^{i}-\alpha u^{i-1} s^{i}-\alpha f^{i-1} t^{i}+\alpha t^{i-1} f^{i}=\left[E_{2}\right]_{j-\frac{1}{2}}^{i-1} \\
\frac{1}{S c}\left(p^{\prime}\right)^{i}+\frac{N_{t}}{N_{b}}\left(t^{\prime}\right)^{i}+(1+\alpha)(f p)^{n}-\alpha(u g)^{i}+\alpha g^{i-1} u^{i}-\alpha u^{i-1} g^{i} \\
-\alpha f^{i-1} p^{i}+\alpha p^{i-1} f^{i}-K r g^{i}=\left[E_{3}\right]_{j-\frac{1}{2}}
\end{gathered}
$$

where, $\alpha=\frac{\xi^{i-1 / 2}}{k_{n}}$ and $B=\frac{\sin \left(\xi^{i-1 / 2}\right)}{\xi^{i-1 / 2}}$

At $\xi=\xi^{i}$, the boundary conditions becomes

$$
f_{0}^{i}=u_{0}^{i}=0, s_{0}^{i}=1, g_{0}^{i}=1, u_{J}^{i}=0, s_{J}^{i}=0, g_{J}^{i}=0
$$

Step: 3: Newton's method

The unknowns $\left(f_{j}^{i}, u_{j}^{i}, v_{j}^{i}, g_{j}^{i}, p_{j}^{i}, s_{j}^{i}, t_{j}^{i}\right)$ are calculated with the help of knowns $f_{j}^{i-1}, u_{j}^{i-1}, v_{j}^{i-1}, g_{j}^{i-1}, p_{j}^{i-1}, s_{j}^{i-1}, t_{j}^{i-1}$ where, $0 \leq j \leq J$.

$$
\left(f_{j}^{i}, u_{j}^{i}, v_{j}^{i}, g_{j}^{i}, p_{j}^{i}, s_{j}^{i}, t_{j}^{i}\right) \equiv\left(f_{j}, u_{j}, v_{j}, g_{j}, p_{j}, s_{j}, t_{j}\right) .
$$

The set of central difference equations can be expressed as

$$
\frac{u_{j}+u_{j-1}}{2}=\frac{f_{j}-f_{j-1}}{h_{j}},
$$




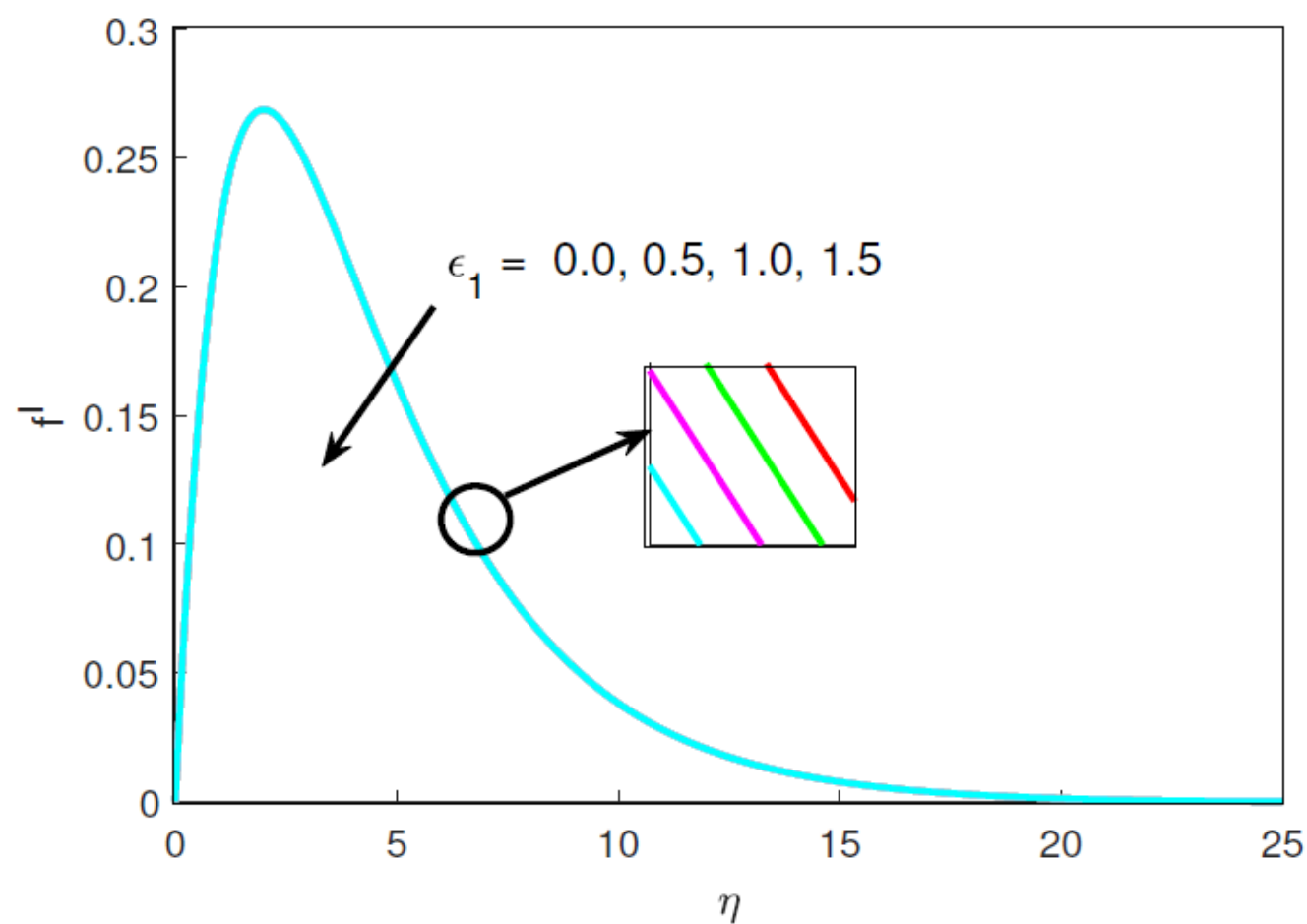

Figure 8. $f^{\prime}$ variations for enhancing $\varepsilon_{1}$.

$$
\begin{aligned}
& \frac{v_{j}+v_{j-1}}{2}=\frac{\left(u_{j}-u_{j-1}\right)}{h_{j}}, \\
& \frac{t_{j}+t_{j-1}}{2}=\frac{\left(s_{j}-s_{j-1}\right)}{h_{j}}, \\
& \frac{p_{j}+p_{j-1}}{2}=\frac{\left(g_{j}-g_{j-1}\right)}{h_{j}}, \\
& \left.\begin{array}{c}
{\left[1+\varepsilon-\frac{\varepsilon \varepsilon_{1} \xi^{2}}{4}\left(v_{j}+v_{j-1}\right)^{2}\right]\left(v_{j}-v_{j-1}\right)+\frac{\alpha h_{j}}{2} v_{j-1 / 2}^{i-1}\left(f_{j}+f_{j-1}\right)} \\
+\frac{(1+\alpha) h_{j}}{4}\left[\left(f_{j}+f_{j-1}\right)\left(v_{j}+v_{j-1}\right)\right]-\frac{h_{j}}{2}\left(H a+\frac{1}{D a}\right)\left(u_{j}+u_{j-1}\right) \\
\frac{h_{j}}{4}(1+\alpha+\xi \Lambda)\left(u_{j}+u_{j-1}\right)^{2}-\frac{\alpha h_{j}}{2} f_{j-1 / 2}^{i-1}\left(v_{j}+v_{j-1}\right) \\
+\frac{B h_{j}}{2}\left[s_{j}+s_{j-1}-N\left(g_{j}+g_{j-1}\right)\right]=\left[E_{1}\right]_{j-1 / 2}^{i-1}
\end{array}\right\} \\
& \frac{1}{\mathrm{P}_{r}}\left[\left(1+\gamma^{*}\left(s_{j}+s_{j-1}\right)\right)\left(t_{j}-t_{j-1}\right)\right]+\frac{4}{3 P_{r} R}\left(t_{j}-t_{j-1}\right) \\
& +\frac{\gamma^{*}}{4 P_{r}}\left(t_{j}+t_{j-1}\right)^{2}+\frac{h_{j}}{4} N_{b}\left(\left(t_{j}+t_{j-1}\right)\left(p_{j}+p_{j-1}\right)\right) \\
& +\frac{(1+\alpha) h_{j}}{4}\left[\left(f_{j}+f_{j-1}\right)\left(t_{j}+t_{j-1}\right)\right]-\frac{\alpha h_{j}}{4}\left[\left(u_{j}+u_{j-1}\right)\left(s_{j}+s_{j-1}\right)\right] \\
& +\frac{\alpha h_{j}}{2} s_{j-1 / 2}^{i-1}\left(u_{j}+u_{j-1}\right)-\frac{\alpha h_{j}}{2} u_{j-1 / 2}^{i-1}\left(s_{j}+s_{j-1}\right) \\
& -\frac{\alpha h_{j}}{2} f_{j-1 / 2}^{i-1}\left(t_{j}+t_{j-1}\right)+\frac{h_{j}}{4} N_{t}\left(t_{j}+t_{j-1}\right)^{2} \\
& +\frac{\alpha h_{j}}{2} t_{j-1 / 2}^{i-1}\left(f_{j}+f_{j-1}\right)=\left[E_{2}\right]_{j-1 / 2}^{i-1}
\end{aligned}
$$




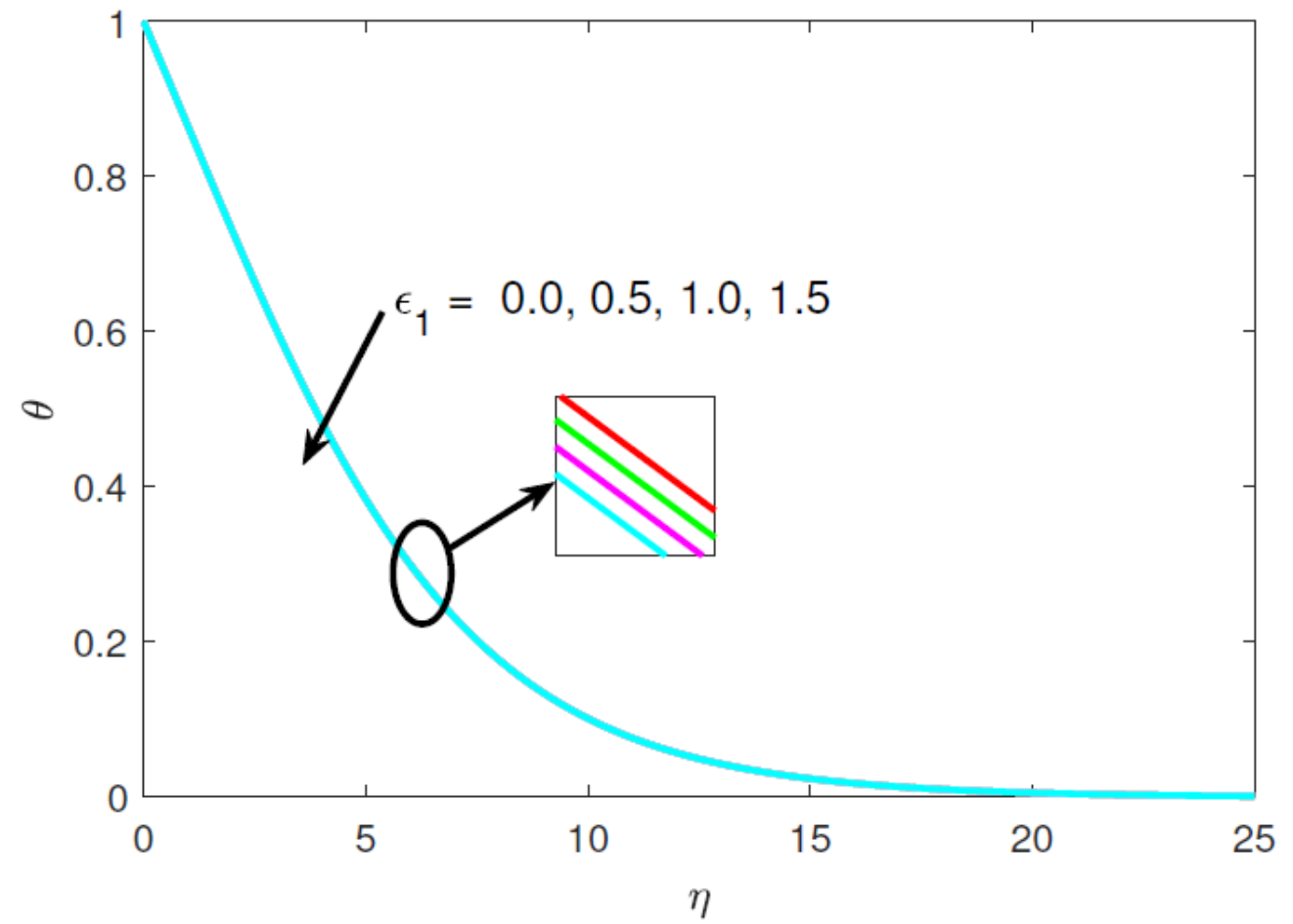

Figure 9. $\theta$ variations for enhancing $\varepsilon_{1}$.

$$
\begin{aligned}
& \frac{1}{S_{c}}\left(p_{j}-p_{j-1}\right)+\frac{N_{t}}{S_{c N}}\left(t_{j}+t_{j-1}\right)-\frac{K r h_{j}}{2}\left(g_{j}+g_{j-1}\right) \\
& +\frac{(1+\alpha) h_{j}}{4}\left[\left(f_{j}+f_{j-1}\right)\left(p_{j}+p_{j-1}\right)\right]-\frac{\alpha h_{j}}{2} u_{j-1 / 2}^{i-1}\left(g_{j}+g_{j-1}\right) \\
& +\frac{\alpha h_{j}}{2} g_{j-1 / 2}^{i-1}\left(u_{j}+u_{j-1}\right)-\frac{\alpha h_{j}}{4}\left[\left(u_{j}+u_{j-1}\right)\left(g_{j}+g_{j-1}\right)\right] \\
& \left.-\frac{\alpha h_{j}}{2} f_{j-1 / 2}^{i-1}\left(p_{j}+p_{j-1}\right)+\frac{\alpha h_{j}}{2} p_{j-1 / 2}^{i-1}\left(f_{j}+f_{j-1}\right)=\left[E_{3}\right]_{j-1 / 2}^{i-1}\right) \\
& {\left[E_{1}\right]_{j-\frac{1}{2}}^{i-1}=h_{j}\left[\begin{array}{l}
-\left[(1+\varepsilon)\left(\frac{v_{j}-v_{j-1}}{h_{j}}\right)-\varepsilon \varepsilon_{1} \xi^{2}\left(\frac{v_{j}-v_{j-1}}{h_{j}}\right)\left(v_{j-\frac{1}{2}}\right)^{2}\right] \\
+(\alpha-1)\left(f_{j-\frac{1}{2}} v_{j-\frac{1}{2}}\right)+(1-\alpha+\xi \Lambda)\left(u_{j-\frac{1}{2}}\right)^{2} \\
-B\left(s_{j-\frac{1}{2}}-N g_{j-\frac{1}{2}}\right)+\left(H a+\frac{1}{D a}\right) u_{j-\frac{1}{2}}
\end{array}\right]} \\
& {\left[E_{2}\right]_{j-\frac{1}{2}}^{i-1}=h_{j}\left[\begin{array}{r}
-\left(\begin{array}{r}
\frac{1}{\mathrm{P}_{r}}\left[1+\gamma^{*} s_{j-\frac{1}{2}}\right]\left(\frac{t_{j}-t_{j-1}}{h_{j}}\right)+\frac{4}{P_{r} 3 R}\left(\frac{t_{j}-t_{j-1}}{h_{j}}\right) \\
+\frac{\gamma^{*}}{P_{r}}\left(t_{j-\frac{1}{2}}\right)^{2}+N_{b}\left(t_{j-\frac{1}{2}} p_{j-\frac{1}{2}}\right)+N_{t}\left(t_{j-\frac{1}{2}}\right)^{2}
\end{array}\right) \\
+(\alpha-1)\left(f_{j-\frac{1}{2}} t_{j-\frac{1}{2}}\right)-\alpha\left(u_{j-\frac{1}{2}} s_{j-\frac{1}{2}}\right)
\end{array}\right]} \\
& {\left[E_{3}\right]_{j-\frac{1}{2}}^{i-1}=h_{j}\left[\begin{array}{l}
-\frac{1}{S c}\left(\frac{p_{j}-p_{j-1}}{h_{j}}\right)-\frac{N_{t}}{S c N_{b}}\left(\frac{t_{j}-t_{j-1}}{h_{j}}\right) \\
+(\alpha-1)\left(f_{j-\frac{1}{2}} p_{j-\frac{1}{2}}\right)-\alpha\left(u_{j-\frac{1}{2}} g_{j-\frac{1}{2}}\right)+K r g_{j-\frac{1}{2}}
\end{array}\right]}
\end{aligned}
$$

Here $\left[E_{1}\right]_{j-1 / 2}^{i-1},\left[E_{2}\right]_{j-1 / 2}^{i-1}$, and $\left[E_{3}\right]_{j-1 / 2}^{i-1}$ are the known quantities. Newton's method is employed to linearize the system of nonlinear equations with the following iterates:

$$
\begin{aligned}
& \omega f_{j}^{(n)}=f_{j}^{(n+1)}-f_{j}^{(n)}, \omega u_{j}^{(n)}=u_{j}^{(n+1)}-u_{j}^{(n)}, \omega v_{j}^{(n)}=v_{j}^{(n+1)}-v_{j}^{(n)}, \\
& \omega g_{j}^{(n)}=g_{j}^{(n+1)}-g_{j}^{(n)}, \omega p_{j}^{(n)}=p_{j}^{(n+1)}-p_{j}^{(n)},
\end{aligned}
$$




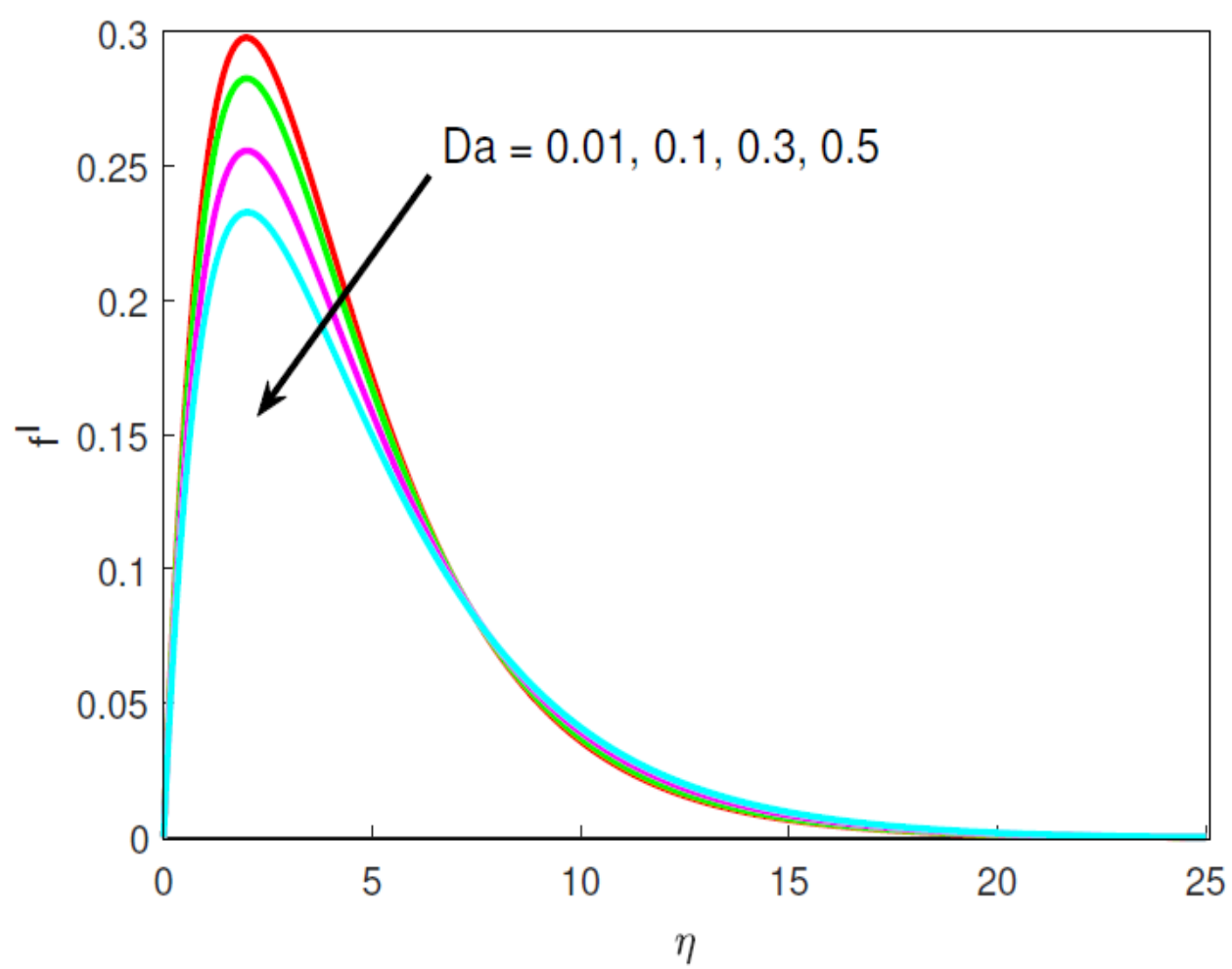

Figure 10. $f^{\prime}$ variations for enhancing $D a$.

$$
\omega s_{j}^{(n)}=s_{j}^{(n+1)}-s_{j}^{(n)}, \omega t_{j}^{(n)}=t_{j}^{(n+1)}-t_{j}^{(n)} .
$$

Implementing the above expressions in Eqns. (34)-(40) and neglecting higher-order terms of $\omega$, leads to:

$$
\begin{aligned}
& \omega f_{j}-\omega f_{j-1}-\frac{h_{j}}{2}\left(\omega u_{j}+\omega u_{j-1}\right)=\left(e_{1}\right)_{j-1 / 2}, \\
& \omega u_{j}-\omega u_{j-1}-\frac{h_{j}}{2}\left(\omega v_{j}+\omega v_{j-1}\right)=\left(e_{2}\right)_{j-1 / 2}, \\
& \omega g_{j}-\omega g_{j-1}-\frac{h_{j}}{2}\left(\omega p_{j}+\omega p_{j-1}\right)=\left(e_{3}\right)_{j-1 / 2}, \\
& \omega s_{j}-\omega s_{j-1}-\frac{h_{j}}{2}\left(\omega t_{j}+\omega t_{j-1}\right)=\left(e_{4}\right)_{j-1 / 2}, \\
& \left(a_{1}\right)_{j} \omega v_{j}+\left(a_{2}\right)_{j} \omega v_{j-1}+\left(a_{3}\right)_{j} \omega f_{j}+\left(a_{4}\right)_{j} \omega f_{j-1} \\
& \quad+\left(a_{5}\right)_{j} \omega u_{j}+\left(a_{6}\right)_{j} \omega u_{j-1}+\left(a_{7}\right)_{j} \omega s_{j}+\left(a_{8}\right)_{j} \omega s_{j-1} \\
& \quad+\left(a_{9}\right)_{j} \omega g_{j}+\left(a_{10}\right)_{j} \omega g_{j-1}=\left(e_{5}\right)_{j-1 / 2}, \\
& \left(b_{1}\right)_{j} \omega t_{j}+\left(b_{2}\right)_{j} \omega t_{j-1}+\left(b_{3}\right)_{j} \omega f_{j}+\left(b_{4}\right)_{j} \omega f_{j-1} \\
& \quad+\left(b_{5}\right)_{j} \omega u_{j}+\left(b_{6}\right)_{j} \omega u_{j-1}+\left(b_{7}\right)_{j} \omega s_{j}+\left(b_{8}\right)_{j} \omega s_{j-1} \\
& \quad+\left(b_{9}\right)_{j} \omega p_{j}+\left(b_{10}\right)_{j} \omega p_{j-1}=\left(e_{6}\right)_{j-1 / 2},
\end{aligned}
$$




$$
\begin{aligned}
& \left(c_{1}\right)_{j} \omega p_{j}+\left(c_{2}\right)_{j} \omega p_{j-1}+\left(c_{3}\right)_{j} \omega f_{j}+\left(c_{4}\right)_{j} \omega f_{j-1} \\
& \quad+\left(c_{5}\right)_{j} \omega u_{j}+\left(c_{6}\right)_{j} \omega u_{j-1}+\left(c_{7}\right)_{j} \omega g_{j}+\left(c_{8}\right)_{j} \omega g_{j-1} \\
& \quad+\left(c_{9}\right)_{j} \omega t_{j}+\left(c_{10}\right)_{j} \omega t_{j-1}=\left(e s_{7}\right)_{j-1 / 2},
\end{aligned}
$$

Here:

$$
\begin{aligned}
& \left(a_{1}\right)_{j}=1+\varepsilon-\varepsilon \varepsilon_{1} \xi^{2}\left(v_{j-1 / 2}\right)^{2}+h_{j}\left[\frac{(1+\alpha)}{2} f_{j-1 / 2}-\frac{\alpha}{2} f_{j-1 / 2}^{n-1}\right], \\
& \left(a_{2}\right)_{j}=-1-\varepsilon+\varepsilon \varepsilon_{1} \xi^{2}\left(v_{j-1 / 2}\right)^{2}+h_{j}\left[\frac{(1+\alpha)}{2} f_{j-1 / 2}-\frac{\alpha}{2} f_{j-1 / 2}^{n-1}\right], \\
& \left(a_{3}\right)_{j}=h_{j}\left[\frac{(1+\alpha)}{2} v_{j-1 / 2}+\frac{\alpha}{2} v_{j-1 / 2}^{n-1}\right],\left(a_{4}\right)_{j}=\left(a_{3}\right)_{j}, \\
& \left(a_{5}\right)_{j}=h_{j}\left[-(1+\alpha+\xi \Lambda) u_{j-1 / 2}-\frac{1}{2}\left(H a+\frac{1}{D a}\right)\right],\left(a_{6}\right)_{j}=\left(a_{5}\right)_{j} \\
& \left(a_{7}\right)_{j}=\frac{B}{2} h_{j},\left(a_{8}\right)_{j}=\left(a_{7}\right)_{j}, \\
& \left(a_{9}\right)_{j}=-\frac{B}{2} h_{j} N,\left(a_{10}\right)_{j}=\left(a_{9}\right)_{j}, \\
& \left(b_{1}\right)_{j}=\frac{1}{\mathrm{P}_{r}}\left[1+\frac{4}{3 R}+\gamma^{*} s_{j-1 / 2}\right]+h_{j}\left[\begin{array}{l}
\frac{\gamma^{*}}{\mathrm{P}_{r}} t_{j-1 / 2}+\frac{N_{b}}{2} p_{j-1 / 2}+N_{t} t_{j-1 / 2} \\
+\frac{(1+\alpha)}{2} f_{j-1 / 2}-\frac{\alpha}{2} f_{j-1 / 2}^{n-1}
\end{array}\right], \\
& \left(b_{2}\right)_{j}=-\frac{1}{\mathrm{P}_{r}}\left[1+\frac{4}{3 R}+\gamma^{*} s_{j-1 / 2}\right]+h_{j}\left[\begin{array}{l}
\frac{\gamma^{*}}{\mathrm{P}_{r}} t_{j-1 / 2}+\frac{N_{b}}{2} p_{j-1 / 2}+N_{t} t_{j-1 / 2} \\
+\frac{(1+\alpha)}{2} f_{j-1 / 2}-\frac{\alpha}{2} f_{j-1 / 2}^{n-1}
\end{array}\right], \\
& \left(b_{3}\right)_{j}=h_{j}\left[\frac{(1+\alpha)}{2} t_{j-1 / 2}+\frac{\alpha}{2} t_{j-1 / 2}^{n-1}\right],\left(b_{4}\right)_{j}=\left(b_{3}\right)_{j} \text {, } \\
& \left(b_{5}\right)_{j}=h_{j}\left[-\frac{\alpha}{2} s_{j-1 / 2}+\frac{\alpha}{2} s_{j-1 / 2}^{n-1}\right],\left(b_{6}\right)_{j}=\left(b_{5}\right)_{j}, \\
& \left(b_{7}\right)_{j}=\frac{\gamma^{*}}{2 \mathrm{P}_{r}}\left(t_{j}-t_{j-1}\right)+h_{j}\left[-\frac{\alpha}{2} u_{j-1 / 2}-\frac{\alpha}{2} u_{j-1 / 2}^{n-1}\right],\left(b_{8}\right)_{j}=\left(b_{7}\right)_{j}, \\
& \left(b_{9}\right)_{j}=h_{j}\left[\frac{N_{b}}{2} t_{j-1 / 2}\right],\left(b_{10}\right)_{j}=\left(b_{9}\right)_{j} \\
& \left(c_{1}\right)_{j}=\frac{1}{S c}+h_{j}\left[\frac{(1+\alpha)}{2} f_{j-1 / 2}-\frac{\alpha}{2} f_{j-1 / 2}^{n-1}\right] \text {, } \\
& \left(c_{2}\right)_{j}=-\frac{1}{S c}+h_{j}\left[\frac{(1+\alpha)}{2} f_{j-1 / 2}-\frac{\alpha}{2} f_{j-1 / 2}^{n-1}\right] \text {, } \\
& \left(c_{3}\right)_{j}=h_{j}\left[\frac{(1+\alpha)}{2} p_{j-1 / 2}+\frac{\alpha}{2} p_{j-1 / 2}^{n-1}\right],\left(c_{4}\right)_{j}=\left(c_{3}\right)_{j}, \\
& \left(c_{5}\right)_{j}=h_{j}\left[-\frac{\alpha}{2} g_{j-1 / 2}+\frac{\alpha}{2} g_{j-1 / 2}^{n-1}\right],\left(c_{6}\right)_{j}=\left(c_{5}\right)_{j}, \\
& \left(c_{7}\right)_{j}=h_{j}\left[\frac{K r}{2}-\frac{\alpha}{2} u_{j-1 / 2}-\frac{\alpha}{2} u_{j-1 / 2}^{n-1}\right],\left(c_{8}\right)_{j}=\left(c_{7}\right)_{j}, \\
& \left(c_{9}\right)_{j}=\frac{1}{S c} \frac{N_{t}}{N_{b}},\left(c_{10}\right)_{j}=-\left(c_{9}\right)_{j}, \\
& \left(e_{1}\right)_{j-1 / 2}=f_{j-1}-f_{j}+h_{j} u_{j-1 / 2} \text {, } \\
& \left(e_{2}\right)_{j-1 / 2}=u_{j-1}-u_{j}+h_{j} v_{j-1 / 2} \text {, }
\end{aligned}
$$




$$
\begin{aligned}
& \left(e_{3}\right)_{j-1 / 2}=g_{j-1}-g_{j}+h_{j} p_{j-1 / 2}, \\
& \left(e_{4}\right)_{j-1 / 2}=s_{j-1}-s_{j}+h_{j} t_{j-1 / 2}, \\
& \left(e_{5}\right)_{j-1 / 2}=\left(v_{j-1}-v_{j}\right)\left[1+\varepsilon-\varepsilon \varepsilon_{1} \xi^{2}\left(v_{j-1 / 2}\right)^{2}\right]+h_{j}(1+\alpha+\wedge \xi) u_{j-1 / 2}^{2} \\
& \quad-(1+\alpha) h_{j} f_{j-1 / 2} v_{j-1 / 2}+\left(\frac{1}{D a}+H a\right) h_{j} u_{j-1 / 2}-\alpha h_{j} v_{j-1 / 2}^{n-1} f_{j-1 / 2} \\
& \quad+\alpha h_{j} f_{j-1 / 2}^{n-1} v_{j-1 / 2}-B h_{j}\left[s_{j-1 / 2}-N\left(g_{j-1 / 2}\right)\right]+\left(E_{1}\right)_{j-1 / 2}^{i-1}, \\
& \left(e_{6}\right)_{j-1 / 2}=\frac{1}{\mathrm{P}_{r}}\left(t_{j-1}-t_{j}\right)\left[1+\frac{4}{3 R_{a}}+\gamma^{*} s_{j-1 / 2}\right]-\frac{\gamma^{*}}{\mathrm{P}_{r}} h_{j}\left(t_{j-1 / 2}\right)^{2} \\
& \quad-N_{b} h_{j} t_{j-1 / 2} p_{j-1 / 2}-N_{t} h_{j}\left(t_{j-1 / 2}\right)^{2}-(1+\alpha) h_{j} f_{j-1 / 2} t_{j-1 / 2} \\
& \quad+h_{j} \alpha u_{j-1 / 2} s_{j-1 / 2}-\alpha h_{j} s_{j-1 / 2}^{n-1} u_{j-1 / 2}+\alpha h_{j} u_{j-1 / 2}^{n-1} s_{j-1 / 2} \\
& \quad+\alpha h_{j} f_{j-1 / 2}^{n-1} t_{j-1 / 2}-\alpha h_{j} t_{j-1 / 2}^{n-1} f_{j-1 / 2}+\left(E_{2}\right)_{j-1 / 2}^{i-1}, \\
& \left(e_{7}\right)_{j-1 / 2}=\frac{1}{S_{c}}\left(p_{j-1}-p_{j}\right)+\frac{1}{S c} \frac{N_{t}}{N_{b}}\left(t_{j-1}-t_{j}\right)+K r h_{j} g_{j-1 / 2} \\
& \quad-(1+\alpha) h_{j} f_{j-1 / 2} p_{j-1 / 2}+h_{j} \alpha u_{j-1 / 2} g_{j-1 / 2}-\alpha h_{j} g_{j-1 / 2}^{n-1} u_{j-1 / 2} \\
& \quad+\alpha h_{j} u_{j-1 / 2}^{n-1} g_{j-1 / 2}+\alpha h_{j} f_{j-1 / 2}^{n-1} p_{j-1 / 2} \\
& \quad-\alpha h_{j} p_{j-1 / 2}^{n-1} f_{j-1 / 2}+\left(E_{3}\right)_{j-1 / 2}^{i-1} .
\end{aligned}
$$

The boundary conditions (32) emerge as:

$$
\begin{aligned}
& \omega f_{0}=0, \omega u_{0}=0, \omega s_{0}^{n}=0, \omega g_{0}=0, \omega u_{J}=0, \omega s_{J}=0, \omega g_{J}=0 . \\
& f_{0}^{i}=u_{0}^{i}=0, s_{0}^{i}=1, g_{0}^{i}=1, u_{J}^{i}=0, s_{J}^{i}=0, g_{J}^{i}=0 .
\end{aligned}
$$

Step 4: The block tridiagonal structure

The linearized difference Eqns. (45)-(51) are computed by employing the blockelimination technique as outlined by Cebeci and Bradshaw [31] using the matrix-vector form,

$$
[A][\omega]=[e] .
$$

$$
A=\left[\begin{array}{cccccc}
{\left[A_{1}\right]} & {\left[C_{1}\right]} & & & & \\
{\left[B_{2}\right]} & {\left[A_{2}\right]} & {\left[C_{2}\right]} & & & \\
& & & \ddots & & \\
& & \ddots & & & \\
& & \ddots & & & \\
& & & {\left[B_{J-1}\right]} & {\left[A_{J-1}\right]} & {\left[C_{J-1}\right]} \\
& & & & {\left[B_{J}\right]} & {\left[C_{J}\right]}
\end{array}\right],
$$




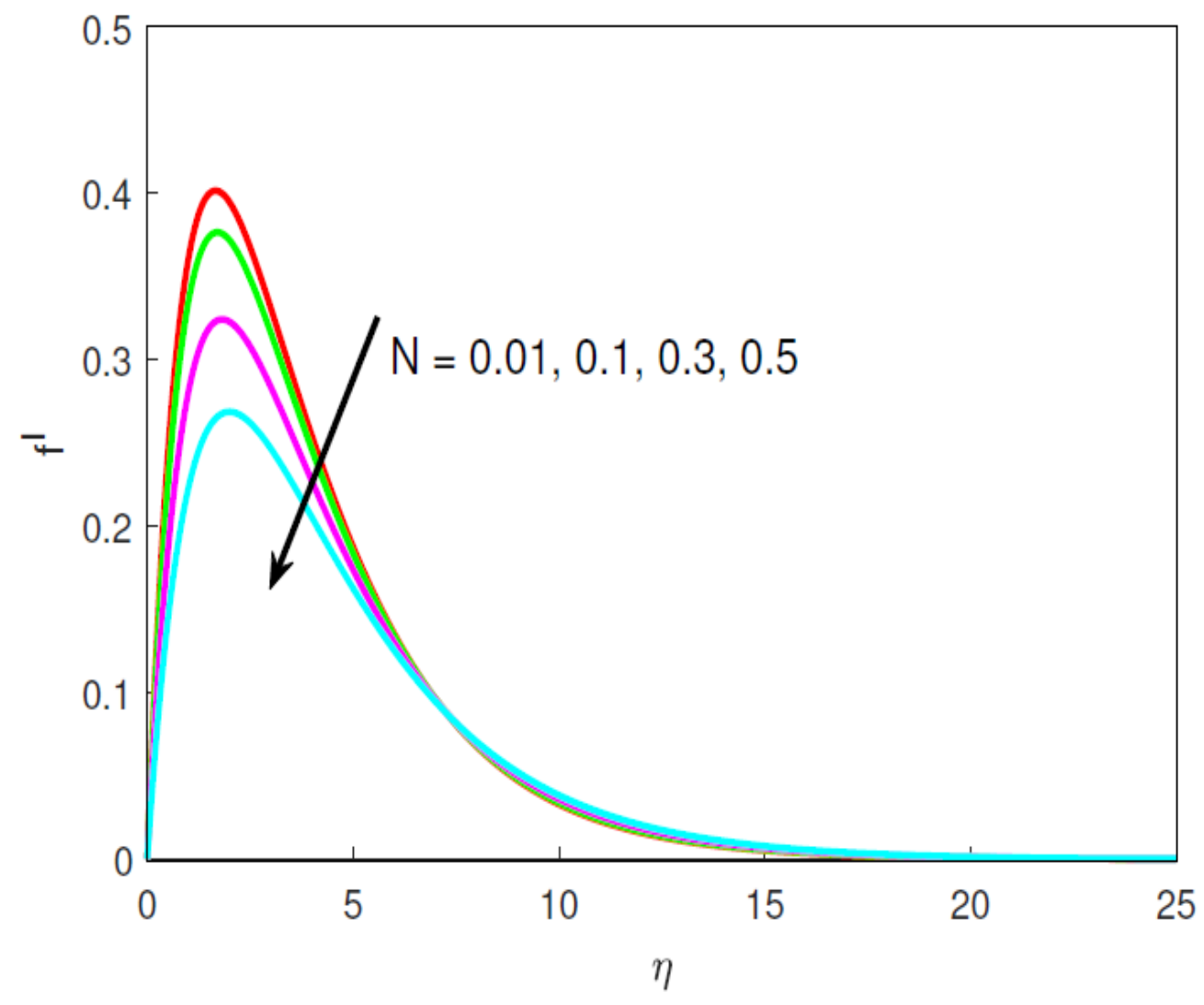

Figure 11. $f^{\prime}$ Variations for enhancing $N$.

$$
\omega=\left[\begin{array}{c}
{\left[\omega_{1}\right]} \\
{\left[\omega_{2}\right]} \\
\vdots \\
\\
{\left[\omega_{J-1}\right]} \\
{\left[\omega_{J}\right]}
\end{array}\right]
$$$$
e=\left[\begin{array}{c}
{\left[e_{1}\right]} \\
{\left[e_{2}\right]} \\
\vdots \\
{\left[e_{J-1}\right]} \\
{\left[e_{J}\right]}
\end{array}\right]
$$

The elements of the matrices are as follows:

$$
\left[A_{1}\right]=\left[\begin{array}{ccccccc}
0 & 0 & 0 & 1 & 0 & 0 & 0 \\
-\frac{h_{j}}{2} & 0 & 0 & 0 & -\frac{h_{j}}{2} & 0 & 0 \\
0 & -\frac{h_{j}}{2} & 0 & 0 & 0 & -\frac{h_{j}}{2} & 0 \\
0 & 0 & -\frac{h_{j}}{2} & 0 & 0 & 0 & -\frac{h_{j}}{2} \\
\left(a_{2}\right)_{1} & 0 & 0 & \left(a_{3}\right)_{1} & \left(a_{1}\right)_{1} & 0 & 0 \\
0 & \left(b_{10}\right)_{1} & \left(b_{2}\right)_{1} & \left(b_{3}\right)_{1} & 0 & \left(b_{9}\right)_{1} & \left(b_{1}\right)_{1} \\
0 & \left(c_{2}\right)_{1} & \left(c_{10}\right)_{1} & \left(c_{3}\right)_{1} & 0 & \left(c_{1}\right)_{1} & \left(c_{9}\right)_{1}
\end{array}\right],
$$




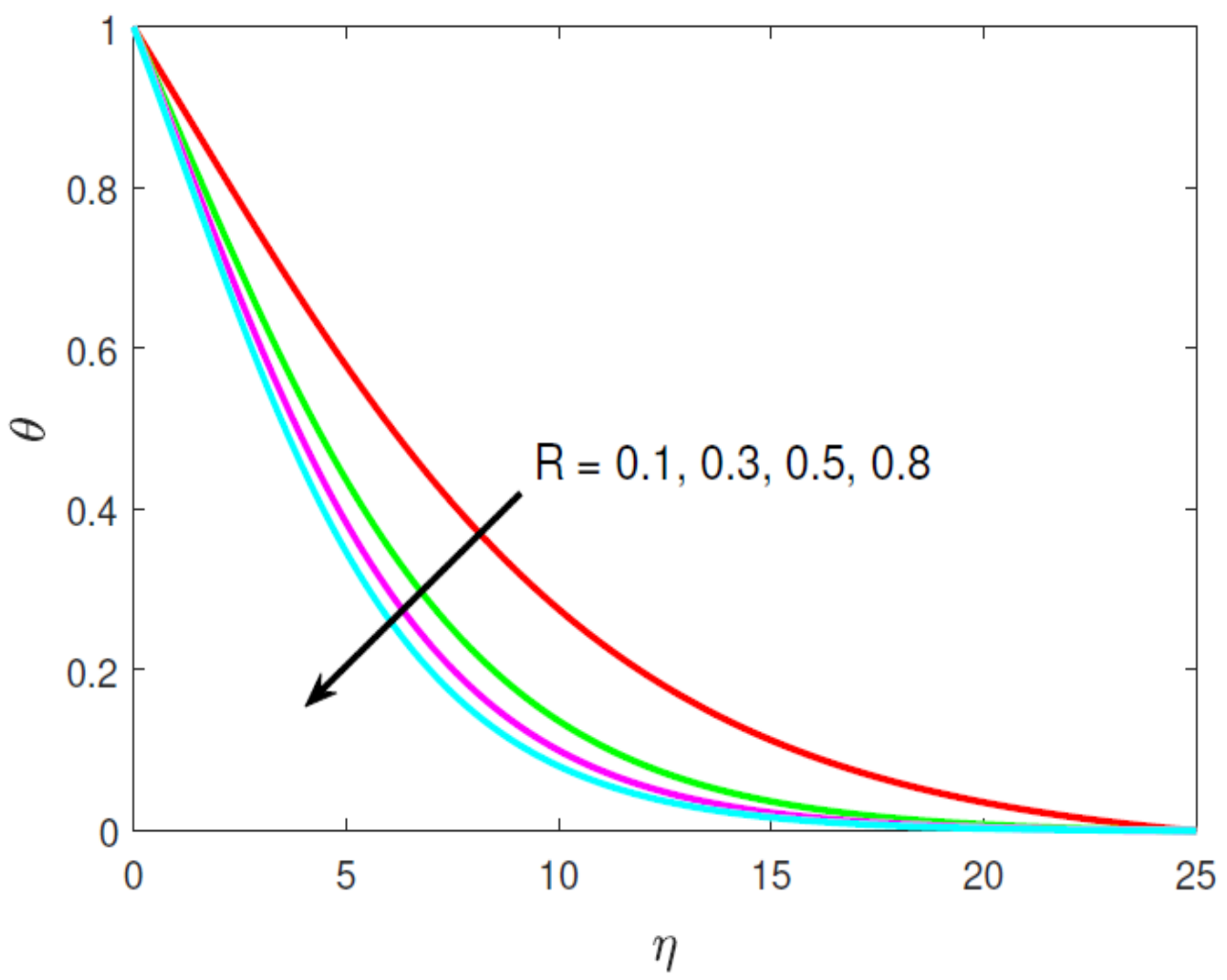

Figure 12. $\theta$ Variations for enhancing $R$.

$$
\begin{aligned}
{\left[A_{J}\right] } & =\left[\begin{array}{ccccccc}
-\frac{h_{j}}{2} & 0 & 0 & 1 & 0 & 0 & 0 \\
-1 & 0 & 0 & 0 & -\frac{h_{j}}{2} & 0 & 0 \\
0 & -1 & 0 & 0 & 0 & -\frac{h_{j}}{2} & 0 \\
0 & 0 & -1 & 0 & 0 & 0 & -\frac{h_{j}}{2} \\
\left(a_{6}\right)_{j} & 0 & \left(a_{8}\right)_{j} & \left(a_{3}\right)_{j} & \left(a_{1}\right)_{j} & 0 & 0 \\
\left(b_{6}\right)_{j} & 0 & \left(b_{8}\right)_{j} & \left(b_{3}\right)_{j} & 0 & \left(b_{9}\right)_{j} & \left(b_{1}\right)_{j} \\
\left(c_{6}\right)_{j} & \left(c_{8}\right)_{j} & 0 & \left(c_{3}\right)_{j} & 0 & \left(c_{1}\right)_{j} & \left(c_{9}\right)_{j}
\end{array}\right], 2 \leq j \leq J(57) \\
{\left[B_{J}\right] } & =\left[\begin{array}{ccccccc}
0 & 0 & 0 & -1 & 0 & 0 & 0 \\
0 & 0 & 0 & 0 & -\frac{h_{j}}{2} & 0 & 0 \\
0 & 0 & 0 & 0 & 0 & -\frac{h_{j}}{2} & 0 \\
0 & 0 & 0 & 0 & 0 & 0 & -\frac{h_{j}}{2} \\
0 & 0 & 0 & \left(a_{4}\right)_{j} & \left(a_{2}\right)_{j} & 0 & 0 \\
0 & 0 & 0 & \left(b_{4}\right)_{j} & 0 & \left(b_{10}\right)_{j} & \left(b_{2}\right)_{j} \\
0 & 0 & 0 & \left(c_{4}\right)_{j} & 0 & \left(c_{2}\right)_{j} & \left(c_{10}\right)_{j}
\end{array}\right], 2 \leq j \leq J
\end{aligned}
$$




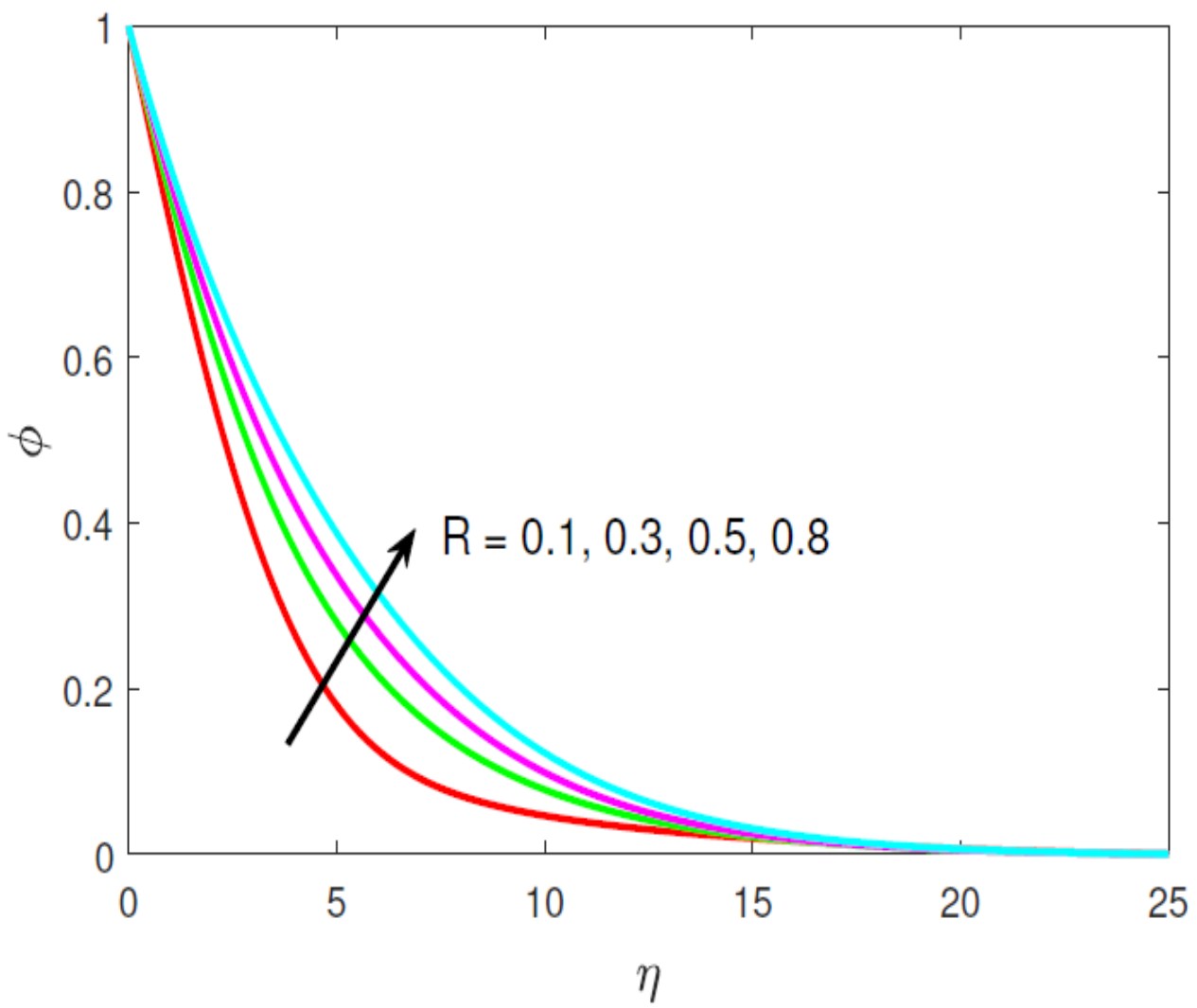

Figure 13. $\phi$ variations for enhancing $R$.

$$
\begin{aligned}
{\left[C_{J}\right] } & =\left[\begin{array}{ccccccc}
-\frac{h_{j}}{2} & 0 & 0 & 0 & 0 & 0 & 0 \\
1 & 0 & 0 & 0 & 0 & 0 & 0 \\
0 & 1 & 0 & 0 & 0 & 0 & 0 \\
0 & 0 & 1 & 0 & 0 & 0 & 0 \\
\left(a_{5}\right)_{j} & \left(a_{9}\right)_{j} & \left(a_{7}\right)_{j} & 0 & 0 & 0 & 0 \\
\left(b_{5}\right)_{j} & 0 & \left(b_{7}\right)_{j} & 0 & 0 & 0 & 0 \\
\left(c_{5}\right)_{j} & \left(c_{7}\right)_{j} & 0 & 0 & 0 & 0 & 0
\end{array}\right], 1 \leq j \leq J-1 \\
{\left[\omega_{1}\right] } & =\left[\begin{array}{c}
\omega v_{0} \\
\omega p_{0} \\
\omega t_{0} \\
\omega f_{1} \\
\omega v_{1} \\
\omega p_{1} \\
\omega t_{1}
\end{array}\right],
\end{aligned}
$$




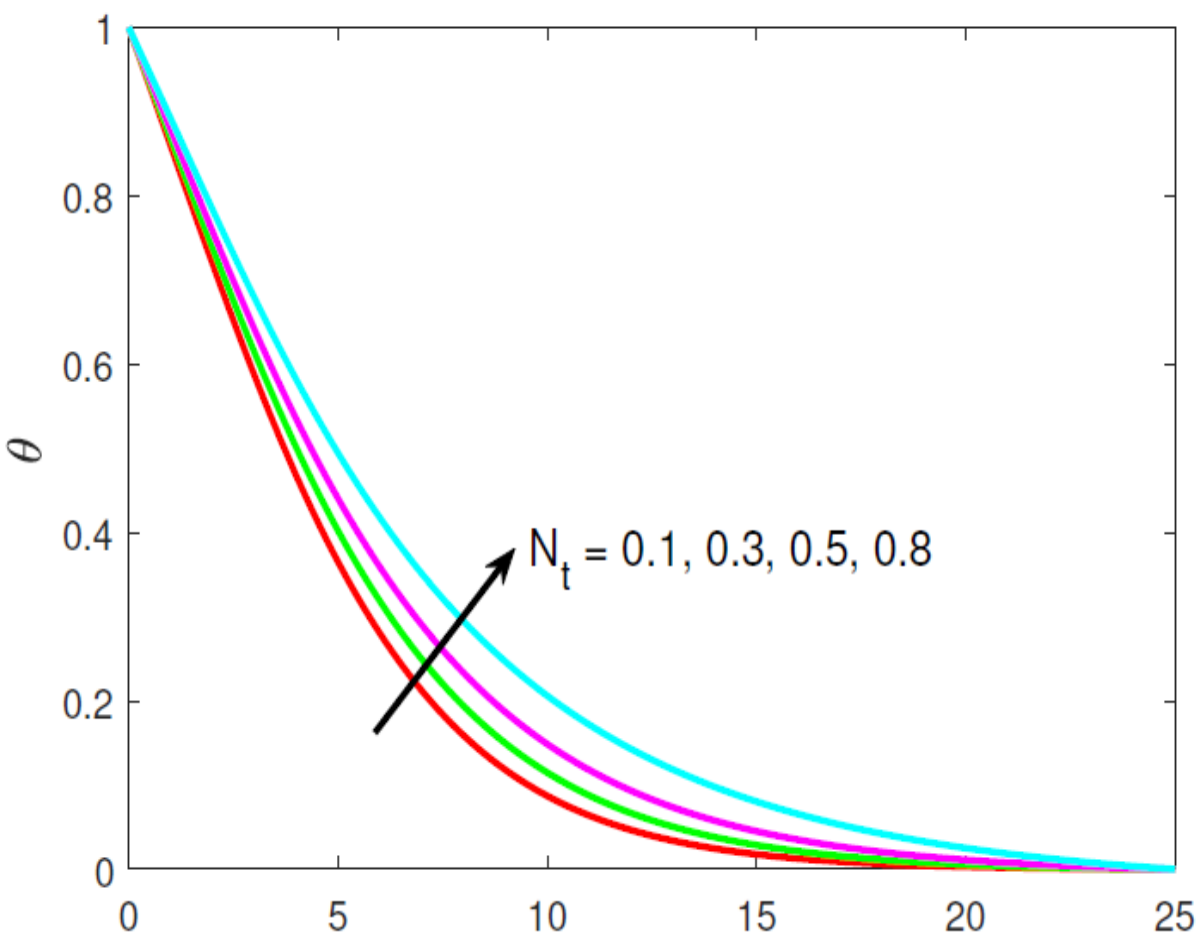

Figure 14. $\theta$ Variations for enhancing $N_{t}$.

$$
\text { and }\left[e_{j}\right]=\left[\begin{array}{c}
\left(e_{1}\right)_{j-(1 / 2)} \\
\left(e_{2}\right)_{j-(1 / 2)} \\
\left(e_{3}\right)_{j-(1 / 2)} \\
\left(e_{4}\right)_{j-(1 / 2)} \\
\left(e_{5}\right)_{j-(1 / 2)} \\
\left(e_{6}\right)_{j-(1 / 2)} \\
\left(e_{7}\right)_{j-(1 / 2)}
\end{array}\right], 1 \leq j \leq J
$$

Equation (53) is solved by using the LU (lower-upper) decomposition method. From this decomposition matrix method, the solution is obtained and the procedure is remained till the convergence criterion is satisfied and the process is stopped when $\omega v_{0}^{i} \leq 0.001$. In this problem, the extreme $\xi$ and $\eta$ values are in order considered as 1 and 25. Table 1 shows the calculations with various grid sizes $(\Delta \eta)$. It noticed that the step size $(\Delta \eta=0.005)$ gives accurate results compared with other step sizes for this problem. Therefore the step size is fastened as $(\Delta \eta=0.005)$ to obtain the solution of this problem and $(20 \times 500)$ meshes are consider in the domain. Table 2 exhibits the order of accuracy of the numerical scheme. To raise the accurateness of the solution, the convergence criterion has been fixed as $10^{-5}$ at all grid points. Fig. 2 illustrates the solution procedure of Keller box method, boundary layer mesh and Keller box element. 
Table 1. A grid independence study

\begin{tabular}{|c|c|c|}
\hline$\Delta \eta$ & $N u_{x}$ & $S h_{x}$ \\
\hline 0.25 & 0.789819 & 0.679725 \\
\hline 0.05 & 0.783485 & 0.678332 \\
\hline 0.025 & 0.783443 & 0.678327 \\
\hline 0.01 & 0.783429 & 0.678315 \\
\hline 0.05 & 0.783421 & 0.678311 \\
\hline
\end{tabular}

Table 2. The order of accuracy for the numerical scheme

\begin{tabular}{|c|c|c|}
\hline & $N u_{x}$ & $S h_{x}$ \\
\hline $10^{-1}$ & 0.79335 & 0.67791 \\
\hline $10^{-2}$ & 0.77292 & 0.66805 \\
\hline $10^{-3}$ & 0.77196 & 0.67889 \\
\hline $10^{-4}$ & 0.77145 & 0.67862 \\
\hline $10^{-5}$ & 0.77188 & 0.67879 \\
\hline $10^{-6}$ & 0.77187 & 0.67872 \\
\hline
\end{tabular}

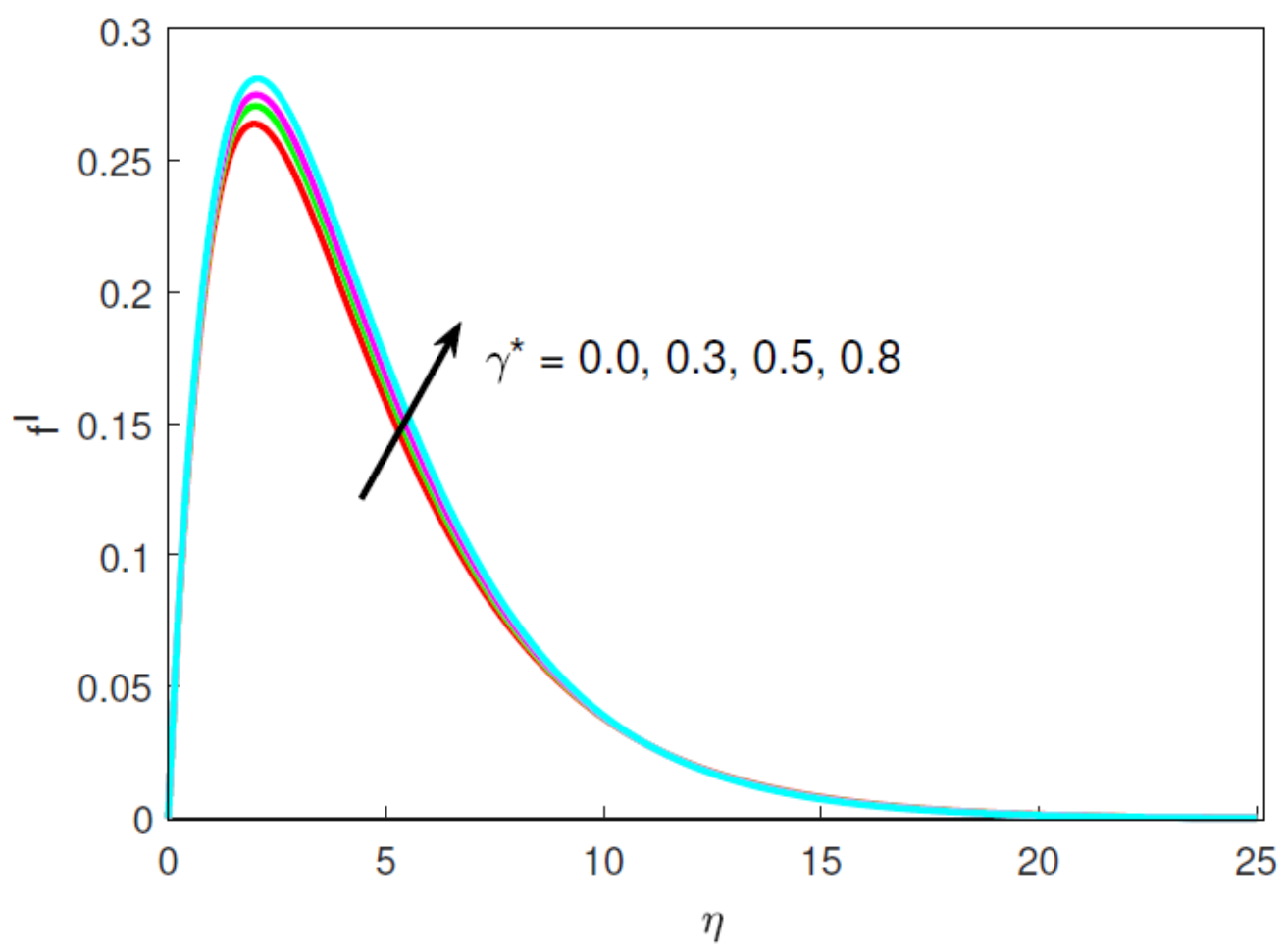

Figure 15. $f^{\prime}$ variations for enhancing $\gamma^{*}$.

\section{Validation of Numerical Scheme}

The accurateness of the results computed using KBM is compared with previously published results available in literature. Table 3 shows the KBM solutions for local heat 


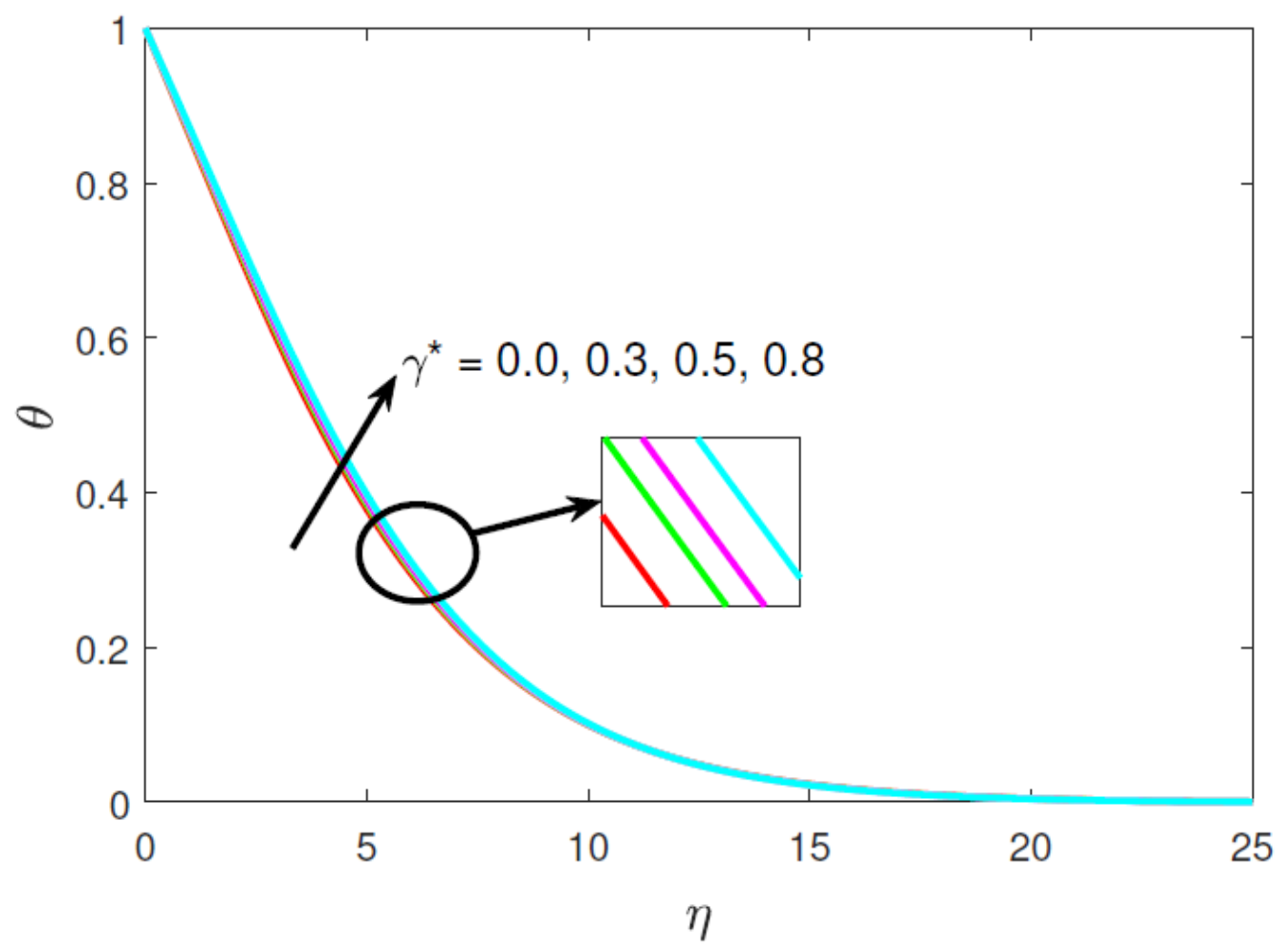

Figure 16. $\theta$ Variations for enhancing $\gamma^{*}$.

transfer rate for several $\xi$ values which are compared with the computations of Merkin [32] and Prasad et al. [58] in the absence of Powell Eyring fluid, nanofluid, variable temperature parameter, chemical reaction, Schmidt number and thermal radiation. Scrutiny of the table confirms that generally worthy agreement is attained. This correlation testifies to the validity of the KBM code and confidence in the accuracy is justifiably high. It further confirms that for Newtonian fluid hydromagnetic boundary layer flow external to a cylinder under dual buoyancy, the Nusselt number is depleted with increasing distance beside the curvy surface i.e. lower value of the stagnation point $(\xi=0)$ implying higher heat transfer rate.

\section{Results and discussion}

A comprehensive mathematical examination is conducted to scrutinize the key emerging parameters on transport characteristics of the fluid. Figures 3-20 elucidate the variations in momentum, heat and nanoparticle concentration characteristics and consistently smooth profiles are achieved in the free stream. The KBM simulations are worked out by considering adequate value to the infinite boundary condition. The characteristics of local skin friction factor, heat transfer rate and mass transfer rate are displayed in Figs. 21-30 and Table 4. The default values of pertinent parameters in present computation 


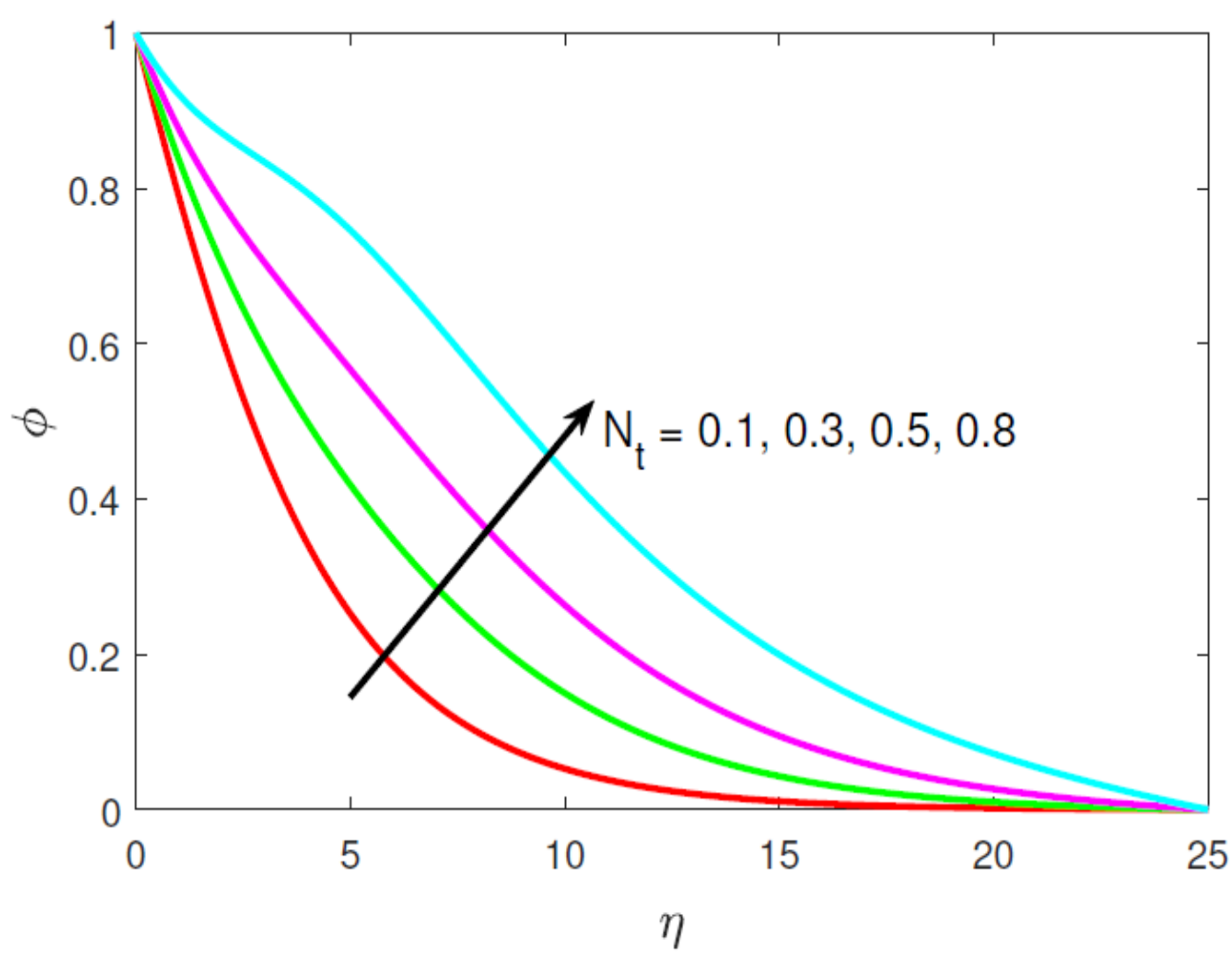

Figure 17. $\phi$ Variations for enhancing $N_{t}$.

Table 3. Validation of current results with Merkin [32] and Prasad et al. [58] for local rate of heat transfer against varies $\xi$ values when $D a \rightarrow \infty, R \rightarrow \infty$, $\wedge=N_{t}=N_{b}=\gamma^{*}=\varepsilon=\varepsilon_{1}=0, S c=0.6, H a=0.5, P_{r}=0.71$. (note infinite Da and $\mathrm{R}$ imply vanishing porous medium fibres and absence of radiative heat transfer).

\begin{tabular}{|c|c|c|c|}
\hline Parameter & \multicolumn{3}{|c|}{$N u_{x}$} \\
\hline$\xi$ & Merkin [32] & Prasad et. al[58] & Present \\
\hline 0.0 & 0.4212 & 0.4211 & 0.4213 \\
\hline 0.2 & 0.4204 & 0.4206 & 0.4208 \\
\hline 0.4 & 0.4182 & 0.4185 & 0.4187 \\
\hline 0.6 & 0.4145 & 0.4146 & 0.4147 \\
\hline 0.8 & 0.4093 & 0.4095 & 0.4096 \\
\hline 1.0 & 0.4025 & 0.4027 & 0.4028 \\
\hline
\end{tabular}

are considered as $\mathrm{Ha}=0.5, \mathrm{~N}=0.5, \wedge=0.5, \gamma^{*}=0.2, \varepsilon_{1}=0.3, \varepsilon=0.2, R=0.2$, $D a=0.5, N_{t}=0.2, N_{b}=0.3, P_{r}=0.71, K r=0.5$, and $S c=0.3$. This data is based on physically viable material properties for magnetic coatings, and has been extracted from multiple sources including Guiseppi-Elie [33] (rheological parameters), Prakash et al. [34] (nanoscale and species diffusion parameters), Mansour and Viskanta [35] (radiative and convective parameters) and Hughes and Young [36] (magnetic parameters) and Umavathi and Beg [37] (porous Darcy and Forchheimer parameters). 


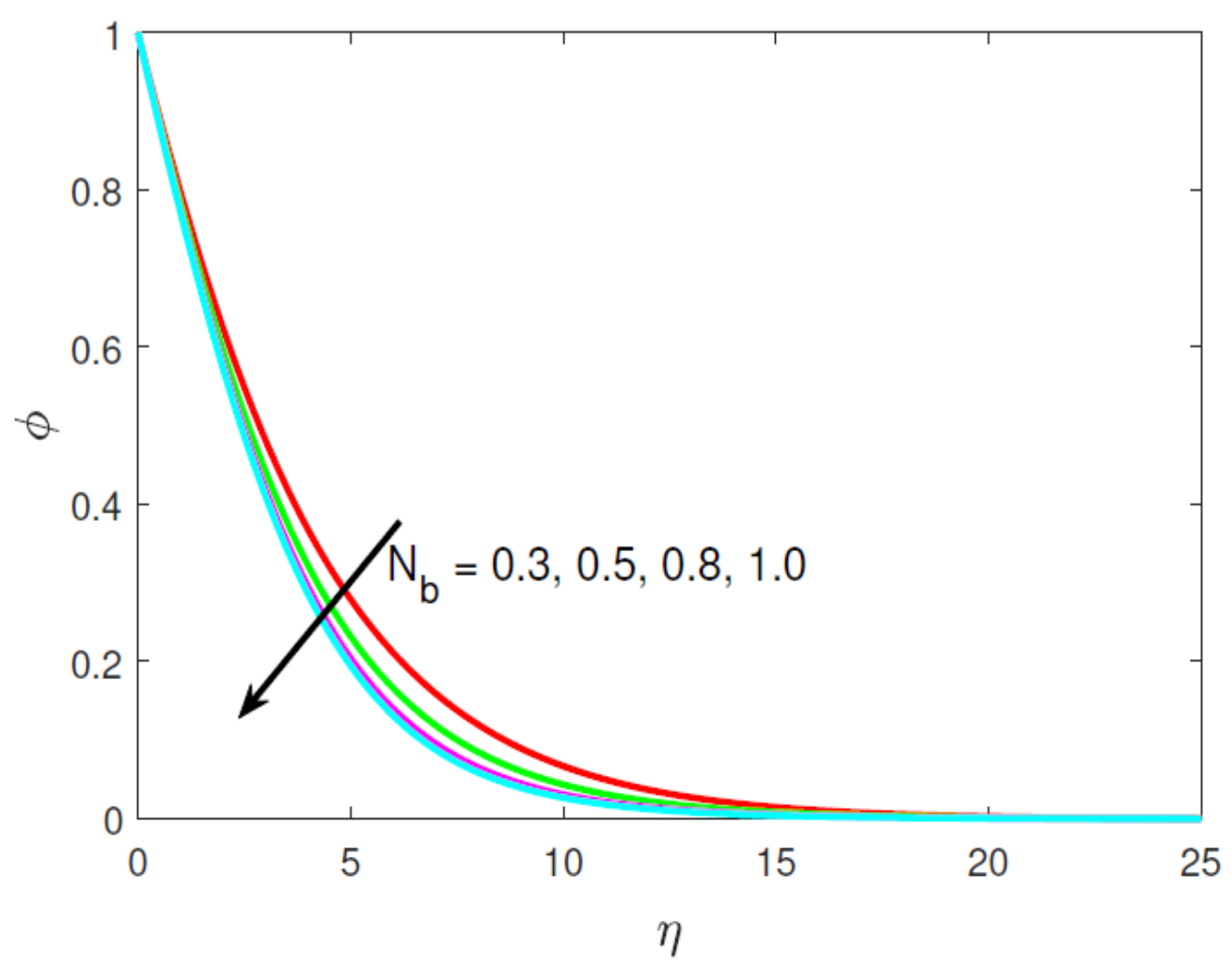

Figure 18. $\phi$ Variations for enhancing $N_{b}$.

Figures 3-5 exemplify the characteristics of fluid flow, heat transfer and nanoparticle concentration distributions for the variations in magnetic field parameter $(\mathrm{Ha})$. In the electrically conducting fluid (e.g. magnetic nano-polymer), the radial direction experiences the imposed magnetic field $\left(B_{0}\right)$ and this generates a transverse (orthogonal) hydromagnetic retarding force (Lorentz force) which acts along the cylinder longitudinal axis and resists the boundary layer motion. The magnitude of Lorentz force increases while the magnetic field strength $\left(B_{0}\right)$ is magnified, and this inhibits momentum development and significantly reduces the nanofluid velocity (Fig. 3). Hence, Momentum (hydrodynamic) boundary layer thickness diminishes for amplifying the magnetic field. The implication is that the deposition of the coating magnetic nano-polymer can be more effectively controlled via a boost in radial magnetic field which permits more homogenous distribution of the nano-polymer over the cylinder periphery and more even thickness of the coating, as noted by Khan et al. [38], Yu et al. [39], Ganesan et al. [40] and Wang et al. [41]. The classical velocity overshoot arising near the wall (cylinder surface) is also clearly computed and is systematically suppressed with greater values of $H a$. Maximum acceleration and largest hydrodynamic boundary layer thickness corresponds to the electrically non-conducting case $(H a=0)$. Although there is a weak 


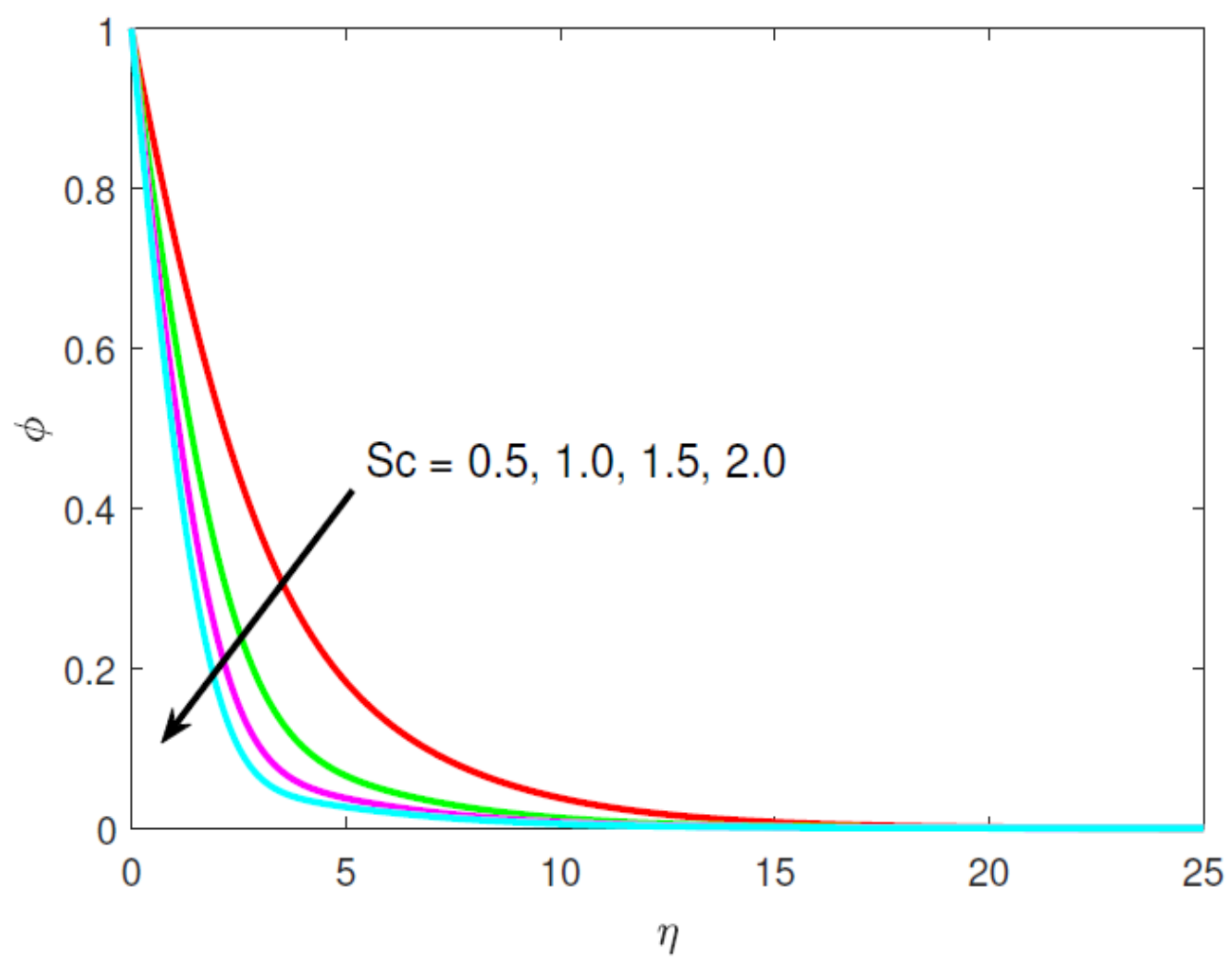

Figure 19. $\phi$ Variations for enhancing $S c$.

transition in this behaviour closer to the free stream, the principal magnetic field impact on velocity distribution is flow inhibition, although reversal in the flow is not observed even at highest magnetic field $(H a=1.5)$. The evolutions in temperature and nanoparticle concentration with magnetic field are displayed in Figs. 4 and 5, respectively. Strengthening the magnetic field results in enhancing the Lorentz force which necessitates greater work expenditure by nanofluid in dragging against the magnetic field action. Thermal energy is generated due to this excess work which strongly heats the coating regime and raises the fluid heat transfer. A consistent monotonous decline in temperatures from the cylindrical surface to the free stream is computed (Fig. 4). Simultaneously the nanoparticle diffusion is aided in the boundary layer i.e. nanoparticle concentration (Fig. 5) and the related nanoparticle concentration boundary layer thickness are boosted. Magnetic field therefore has the double advantage of flow regulation and enforcement of a more homogenous migration of nanoparticles; however, it leads to temperature elevation. The response in concentration is also consistent at all values of transverse coordinate.

Figures 6-7 illustrate the impact of the first Powell-Eyring rheological parameter, $\varepsilon\left(=\frac{1}{\mu_{f} \beta_{1} b}\right)$, on velocity and temperature distributions, respectively. The fluid flow and heat transfer distributions and the related boundary layers thicknesses are generally 


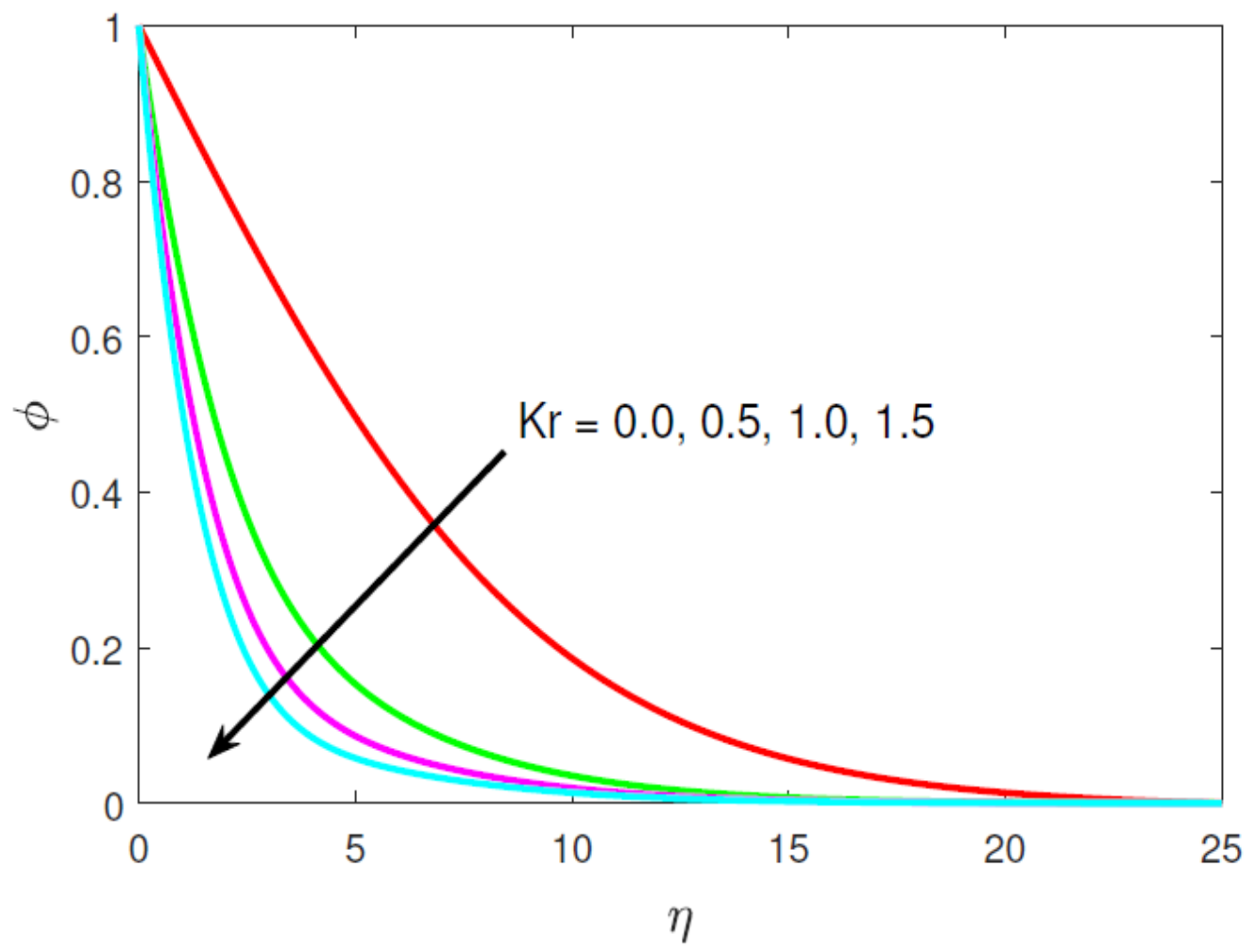

Figure 20. $\phi$ Variations for enhancing $K r$.

enhanced for increasing $\varepsilon$. Generally, higher fluid parameter $\varepsilon$ values results in reducing the fluid dynamic viscosity $\left(\mu_{f}\right)$. The first Powell-Eyring rheological parameter features in the augmented shear term, $\left[1+\varepsilon\left(1-\varepsilon_{1} \xi^{2} f^{\prime \prime 2}\right)\right] f^{\prime \prime \prime}$, in the momentum boundary layer Eqn. (11). Although absent in temperature distribution Eqn. (12), the velocity and heat transfer fields coupling terms i.e. $f \theta^{\prime}$ and $\xi\left[\frac{\partial \theta}{\partial \xi} f^{\prime}-\frac{\partial f}{\partial \xi} \theta^{\prime}\right]$, will inevitably result in a slight modification in temperature. Though primarily there is a weak reduction in fluid flow (Fig. 6) with greater $\varepsilon$ values, this is superseded with a clear boost in velocity magnitudes further from the wall which is sustained into the free stream. It is observed that the temperature elevation is sustained at all locations, transverse to the cylinder surface (Fig. 7). Newtonian nanofluid $(\varepsilon=0)$ clearly produces the lowest temperature magnitudes. Therefore, a Newtonian model under-predicts temperatures in the magnetic nanocoating. A more realistic estimate is therefore only possible with a non-Newtonian rheological model.

Figures 8 and 9 show the influence of the second Powell-Eyring rheological parameter, $\varepsilon_{1}\left(=\frac{\nu_{f}^{2}}{2 b^{2} a^{4}}\right)$, on fluid flow and heat transfer profiles, respectively. This parameter occurs both in the shear term in Eqn. (11) (as noted earlier) and in the amplified thermal diffusion term, $\varepsilon_{1}\left(\theta^{\prime}\right)^{2}$, in the thermal boundary layer Eqn. (12). Distinct from the first rheological term, this term is proportional to the square of dynamic viscosity 


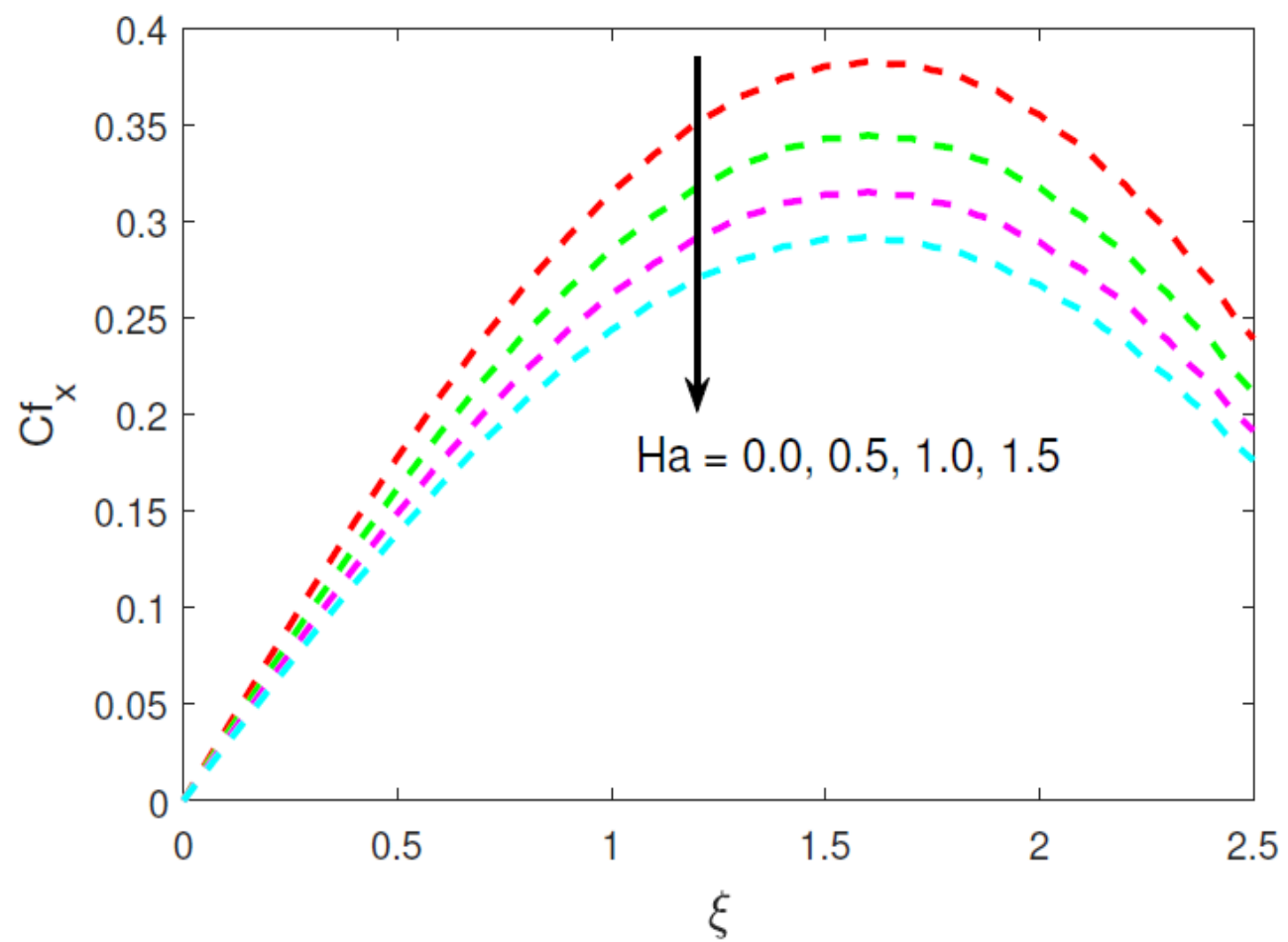

Figure 21. $C f_{x}$ Variations for enhancing $H a$.

(and consequently the constant density of magnetic coating fluid, as considered here, is proportional to the square of dynamic viscosity). The other parameters, $b$ and $a$ are fixed (constant material parameter and cylinder radius). A significant increment in $\varepsilon_{1}$ induces a very weak decrease in both velocity and temperature distributions. The impact of this effect is lower than the first Powell-Eyring rheological parameter which is probably attributable to the coupling of the higher order derivative, $f^{\prime \prime \prime}$, to the first rheological parameter and the absence in the second parameter. The upshot is that a trivial deceleration in the fluid flow and a slight reduction in heat transfer are produced with greater $\varepsilon_{1}$. Ishaq et al. [42] has obtained the similar trend.

Figure 10 indicates that elevating Darcy parameter reduces the velocity, $f^{\prime}$, significantly. The Darcian force, $\frac{-f^{\prime}}{D a}$, which is a drag force occurs in momentum Eqn. (11). It is well known that the flow will be decelerated with increasing Darcy number, $D a=\left(\frac{K \sqrt{G r}}{a^{2}}\right)$. With increasing Darcy number, there is a notable solid material fibers decrease in porous media. This reduces the available surface area for conduction heat transfer and effectively cools the regime. In other words a low permeability (low Darcy number) regime achieves greater conduction heat transfer due to larger surface area of fibers for heat transmission and a high permeability (high Darcy number) induces the opposite response. Higher Darcy number values notably deplete the heat transfer and these results concur closely with the findings of Vafai and Tien [43].

Figure 11 presents the velocity profiles for increasing buoyancy ratio parameter $(N)$ 


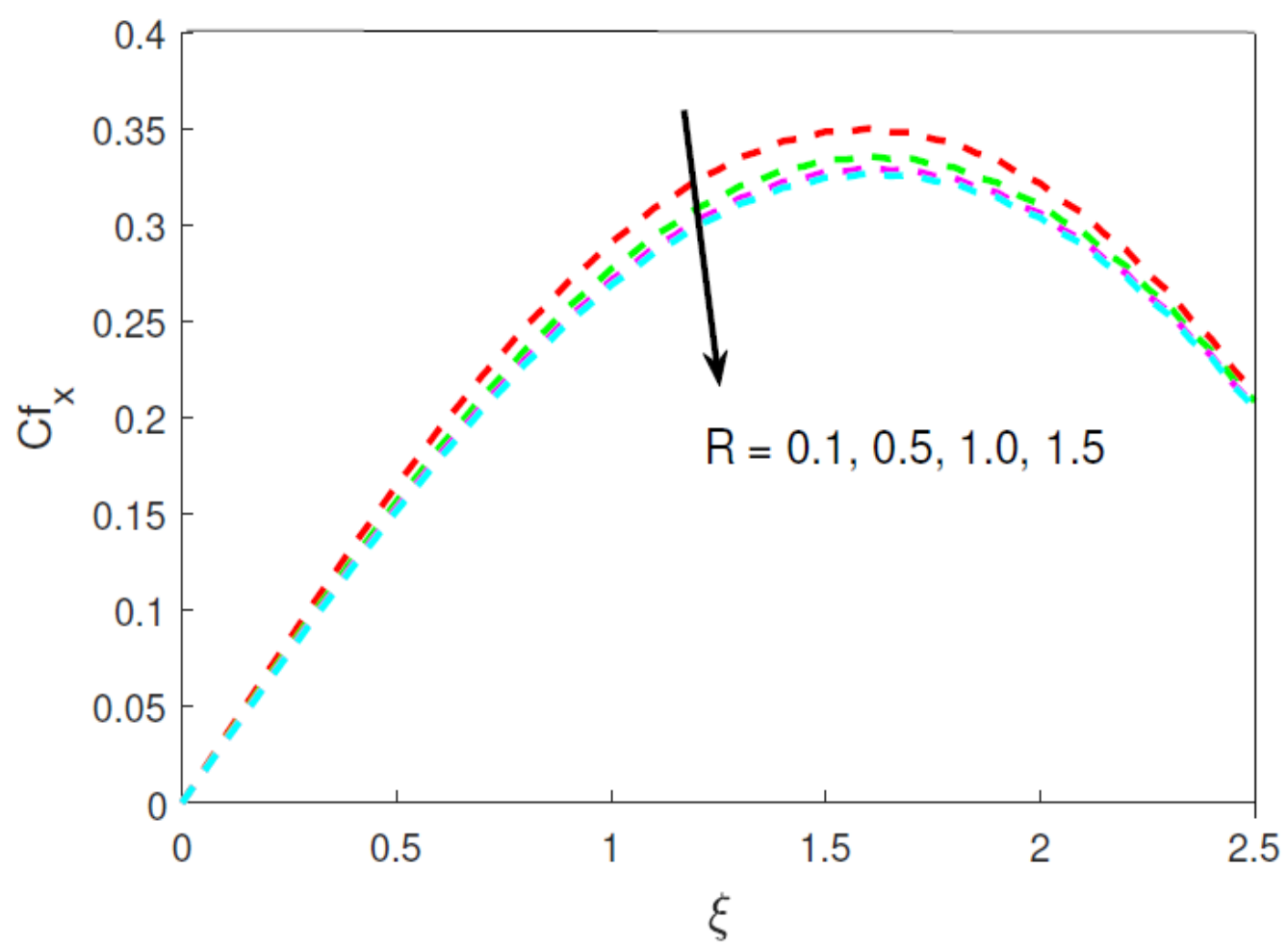

Figure 22. $C f_{x}$ Variations for enhancing $R$.

values. Buoyancy ratio parameter exemplifies the ratio of solutal buoyancy to the thermal buoyancy force. The Buoyancy ratio parameter can also be viewed as the coupling parameter between the momentum Eqn. (11) and the nanoparticle concentration Eqn. (13), i.e. $\frac{\sin \xi}{\xi}(\theta-N \phi)$, as noted by Jaluria and Gebhart [44]. It is observed that the flow is retarded markedly i.e. velocity suppressed with increasing $N$ values (note for the case $N=0$, the species buoyancy is negated as well as the fluid flow and nanoparticle concentration boundary layer equations are de-coupled; furthermore for $N<1$ the species buoyancy is dominated by the thermal buoyancy effect). Therefore, amplifying buoyancy ratio parameter decelerates the fluid flow.

Figure 12 elucidates heat transfer variations owing to changes in radiative heat flux. With the thermal conductivity variation parameter, the Rossleand conduction-radiation parameter $\left(R=\frac{k_{0} k_{a}}{4 \sigma_{B} T_{\infty}^{3}}\right)$ is also an additional feature in the thermal diffusion term, $\left(\frac{4 \theta^{\prime \prime}}{3 P_{r} R}\right)$. Although the parameter is in the denominator, the contribution is still that of conduction heat transfer relative to radiation heat transfer. As $R$ is increased thermal conduction becomes progressively larger (for $R<1$ it always dominates thermal radiation) and this leads to suppression in heat transfer profiles. For lower $R$ values, the loss of thermal radiative heat transfer is less and this energizes the nanofluid which helps to maintain high temperatures. It is noteworthy that the Rosseland model assumes that radiative evenness is sustained in the simulations and the nanofluid is gray. Furthermore, 


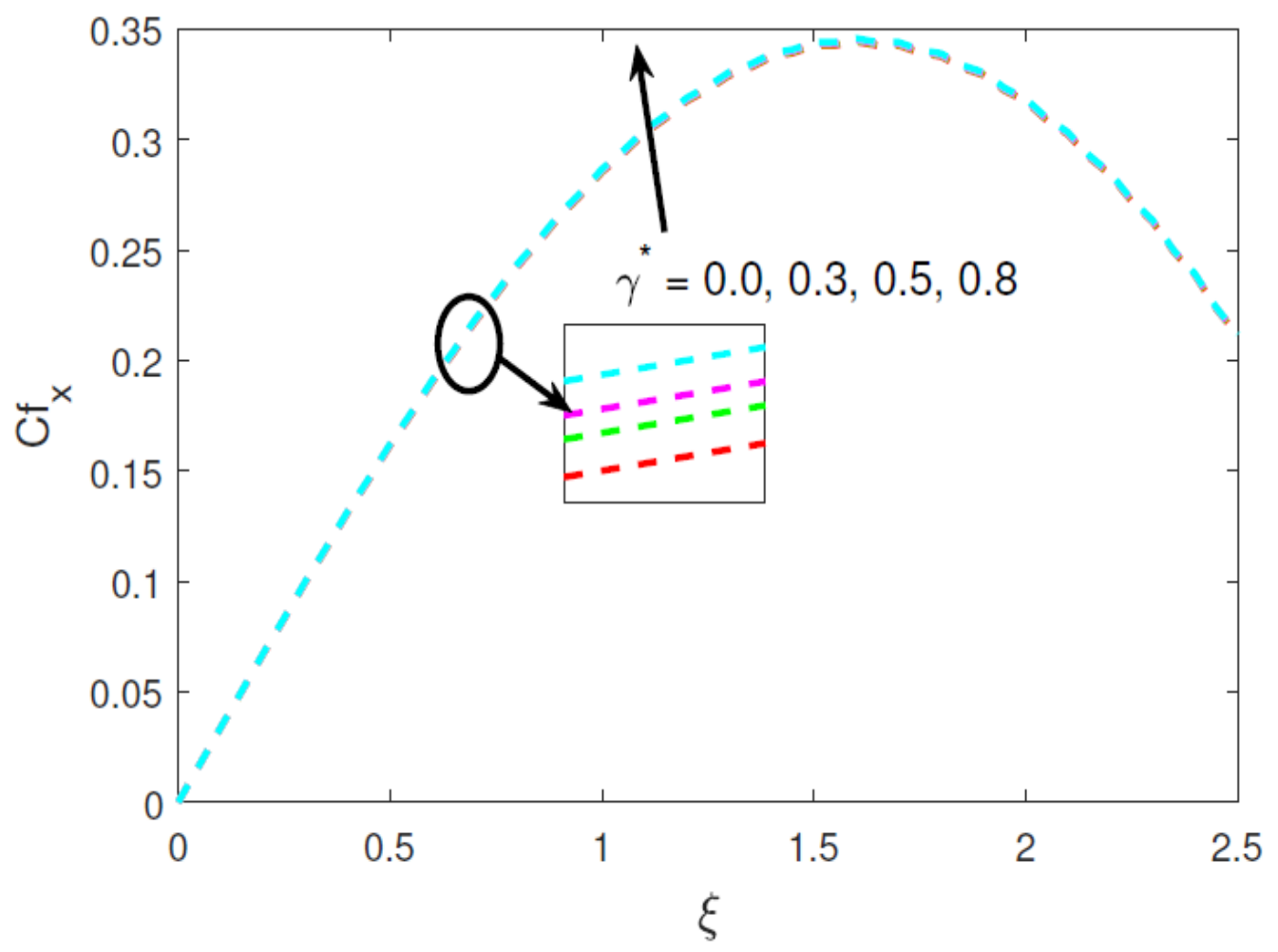

Figure 23. $C f_{x}$ Variations for enhancing $\gamma^{*}$.

the intensity is the black-body intensity at the nanofluid temperature. In this flux approximation, the requirement is that the optical thickness needs to exceed 3 for reasonable accuracy as noted by Modest [45] and later Beg et al. [46]. The considered nanofluid medium decelerates the passage of radiative heat flux due to the impact of the dimensionless quantifications such as optical thickness and absorption coefficient. While optical thickness is unity thermal radiation intensity drops by an exponential factor. It is to be noted that propagation distance, medium density, and absorption coefficient are the important components to control the optical thickness. Though the flux model is much simpler than other algebraic approximations (e.g. Hamaker six-flux model), it does predict the influence of radiative flux reasonably well. The results concur with $\mathrm{Lu}$ et al. [47]. Figure 13 elucidates the thermal radiation parameter consequences on mass transfer distribution. It is perceived that mass transfer $(\phi)$ upsurges for higher thermal radiation parameter values. A reduction in radiative heat flux (higher $R$ values) encourages the diffusion of nanoparticles and enhances nanoparticle concentration boundary layer thickness. Similar patterns have been reported in other studies for radiative nanofluid flow from curved geometries including Makinde and Animasaun [20].

Figure 14 visualizes the temperature distribution characteristics for enhancing thermophoresis parameter $\left(N_{t}\right)$ values. In thermophoresis, the heated nanoparticles are pushed from a hot surface to a cold area under a temperature gradient. Thermophoretic body force therefore mobilizes nanoparticle migration from the cylinder surface, and also 


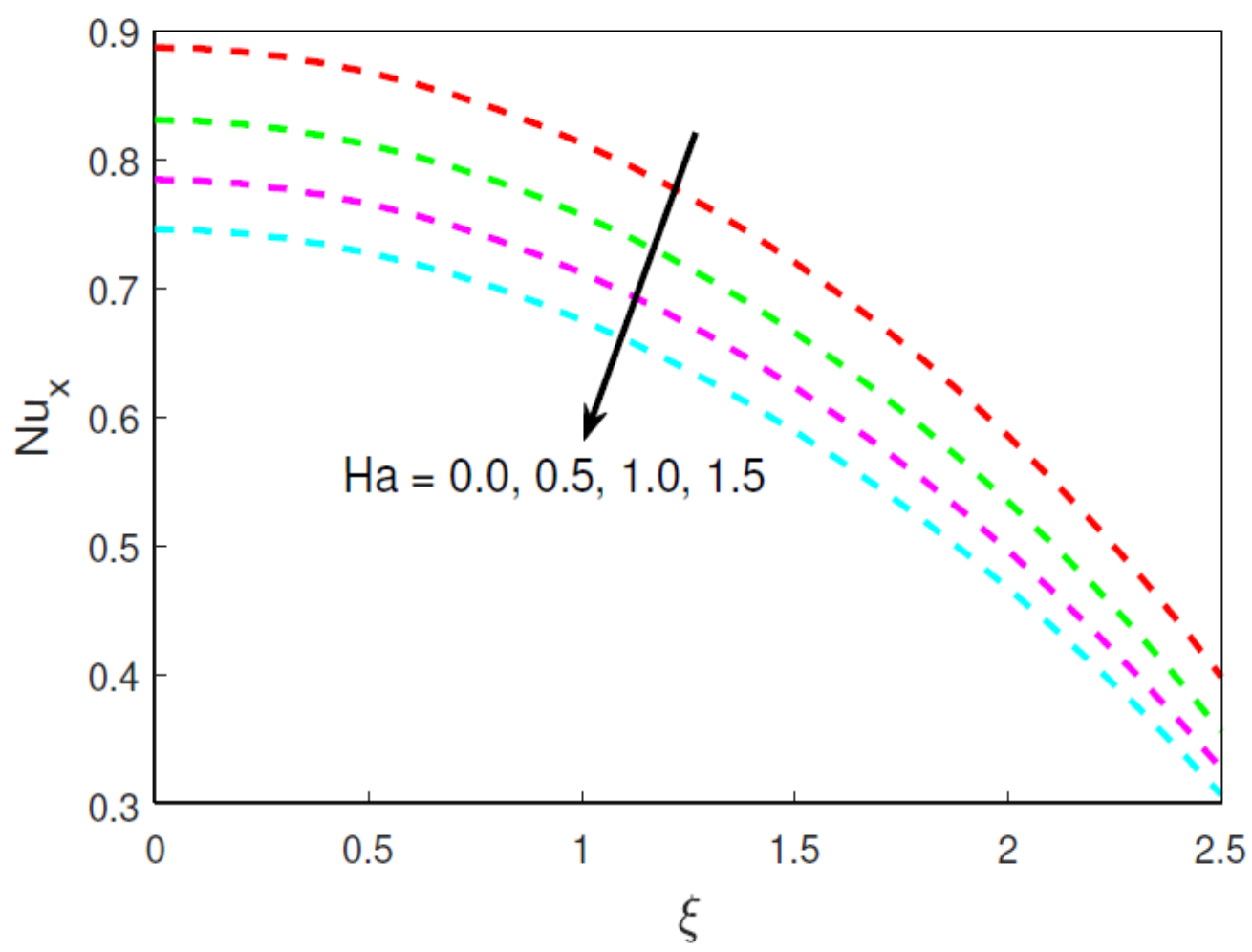

Figure 24. $N u_{x}$ Variations for enhancing $H a$.

encourages heat diffusion into the boundary layer away from the wall. This causes an elevation in nanofluid temperature and an accompanying increment in the heat transfer profiles.

Figures 15 and 16 in order depict the fluid momentum and energy transfer distributions features for amplifying thermal conductivity variation parameter $\left(\gamma^{*}\right)$ values. It is seen that both characteristics exhibit a slight elevation with larger $\gamma^{*}$ values. Thermal conductivity variation parameter features in amplified thermal diffusion term, $\frac{\gamma^{*} \theta \theta^{\prime \prime}}{P_{r}}$, in heat transfer Eqn. (12) and accentuates heat transfer inside the nanofluid. A greater nanofluid thermal conductivity intensifies conduction heat transfer which assists also in thermal diffusion and momentum diffusion. This results in a distinct fluid momentum and energy transfer escalation. Evidently inclusion of thermal conductivity variation produces results which more accurately predict the velocity and temperature magnitudes. Absence of this parameter $\gamma^{*}=0$ leads to an under-prediction in both quantities and lower momentum and lower thermal boundary layer thickness estimates, which are undesirable in manufacturing operations and can incur expenses, as noted by Jaluria [48].

Figure 17 displays the mass transfer characteristics for magnifying thermophoresis parameter $\left(N_{t}\right)$. It is confirmed that thermophoretic body force promotes the transport of nanoparticles away from the heated albeit isothermal cylinder wall into the nanofluid 


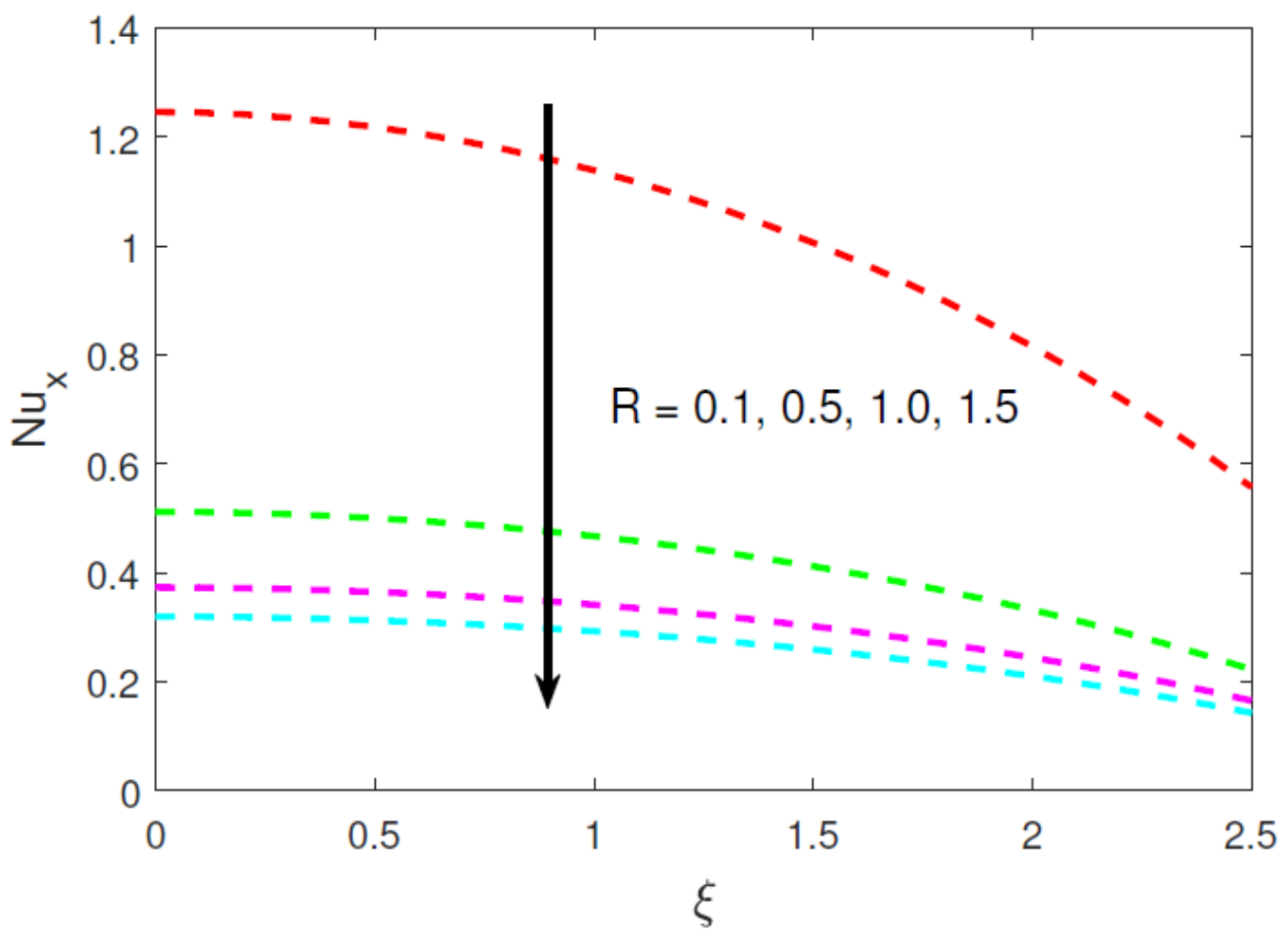

Figure 25. $N u_{x}$ Variations for enhancing $R$.

boundary layer regime, and therefore enhances nanoparticle concentration magnitudes. The amplification in magnitudes is considerably greater than temperatures since thermophoresis is essentially a species diffusion phenomenon which affects thermal field, as simulated in the quadratic temperature derivative term, $\left(N_{t}\left(\theta^{\prime}\right)^{2}\right)$ in heat transfer Eqn. (12). A notable mass transfer augmentation is induced due to the presence of the term, $\left(N_{t} \theta^{\prime \prime}\right)$, in mass transfer Eqn. (13).

Figure 18 displays mass transfer profiles for amplifying Brownian motion parameter $\left(N_{b}\right)$ values. It is known that higher $N_{b}$ values (in which the Buongiorno model [49] correspond to smaller nanoparticle sizes and a reduction in nanoparticle ballistic collisions) diminish the nanoparticle concentration i.e. there is a depletion in the volume fraction (percentage doping of the base fluid with nanoparticles). In the Buongiorno model, $N_{b}$ arises in a coupled thermal-species diffusion term, in heat transfer Eqn. (12), through $N_{b} \theta^{\prime} \phi^{\prime}$. When this term is magnified, the species diffusion is reduced. A limitation of this model is that actual nanoparticle types e.g. metallic oxides, carbon nanotubes (CNTs) or carbon silicates cannot be simulated since a framework for their properties can not be accommodated. This is achievable in the Tiwari-Das model, as noted by Prakash et al. [34]. However, the Tiwari-Das model does not feature a mechanism for species diffusion since it omits a concentration balance equation. A possible remedy to this dilemma is the fusion of both models and this is currently under investigation.

Figure 19 presents the mass transfer features for magnifying Schmidt number. This 


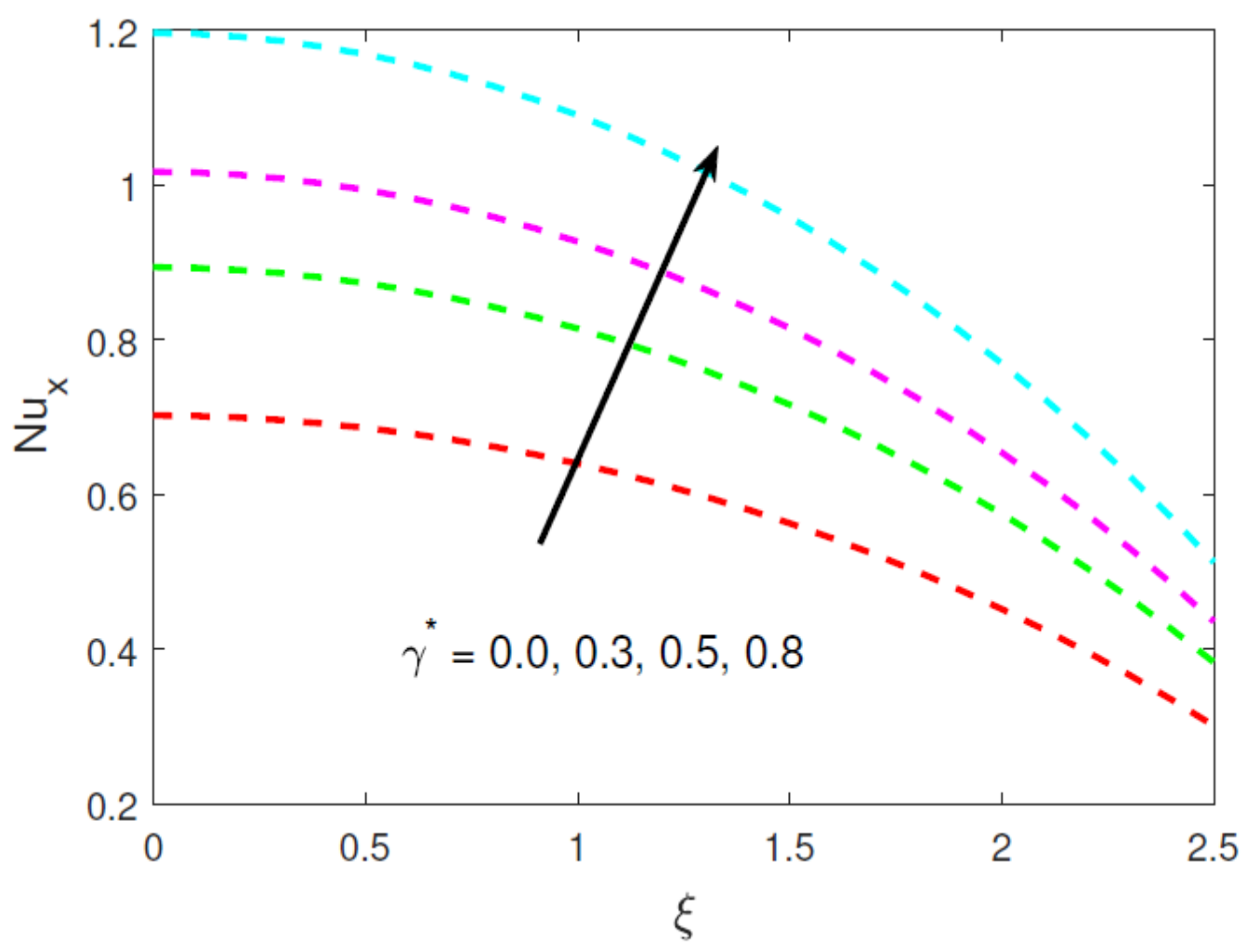

Figure 26. $N u_{x}$ Variations for enhancing $\gamma^{*}$.

reveals that mass transfer distribution decreases for enhancing Schmidt number values. In general, higher Schmidt number values decline the molecular diffusivity of nanoparticles in the base fluid; consequently the concentration profiles falls down. Figure 20 elucidates the mass transfer features for enhancing chemical reaction parameter $(K r)$. The present analysis experiences the destructive chemical reaction because higher chemical reaction parameter values diminish nanoparticle concentration boundary layer thickness. This elucidates that chemical reaction variation can control the mass diffusion rates. A homogenous first order chemical reaction has been considered and modelled with the term, $-K r \phi$, in the nanoparticle species diffusion Eqn. (13). The negative nature of this term indicates destructive chemical reaction and clearly its augmentation will dampen the nanoparticle concentration values as greater conversion of original species to a new product via chemical reaction will be induced with greater $(K r)$ values. Similar trends in magnetohydrodynamic boundary layer thermo solutal flows are presented by Sivaraj and Kumar [50, 51], Kumar and Sivaraj [52], Sheri et al. [53] and Beg et al. [54]. Of course, extra complex chemical reactions may take place e.g. heterogenous reactions, and these may be addressed in future studies.

Figures 21 and 22 show the skin friction coefficient $\left(C f_{x}\right)$ variations for amplifying magnetic field parameter $(H a)$ and thermal radiation parameter $(R)$, respectively. This 


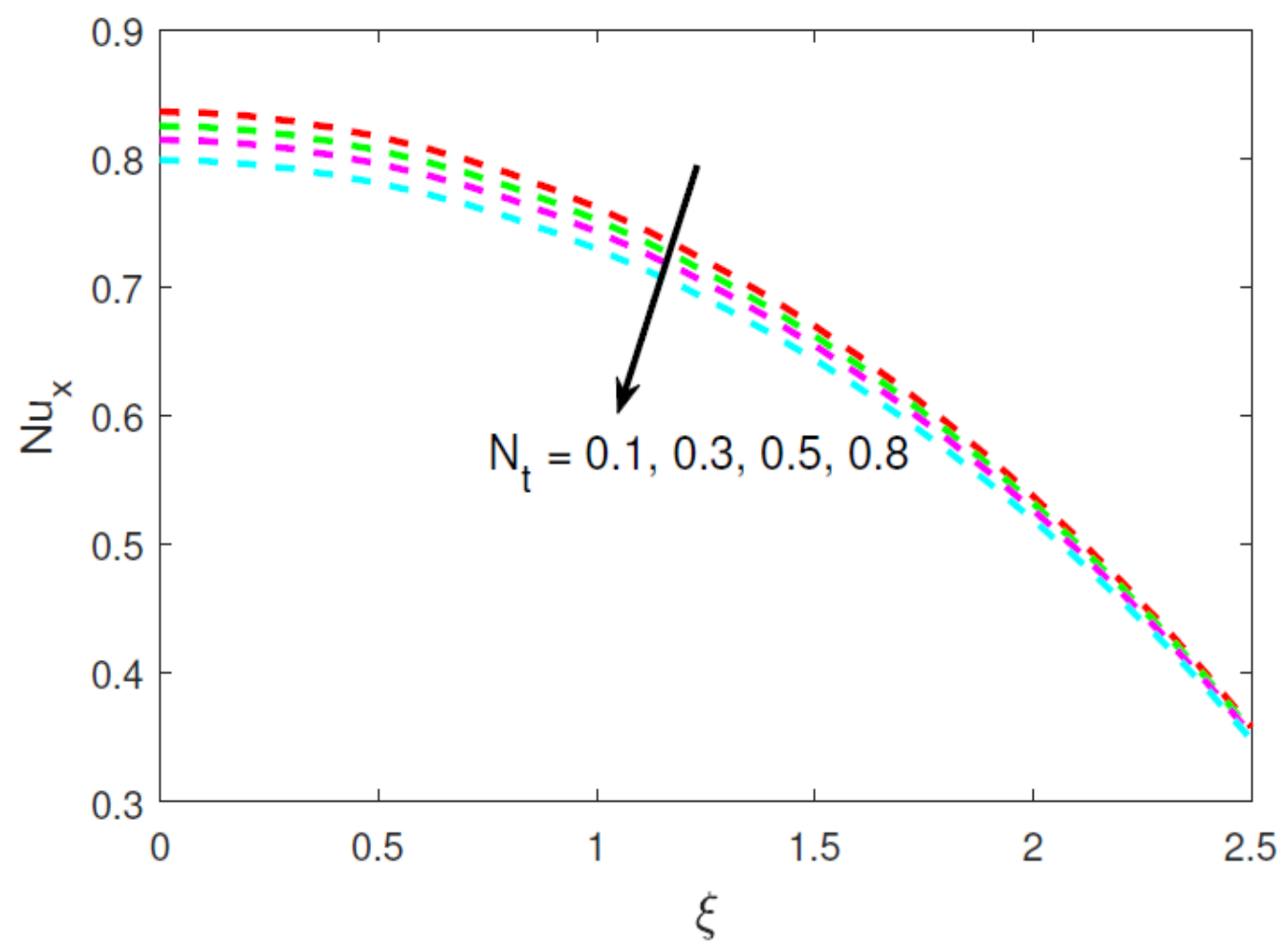

Figure 27. $N u_{x}$ Variations for enhancing $N_{t}$.

clarifies that higher magnetic field parameter values and radiative heat flux values decline the skin friction factor. The skin friction factor is strongly augmented with increasing stream wise coordinate $(\xi)$ up to a critical point at which it peaks $\xi \sim 2$ there after descending steadily with further stream wise distance, as characterized by the parabolictype topology. In addition, it is perceived that influence of $H a$ on $C f_{x}$ is more dominant than the influence of $R$. Figure 23 indicates that the skin friction factor $\left(C f_{x}\right)$ minutely increases for amplifying thermal conductivity variation parameter. In other words, a weak acceleration is seen in the skin friction profiles with higher thermal conductivity of the nanofluid. Momentum diffusion must therefore be augmented with increasing ability of the nanofluid to transport heat. This trend has been reported earlier by Takhar and Beg [55] albeit for non-magnetic boundary layer flows.

Figures 24 and 25 present the evolution in Nusselt number $\left(N u_{x}\right)$ for variation in magnetic field parameter $(\mathrm{Ha})$ and thermal radiation parameter $(R)$, respectively. Magnification in magnetic field parameter and thermal radiation parameter values decrease the heat transfer rate profiles. It is noticed that impact of radiative heat flux is more dominant on the Nusselt number distribution at the cylinder surface (wall) compared with the impact of magnetic field parameter. Both magnetic field and radiative flux may therefore be implemented potently to manipulate the heat transmission from the coating to the substrate (cylinder) in materials processing operations. Figure 26 shows that the Nusselt number $\left(N u_{x}\right)$ upsurges for intensifying 


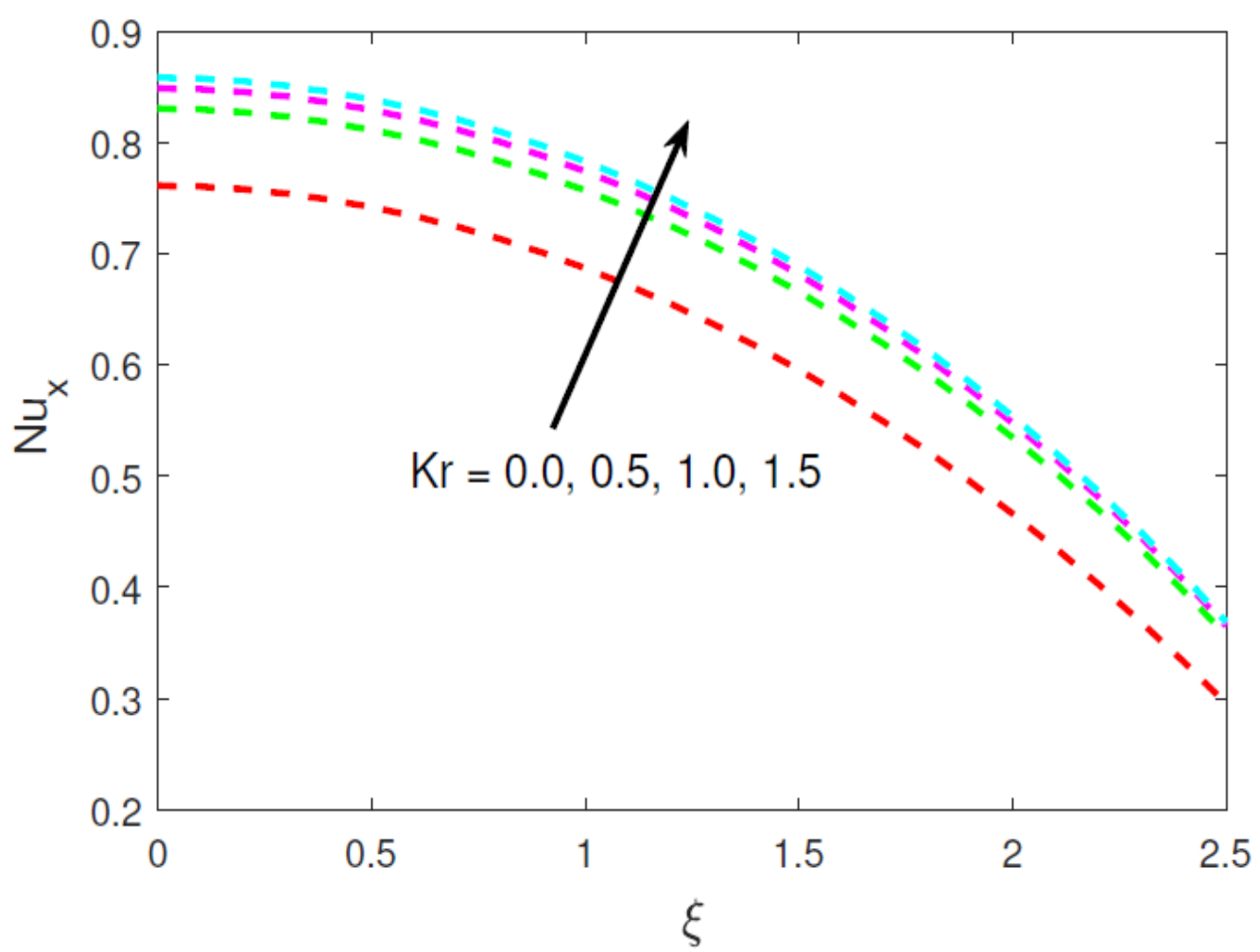

Figure 28. $N u_{x}$ variations for enhancing $K r$

thermal conductivity variation parameter values. Nusselt number is higher near surface of the cylinder $(\xi=0)$ while it uniformly declines for higher stream wise coordinate values (away from the surface). The results reveal that heat transfer to the cylindrical surface can be computed with greater physical accuracy in the presence of variable thermal conductivity. Figure 27 shows that Nusselt number $\left(N u_{x}\right)$ is suppressed with higher nanoscale thermophoresis parameter $\left(N_{t}\right)$ values i.e. Nusselt number at the wall is a declining function of thermophoresis. This corroborates the earlier computations which have shown that temperature is elevated with stronger thermophoretic body force. Since the Powell-Eyring nanofluid is heated with this effect, there is an associated decrement in fluid temperature to the cylindrical surface (boundary) i.e. lower Nusselt numbers. Figure 28 implies that magnitudes of Nusselt number $\left(N u_{x}\right)$ intensify for larger chemical reaction parameter $(K r)$ values. Hence the temperature near the wall is assisted with stronger homogenous destructive chemical reaction of the nanoparticles.

As seen in Fig. 29, Sherwood number $\left(S h_{x}\right)$ profiles diminish for magnifying the thermophoresis parameter. It is apparent from Fig. 30 that Sherwood number $\left(S h_{x}\right)$ profiles are enhanced with higher chemical reaction parameter $(K r)$ values which promotes migration of nanoparticles to the cylinder surface and diminishes mass transfer profiles.

Table 4 shows the numerical values of local skin friction factor $\left(C f_{x}\right)$, local Nusselt 


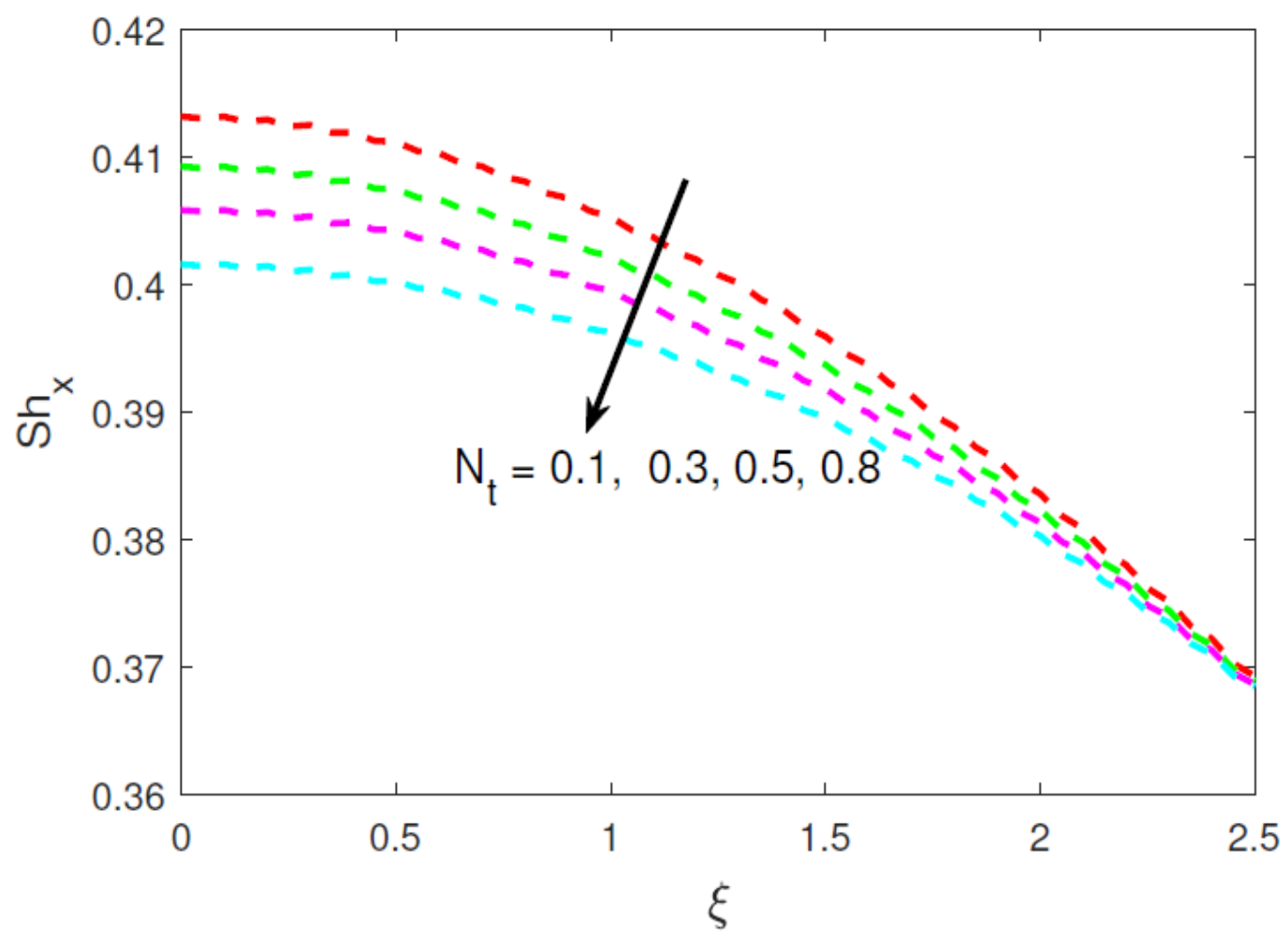

Figure 29. $S h_{x}$ variations for enhancing $N_{t}$

number $\left(N u_{x}\right)$ and local Sherwood number $\left(S h_{x}\right)$ for Newtonian $\left(\varepsilon=\varepsilon_{1}=0\right)$ and nonNewtonian $\left(\varepsilon\right.$ and $\left.\varepsilon_{1}>0\right)$ cases. It is observed that the skin friction coefficient enhances for intensified Darcy number values, thermal conductivity parameter, Brownian parameter, chemical reaction and Schmidt number for both Newtonian and nonNewtonian cases. The skin friction factor declines with amplifying radiative heat flux values. It is apparent that skin friction values slightly increase for increasing thermophoresis parameter in the case of Newtonian fluid whereas skin friction value remains constant for the given values of thermophoresis parameter in case of nonNewtonian fluid. Nusselt number and Sherwood number are clearly boosted with higher thermal conductivity parameter values, Schmidt number and chemical reaction parameter whereas they are depressed for magnifying thermal radiation parameter and thermophoresis parameter. Higher Brownian motionparameter values declines the Nusselt number while the reverse trend is perceived in Sherwood number distribution.

\section{Conclusions}

Motivated by simulating the transport phenomena characteristics of magnetic nanofluid coatings on engineering component substrates, a detailed mathematical study has been presented to investigate the thermo solutal (combined natural convection heat and mass transfer) characteristics in magnetohydrodynamic, radiative, reactive nanofluid 


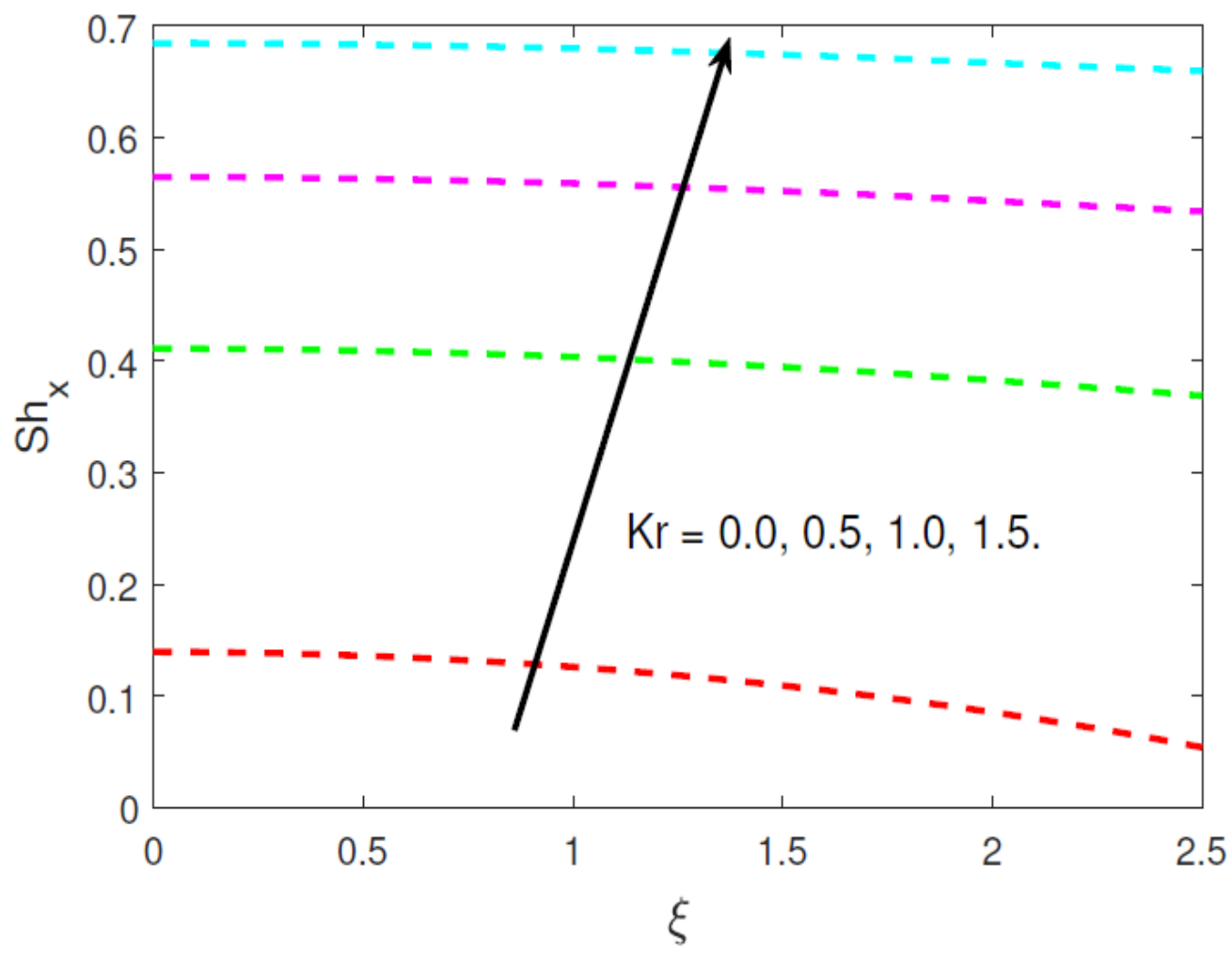

Figure 30. $S h_{x}$ variations for enhancing $K r$

boundary layer flow external to a circular cylinder in a non-Darcy porous medium with variable thermal conductivity. The nanoscale transport is modelled by employing the Buongiorno two-component model and the rheological behaviour is accommodated with the Powell-Eyring model. The resulting nonlinear coupled boundary value problem is solved with the implicit finite difference KBM scheme under appropriate wall and free stream boundary conditions. Extensive validation of present results with published research articles is included. The heat, mass and momentum characteristics are studied for various thermophysical parameters, visualized through graphs and summarized in tables. The main findings are as follows:

- Fluid velocity decreases with larger values of the second Powell-Eyring rheological parameter and magnetic field parameter.

- Nanoparticle concentration declines with larger value of first order homogenous destructive chemical reaction parameter, Brownian motion parameter and Schmidt number.

- The influence of the Schmidt number and chemical reaction parameter on Nusselt number and Sherwood number are opposite to those induced by the thermophoresis parameter and thermal radiation for both Newtonian and non-Newtonian cases.

- Heat transfer rate (Nusselt number) at transverse coordinate increases with 
Table 4. Variations in $C f_{x}, N u_{x}$ and $S h_{x}$ at $\xi=1$ for Newtonian and non-Newtonian fluids.

\begin{tabular}{|c|c|c|c|c|c|c|c|}
\hline & & \multicolumn{3}{|c|}{ Newtonian fluid $\varepsilon=\varepsilon_{1}=0$} & \multicolumn{3}{|c|}{ non- Newtonian fluid $\varepsilon=\varepsilon_{1}=0.2$} \\
\hline Parameter & Values & $C f_{x}$ & $N u_{x}$ & $S h_{x}$ & $C f_{x}$ & $N u_{x}$ & $S h_{x}$ \\
\hline \multirow{3}{*}{$\wedge$} & 0.1 & 0.2566 & 0.9997 & 0.5171 & 0.0769 & 0.4840 & 0.5097 \\
\hline & 0.3 & 0.2557 & 0.9989 & 0.5170 & 0.0767 & 0.4836 & 0.5096 \\
\hline & 0.5 & 0.2549 & 0.9982 & 0.5169 & 0.0765 & 0.4832 & 0.5093 \\
\hline \multirow{3}{*}{$D a$} & 0.1 & 0.1310 & 0.9115 & 0.5062 & 0.0254 & 0.4237 & 0.5048 \\
\hline & 0.3 & 0.2123 & 0.9623 & 0.5126 & 0.0568 & 0.4668 & 0.5066 \\
\hline & 0.5 & 0.2549 & 0.9982 & 0.5169 & 0.0765 & 0.4832 & 0.5097 \\
\hline \multirow{3}{*}{$R$} & 0.1 & 0.2562 & 1.7935 & 0.5174 & 0.2478 & 1.7858 & 0.4004 \\
\hline & 0.3 & 0.2537 & 0.7329 & 0.5165 & 0.2455 & 0.7255 & 0.3989 \\
\hline & 0.5 & 0.2519 & 0.5190 & 0.5158 & 0.2438 & 0.5120 & 0.3978 \\
\hline \multirow{3}{*}{$\gamma^{*}$} & 0.1 & 0.2547 & 0.9210 & 0.5167 & 0.2464 & 0.9140 & 0.3993 \\
\hline & 0.2 & 0.2549 & 0.9982 & 0.5169 & 0.2466 & 0.9906 & 0.3996 \\
\hline & 0.3 & 0.2550 & 1.0745 & 0.5171 & 0.2468 & 1.0664 & 0.3998 \\
\hline \multirow{3}{*}{$N_{t}$} & 0.1 & 0.2548 & 1.0026 & 0.5173 & 0.2466 & 0.9949 & 0.4002 \\
\hline & 0.2 & 0.2549 & 0.9982 & 0.5169 & 0.2466 & 0.9906 & 0.3996 \\
\hline & 0.3 & 0.2552 & 0.9938 & 0.5167 & 0.2466 & 0.9863 & 0.3991 \\
\hline \multirow{3}{*}{$N_{b}$} & 0.1 & 0.2541 & 1.0119 & 0.5132 & 0.2456 & 1.0032 & 0.3946 \\
\hline & 0.2 & 0.2546 & 1.0052 & 0.5160 & 0.2463 & 0.9971 & 0.3983 \\
\hline & 0.3 & 0.2549 & 0.9982 & 0.5169 & 0.2466 & 0.9906 & 0.3996 \\
\hline \multirow{3}{*}{$K r$} & 0.5 & 0.2549 & 0.9982 & 0.5169 & 0.2438 & 0.5120 & 0.3978 \\
\hline & 1.0 & 0.2685 & 1.0064 & 0.7183 & 0.2557 & 0.5203 & 0.5557 \\
\hline & 1.5 & 0.2777 & 1.0107 & 0.8748 & 0.2640 & 0.5248 & 0.6777 \\
\hline \multirow{3}{*}{$S c$} & 0.1 & 0.2302 & 0.9775 & 0.2327 & 0.2288 & 0.4987 & 0.2298 \\
\hline & 0.3 & 0.2454 & 0.9912 & 0.3997 & 0.2438 & 0.5120 & 0.3978 \\
\hline & 0.5 & 0.2549 & 0.9982 & 0.5169 & 0.2532 & 0.5186 & 0.5156 \\
\hline
\end{tabular}

boosting thermal conductivity parameter values and porous media permeability parameter (Darcy number).

- Velocity magnitude is elevated with higher values of the first Powell-Eyring rheological parameter whereas velocity magnitude is decreased for higher Darcy number values (i.e. progressively extra permeable regimes).

- Fluid temperature enhances for higher thermal conductivity variation parameter and magnetic field parameter values; however the contrary pattern is computed with greater thermal radiation parameter.

The present study has considered steady state magnetohydrodynamic nanofluid coating flow with homogenous chemical reaction. Future investigations may address unsteady hydromagnetic flows (Beg et al. [56]) and also consider heterogenous chemical reactions (Mishra et al. [57]), which are also of interest in smart coating transport phenomena. 


\section{References}

[1] R. E. Powell, H. Eyring, Mechanisms for the relaxation theory of viscosity. Nature 154 (1944) 4278.

[2] T. Hayat, M. Ijaz Khan, M. Waqas, A. Alsaedi, Effectiveness of magnetic nanoparticles in radiative flow of Eyring-Powell fluid, J. Mol. Liq. 231 (2017) 126133.

[3] K. Rehman, M.Y. Malik, S. Bilal, M. Bibi, U. Ali, Logarithmic and parabolic curve fitting analysis of dual stratified stagnation point MHD mixed convection flow of Eyring-Powell fluid induced by an inclined cylindrical stretching surface. Results Phys. 7 (2017) 544552.

[4] S. S. Ghadikolaei, K. Hosseinzadeh, D.D. Ganji, Analysis of unsteady MHD Eyring-Powell squeezing flow in stretching channel with considering thermal radiation and Joule heating effect using AGM, Case Studies Therm. Eng. 10 (2017) 579594.

[5] N. Balazadeh, M. Sheikholeslami, D.D. Ganji, Zhixiong. Li, Semi-analytical analysis for transient Eyring-Powell squeezing flow in a stretching channel due to magnetic field using DTM, J. Mol. Liq. 260 (2018) 3036.

[6] T. Hayat, J. Akram, A. Alsaedi, H. Zahir, Endoscopy effect in mixed convective peristalsis of Powell-Eyring nanofluid, J. Mol. Liq. 254 (2018) 4754.

[7] S.O. Salawu, A.R. Hassan, A. Abolarinwa, N.K. Oladejo, Thermal stability and entropy generation of unsteady reactive hydromagnetic Powell-Eyring fluid with variable electrical and thermal conductivities, Alexandria Eng. J. (2019) 58, 519529.

[8] M. A. Seddeek, S.N. Odda, M.S. Abdelmeguid, Numerical study for the effects of thermophoresis and variable thermal conductivity on heat and mass transfer over an accelerating surface with heat source, Comput. Mater. Sci. 47 (2009) 9398.

[9] M. Khan, R. Malik, Forced convective heat transfer to Sisko nanofluid past a stretching cylinder in the presence of variable thermal conductivity, J. Mol. Liq.218 (2016) 17.

[10] R. Sivaraj, A. Jasmine Benazir, S. Srinivas, and A.J. Chamkha, Investigation of cross-diffusion effects on Casson fluid flow in existence of variable fluid properties, Eur. Phys. J. Spec. Top.228 (2019) 3553.

[11] M. Madhu, N. S. Shashikumar, B. J. Gireesha and Naikoti Kishan Second law analysis of PowellEyring fluid flow through an inclined microchannel with thermal radiation, Physica Scripta, 94(2019).

[12] S. Chaudhary and K. M. Kanika, Radiation heat transfer on SWCNT and MWCNT based magnetohydrodynamic nanofluid flow with marangoni convection Physica Scripta, 95(2) (2019).

[13] I. Ullah, Tasawar Hayat, Ahmed Alsaedi and Saleem Asghar, Dissipative flow of hybrid nanoliquid (H2O-aluminum alloy nanoparticles) with thermal radiation Physica Scripta,94 (12) (2019).

[14] D. Mythili, R. Sivaraj, Influence of higher order chemical reaction and non-uniform heat source/sink on Casson fluid flow over a vertical cone and flat plate, J. Mol. Liq.216 (2016) 466475 .

[15] Z. Abbas, M. Sheikh, S.S. Motsa, Numerical solution of binary chemical reaction on stagnation point flow of Casson fluid over a stretching / shrinking sheet with thermal radiation, Energy 95 (2016) $12 \mathrm{e} 20$.

[16] M. Rahman, M. Manzur, M. Khan, Mixed convection heat transfer to modified second grade fluid in the presence of thermal radiation, J. Mol. Liq. 223 (2016) 217223.

[17] M. Sheikholeslami, Hari R.Kataria, AkhilS.Mittal, Radiation effects on heat transfer of three dimensional nanofluid flow considering thermal interfacial resistance and micro mixing in suspensions, Chin. J. Phys. 55,(6), (2017) 2254-2272.

[18] M. Khan, HumaraSardar, Hashim,Heat generation/absorption and thermal radiation impacts on three-dimensional flow of Carreau fluid with convective heat transfer, J. Mol. Liq. 272 (2018) 474480 .

[19] N. Sandeep, B. Rushi Kumar, M.S. Jagadeesh Kumar, A comparative study of convective heat and mass transfer in non-Newtonian nanofluid flow past a permeable stretching sheet, J. Mol. 
Liq. 212 (2015) 585591.

[20] O.D. Makinde, I.L. Animasaun, Thermophoresis and Brownian motion effects on MHD bioconvection of nanofluid with nonlinear thermal radiation and quartic chemical reaction past an upper horizontal surface of a paraboloid of revolution.J. Mol. Liq.221 (2016) 733743.

[21] A. Hamid, Hashim, Masood Khan, Impacts of binary chemical reaction with activation energy on unsteady flow of magneto-Williamson nano fluid, J. Mol. Liq. 262 (2018) 435442.

[22] A. S. Mittal, H. R. Patel, Influence of thermophoresis and Brownian motion on mixed convection two dimensional MHD Casson fluid flow with non-linear radiation and heat generation, Physica A $537(2020) 122710$.

[23] A. Hussain, Anwar Ullah, Boundary layer flow of a Walters B fluid due to a stretching cylinder with temperature dependent viscosity, Alexandria Eng. J., 55 (2016) 3073-3080.

[24] M. Tamoor, MHD convective boundary layer slip flow and heat transfer over nonlinearly stretching cylinder embedded in a thermally stratified medium, Results Phys., 7(2017)4247-4252.

[25] M. Patel, M.G. Timol, Numerical treatment of Powell-Eyringfluid flow using method of satisfaction of asymptotic boundary conditions (MSABC), Appl. Numer. Math., 59 (2009) 25842592.

[26] T. Hayat, M. Awais, S. Asghar, Radiative effects in a three-dimensional flow of MHD Eyring-Powell fluid, J. Egypt Math.Soc. 21 (2013) 379384.

[27] S. Abdul Gaffar, V. Ramachandra Prasad, E. Keshava Reddy, O. Anwar Bg, Thermal radiation and heat generation/absorption effects on visco-elastic double-diffusive convection from an isothermal sphere in porous media, Ain Shams Eng. J., 6 (2015) 10091030.

[28] T. Hayat, I. Ullah, A. Alsaedi, M. Farooq, MHD flow of Powell-Eyring nanofluid over a non-linear stretching sheet with variable thickness, Results Phys. 7 (2017) 189196.

[29] A. Raptis, Radiation and viscoelastic flow, Int. Commun. Heat Mass Transfer 26 (1999) 889895.

[30] S. Reddy, Kishan Naikoti, Mohammad Mehdi Rashidi, MHD flow and heat transfer characteristics of Williamson nanofluid over a stretching sheet with variable thickness and variable thermal conductivity,Transactions of A. Razmadze Mathematical Institute,171 (2) (2017) 195-211.

[31] T. Cebeci, and P. Bradshaw, Physical and Computational Aspects of Convective Heat Transfer, Springer, Berlin (1984).

[32] J.H. Merkin, Free convection boundary layers on cylinders of elliptic cross section, ASME J. Heat Transfer, 99 (1977) 453-457.

[33] A. Guiseppi-Elie,Electroconductive hydrogels: synthesis, characterization and biomedical applications, Biomaterials, 31 (2010) 2701-2716.

[34] J. Prakash, E.P. Siva, D Tripathi, S. Kuharatand O. Anwar Bg, Peristaltic pumping of magnetic nanofluids with thermal radiation and temperature-dependent viscosity effects: modelling a solar magneto-biomimetic nanopump, Renewable Energy, 133 (2019) 1308-1326.

[35] R. B. Mansour, and R. Viskanta, Radiative and convective heat transfer for materials processing, in Proceedings of the First Conference on Transport Phenomena in Processing, S.I. Guceri, Editor, (1992) 693713, Technomic Publishing Co., Lancaster, PA, USA.

[36] Hughes, F. William. and Young, J. Frederick, The Electro-magneto-dynamics of Fluids, John Wiley, New York, USA (1966).

[37] J. C. Umavathi and $\mathrm{O}$. Anwar $\mathrm{Bg}$, Numerical study of double-diffusive dissipative reactive convective flow in an open vertical duct containing a non-Darcy porous medium with Robin boundary conditions, J. Eng. Math.(2019). https://doi.org/10.1007/ s10665-019-10022-w

[38] Z. Khan ,Haroon Ur Rasheed, Saeed Islam, Sahib Noor, Ilyas Khan, Tariq Abbas, Waris Khan, Asiful H. Seikh, El-Sayed M. Sherif, and Kottakkaran Sooppy Nisar, Heat transfer effect on viscoelastic fluid used as a coating material for wire with variable viscosity, Coatings, 10 (2020) 163-178. https://doi.org/10.3390/coatings10020163

[39] Y.Yu, Guoying Wei, Xiukun Hu, Hongliang Ge, Zhou Yu,The effect of magnetic fields on the electroless deposition of CoWP film, Surface Coatings Tech., 22 (2010) 2669-2676.

[40] R.Ganesan, B.Akhavan, X.Dong, D. R. McKenzie, M.M.M.Bilek, External magnetic field increases both plasma generation and deposition rate in HiPIMS, Surface Coatings Tech., 35 (2018) 671- 
679.

[41] L.Wang, J.Yao, Yong Hu, Qunli Zhang, Zhuo Sun, and Rong Liu., Influence of electric-magnetic compound field on the WC particles distribution in laser melt injection, Surface Coatings Tech., 34 (2017) 32-43.

[42] M. Ishaq, G. Ali, Z. Shah, S. Islam, S. Muhammad, Entropy generation on nanofluid thin film flow of EyringPowell fluid with thermal radiation and MHD effect on an unsteady porous stretching sheet, Entropy, 20 (2018) 412-437.

[43] K. Vafai, and C. L. Tien, Boundary and inertia effects on flow and heat transfer in porous media. Int. J. Heat Mass Transfer 24 (1981) 195203.

[44] Y. Jaluria, and B. Gebhart, Buoyancy-Induced Flow and Transport, Hemisphere, Washington, USA (1988).

[45] M. Modest, Radiative Heat Transfer, MacGraw-Hill, New York, USA (1992).

[46] O. A. Beg, S.K. Ghosh, M. Narahari and T. A. Bg, Mathematical modelling of thermal radiation effects on transient gravity-driven optically-thick gray convection flow along an inclined plate with pressure gradient, Chem. Eng.Commun., 198 (2011) 1-15.

[47] D. Lu, M. Ramzan, Shafiq Ahmad, Ahmad Shafee and Muhammad Suleman, Impact of nonlinear thermal radiation and entropy optimization coatings with hybrid nanoliquid flow past a curved stretched surface, Coatings, 8 (2018) 430-440. https://doi.org/10.3390/coatings 8120430

[48] Y. Jaluria, Thermal issues in materials processing, ASME J Heat Transfer, 135 (2014) 061701-1061701-14.

[49] J. Buongiorno, Convective transport in nanofluids, ASME J Heat Transfer, 128(3) (2006) 240-250.

[50] R. Sivaraj and B. Rushi Kumar, Unsteady MHD dusty viscoelastic fluid Couette flow in an irregular channel with varying mass diffusion, Int. J. Heat Mass Transfer 55 (2012) 30763089.

[51] R. Sivaraj and B. Rushi Kumar, Chemically reacting dusty viscoelastic fluid flow in an irregular channel with convective boundary, Ain Shams Eng. J. 4 (2013) 93101.

[52] B. Rushi Kumar and R. Sivaraj, MHD viscoelastic fluid non-Darcy flow over a vertical cone and a flat plate, Int. Commun. Heat Mass Transfer, 40 (2013) 1-6.

[53] S. R. Sheri, O. Anwar Bg, P. Modugulaand A. Kadir, Computation of transient radiative reactive thermo-solutal magneto-hydrodynamic convection in inclined MHD Hall generator flow with dissipation and cross diffusion, Comput.Therm. Sci., 11 (6) (2019) 541-563.

[54] O. A. Beg, Tasveer A. Beg, I. Karim, M. S. Khan, M. M. Alam, M Ferdows, M. Shamshuddin, Numerical study of magneto-convective heat and mass transfer from inclined surface with Soret diffusion and heat generation effects: A model for ocean magnetohydrodynamic energy generator fluid dynamics, Chin. J. Phys., 60 (2019) 167-179.

[55] H. S. Takhar, and O. Anwar Bg, Non-Darcy convective boundary layer flow past a semi-infinite vertical plate in saturated porous media, Heat Mass Transfer, 32 (11996) 33-44..

[56] O. A. Beg, M. Ferdows; M. Enamul Karim; M. Maruf Hasan; Tasveer A. Bg; MD.Shamshuddin; A. Kadir, Computation of non-isothermal thermo-convective micropolar fluid dynamics in a Hall MHD generator system with non-linear distending wall, Int. J. Appl.Comput.Math., 6 (2020). $1-44$

[57] S. R. Mishra, M. Shamshuddin, , O. Anwar Beg and Ali Kadir, Adomian computation of radiativeconvective bi-directional stretching flow of a magnetic non-Newtonian fluid in a porous medium with homogeneousheterogeneous reactions, Int. J. Modern Phys. B, 33 (2)(2020).

[58] V. R. Prasad, S. Abdul Gaffar, and B. Rushi Kumar,Non-similar computational solutions for double-diffusive MHD transport phenomena for non-Newtonian nanofluid from a horizontal circular cylinder, Nonlinear Engineering, 8 (2019)470485.

[59] H. Thameem Basha, R. Sivaraj, V. Ramachandra Prasad and O. A. Beg,Entropy generation of tangent hyperbolic nanofluid flow over a circular cylinder in the presence of nonlinear Boussinesq approximation: a nonsimilar solution, Journal of thermal Analysis and Calorimetry,(2020). https://doi.org/10.1007/s10973-020-09981-5.

[60] S. Ghosh, S.Sarkar, R. Sivakumar, and T. V. S. Sekhar, Full magnetohydrodynamic flow past a 
circular cylinder considering the penetration of magnetic field, PHYSICS OF FLUIDS 30 (2018) 087102

[61] S. Ghosh, S.Sarkar, R. Sivakumar, and T. V. S. Sekhar, Forced convection magnetohydrodynamic flow past a circular cylinder by considering the penetration of magnetic field inside it, Numerical Heat Transfer, Part A: Applications 76 (1), 32-49 (2019). 